\title{
QUEEN'S
UNIVERSITY
BELFAST
}

\section{A review of high magnetic moment thin films for microscale and nanotechnology applications}

Scheunert, G., Heinonen, O., Hardeman, R., Lapicki, A., Gubbins, M., \& Bowman, R. M. (2016). A review of high magnetic moment thin films for microscale and nanotechnology applications. Applied Physics Reviews, 3, [011301]. https://doi.org/10.1063/1.4941311

Published in:

Applied Physics Reviews

Document Version:

Publisher's PDF, also known as Version of record

Queen's University Belfast - Research Portal:

Link to publication record in Queen's University Belfast Research Portal

Publisher rights

(C) 2016 AIP Publishing. This article may be downloaded for personal use only. Any other use requires prior permission of the author and AIP Publishing.

The following article appeared in Scheunert, G, Heinonen, O, Hardeman, R, Lapicki, A, Gubbins, M \& Bowman, RM 2016, 'A review of high magnetic moment thin films for microscale and nanotechnology applications' Applied Physics Reviews, vol 3, 011301 and may be found at http://scitation.aip.org/content/aip/journal/apr2/3/1/10.1063/1.4941311

\section{General rights}

Copyright for the publications made accessible via the Queen's University Belfast Research Portal is retained by the author(s) and / or other copyright owners and it is a condition of accessing these publications that users recognise and abide by the legal requirements associated with these rights.

Take down policy

The Research Portal is Queen's institutional repository that provides access to Queen's research output. Every effort has been made to ensure that content in the Research Portal does not infringe any person's rights, or applicable UK laws. If you discover content in the Research Portal that you believe breaches copyright or violates any law, please contact openaccess@qub.ac.uk. 


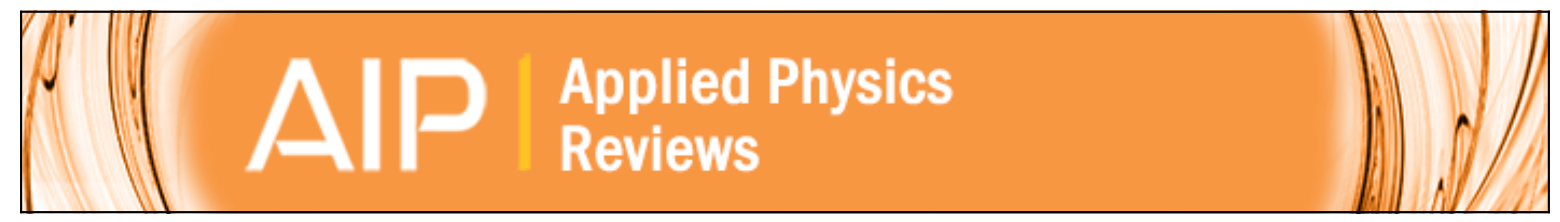

\section{A review of high magnetic moment thin films for microscale and nanotechnology applications \\ G. Scheunert, O. Heinonen, R. Hardeman, A. Lapicki, M. Gubbins, and R. M. Bowman}

Citation: Applied Physics Reviews 3, 011301 (2016); doi: 10.1063/1.4941311

View online: http://dx.doi.org/10.1063/1.4941311

View Table of Contents: http://scitation.aip.org/content/aip/journal/apr2/3/1?ver=pdfcov

Published by the AIP Publishing

\section{Articles you may be interested in}

Application of Landau-Lifshitz-Bloch dynamics to grain switching in heat-assisted magnetic recording

J. Appl. Phys. 112, 013914 (2012); 10.1063/1.4733311

Effect of van der Waals forces on molecularly thin lubricant in the magnetic storage head-disk interface J. Appl. Phys. 98, 124906 (2005); 10.1063/1.2148633

Modeling of thermal magnetization fluctuations in thin-film magnetic devices

J. Appl. Phys. 90, 5768 (2001); 10.1063/1.1402146

Head parameter sensitivity study of the intrinsic field reversal time

J. Appl. Phys. 85, 4979 (1999); 10.1063/1.370064

Magnetic properties of FeAIN films at elevated temperatures

J. Appl. Phys. 81, 4495 (1997); 10.1063/1.364989

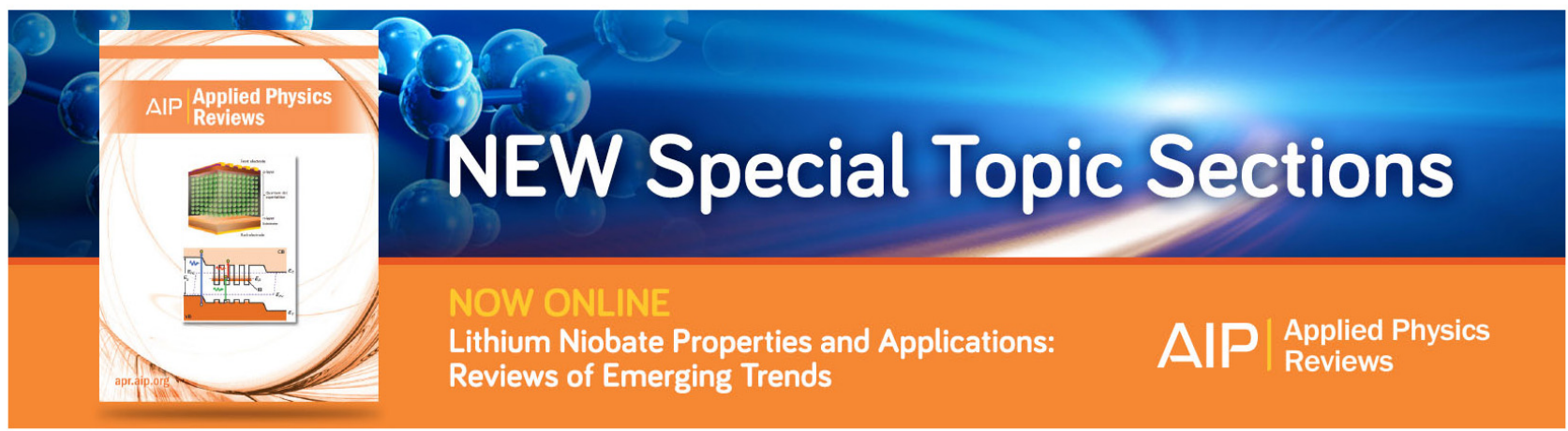




\title{
A review of high magnetic moment thin films for microscale and nanotechnology applications
}

\author{
G. Scheunert, ${ }^{1,2, a)}$ O. Heinonen, ${ }^{3,4}$ R. Hardeman, ${ }^{5}$ A. Lapicki, ${ }^{5}$ M. Gubbins, ${ }^{5}$ \\ and R. M. Bowman ${ }^{2}$ \\ ${ }^{1}$ Department of Physics of Complex Systems, Weizmann Institute of Science, 76100 Rehovot, Israel \\ ${ }^{2}$ Centre for Nanostructured Media, School of Mathematics and Physics, Queen's University of Belfast, \\ Belfast BT7 1NN, United Kingdom \\ ${ }^{3}$ Materials Science Division, Argonne National Laboratory, 9700 South Cass Ave., Lemont, Illinois 60439, \\ USA \\ ${ }^{4}$ Northwestern-Argonne Institute of Science and Technology, Evanston, Illinois 60208, USA \\ ${ }^{5}$ Seagate Technology (Ireland), Springtown Industrial Estate, 1 Disc Drive, Derry BT48 OBF, United Kingdom
}

(Received 13 September 2015; accepted 1 December 2015; published online 17 February 2016)

\begin{abstract}
The creation of large magnetic fields is a necessary component in many technologies, ranging from magnetic resonance imaging, electric motors and generators, and magnetic hard disk drives in information storage. This is typically done by inserting a ferromagnetic pole piece with a large magnetisation density $M_{\mathrm{S}}$ in a solenoid. In addition to large $M_{\mathrm{S}}$, it is usually required or desired that the ferromagnet is magnetically soft and has a Curie temperature well above the operating temperature of the device. A variety of ferromagnetic materials are currently in use, ranging from FeCo alloys in, for example, hard disk drives, to rare earth metals operating at cryogenic temperatures in superconducting solenoids. These latter can exceed the limit on $M_{\mathrm{S}}$ for transition metal alloys given by the Slater-Pauling curve. This article reviews different materials and concepts in use or proposed for technological applications that require a large $M_{\mathrm{S}}$, with an emphasis on nanoscale material systems, such as thin and ultra-thin films. Attention is also paid to other requirements or properties, such as the Curie temperature and magnetic softness. In a final summary, we evaluate the actual applicability of the discussed materials for use as pole tips in electromagnets, in particular, in nanoscale magnetic hard disk drive read-write heads; the technological advancement of the latter has been a very strong driving force in the development of the field of nanomagnetism. (C) 2016 AIP Publishing LLC. [http://dx.doi.org/10.1063/1.4941311]
\end{abstract}

\section{TABLE OF CONTENTS}

I. INTRODUCTION

A. Magnetic moment and saturation

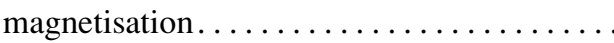

B. A brief introduction to first-principle based computational techniques: Density

Functional Theory (DFT) .............

C. Stoner criterion $\ldots \ldots \ldots \ldots \ldots \ldots \ldots \ldots$

D. High moment materials: The benchmark case of the HDD write pole tip .........

E. Fabrication techniques suitable for the creation of magnetic (ultra-) thin films and nanostructures....................

1. Electroplating and electroless deposition.

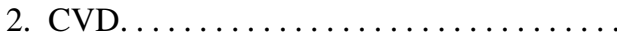

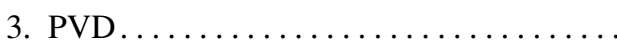

4. Seed layers and strain engineering ......

5. Concluding remarks

\footnotetext{
${ }^{a)}$ Electronic mail: gunther.scheunert@weizmann.ac.il
}

F. Magnetometry: Measuring the magnetic moment and magnetisation.............

1. Magnetometry techniques............

2. On the limits of magnetometry ........

G. Cooling solid nanostructures ............

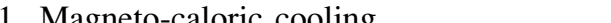

2. Giant Peltier effect ................. 11

3. Field-emission or thermionic cooling.... 12

4. Optical refrigeration: Laser cooling of solids ................... 12

5. Summary: Cooling nanostructures ..... 12

II. REVIEW OF HIGH-MOMENT MAGNETIC MATERIALS ...................... 13

A. Inter-TM alloys of $\mathrm{Fe}, \mathrm{Ni}$, and $\mathrm{Co}$ : The FeCo champion ............... 13

B. TM/NM interfaces, superlattices, and TMNM alloys...................... 15

1. $\mathrm{Fe}$ atoms at $\mathrm{NM}(\mathrm{NM}=\mathrm{Au}, \mathrm{Ag}, \mathrm{Pd})$ interfaces and in nanolaminates .......

2. Fe-NM $(\mathrm{NM}=\mathrm{Au}, \mathrm{Ag}, \mathrm{Pd}$, and $\mathrm{Rh})$ and

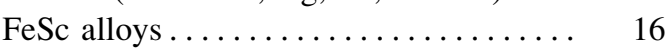

3. FeRh .................... 17 
4. $\mathrm{FeCo} / \mathrm{Pd}$ laminates and $\mathrm{FeCo}: \mathrm{Pd}$ alloys . .

5. FeCo epitaxially grown ultrathin films ..

6. Summary: TM/NM interfaces, superlattices, and TM-NM alloys ......

C. III-V-SC/TM interfaces . . . . . . . . . . .

D. $\mathrm{MnFe} \ldots \ldots \ldots \ldots \ldots \ldots \ldots \ldots \ldots \ldots$

E. FeN $\ldots \ldots \ldots \ldots \ldots \ldots \ldots \ldots \ldots \ldots$

F. Heavy RE metals: Pure elements,

superlattices, and alloys ..............

1. Pure heavy RE metals: Ho, Dy, Tb, and $\mathrm{Gd} \ldots \ldots \ldots \ldots \ldots \ldots \ldots \ldots \ldots \ldots \ldots$

2. Inter-RE alloys and laminates $\ldots \ldots \ldots$.

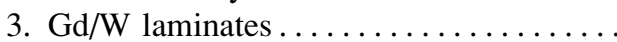

4. Gd-implanted GaN ................

5. Summary: Heavy RE metals, alloys, and compounds ...................

G. Combined RE-TM systems ..........

1. RE-TM/RE-NM alloys and direct

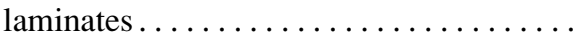

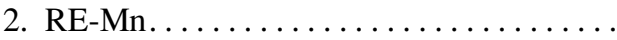

3. RE/IE/TM and RE/Cr/TM systems......

4. Summary: Combined RE-TM systems...

H. A remark on TM and RE clusters and

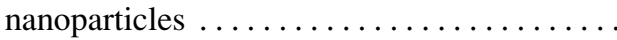

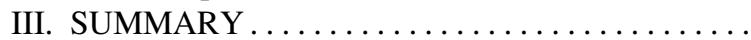

IV. OUTLOOK $\ldots \ldots \ldots \ldots \ldots \ldots \ldots \ldots \ldots \ldots$

19

19

19

20

20

23

24

24

26

26

26

27

27

31

\section{INTRODUCTION}

Magnetism is a particularly striking physical phenomenon and has fascinated humanity since its discovery. Its utility in applications has impacted and been beneficial to, arguably, all facets of our society to date. For the overwhelming period of time that we have used magnetic phenomena, the implementation has been macroscopic, at least down to bulk engineering dimensions. However, the last two decades have seen the emergence of its implementation and the use in a whole host of sub-microscale concepts, devices, and applications. The most striking, for its impact upon our society and the information age, is in data storage. The overwhelming means of local and increasingly remote storage of digital information is through the hard disk drive (HDD). A HDD uses magnetism in the writing, the storage, and retrieval of the binary digital information sent to it. In 2014, some $564 \times 10^{6}$ HDDs were supplied and provided 540 Exabytes of storage capacity. ${ }^{5}$ The growth in areal density, the term used to define the quantity of information encoded, has followed its own "Moore's Law" for many years. By 2015, this has resulted in some astonishing physics, materials, and engineering achievements: a "bit" of information is comprised of less than $50 \mathrm{sub}-10 \mathrm{~nm}$ diameter grains of magnetic media, the "write pole" that encodes the written bit uses material close to the Slater-Pauling limit for magnetisation while having critical dimensions less than $40 \mathrm{~nm}$ and being uniquely shaped in three dimensions and the "reader" uses a complicated multilayer array of normal metals, oxides, ferromagnetic (FM), antiferromagnetic (AFM) coupling, and permanent magnets to exploit quantum effects in tunnelling magnetoresistance in an element around $30 \mathrm{~nm}$ thick and $30 \mathrm{~nm}$ wide. The writer and reader are integrated into a single component, the read-write head, which in totality operates in the upper $\mathrm{MHz}$ frequency regime while "flying" a few nanometres above the recording disk surface whilst travelling at $\approx 50 \mathrm{~m} \mathrm{~s}^{-1}$.

Driven by the current slowdown of the annual increase in storage capacity of HDDs, the storage community is forced to turn to entirely new concepts to boost areal density. ${ }^{6}$ Besides technically very difficult and expensive bit-patterned media (BPM), heat-assisted magnetic recording (HAMR) is believed to be the most promising candidate for the next generation of HDDs. HAMR was proposed as early as 2001 (Refs. 7 and 8) and under serious consideration shortly thereafter. ${ }^{9-11}$ Although it was first demonstrated in a prototype by Seagate Technology already in $2008,{ }^{12}$ initial predictions on the availability of a commercial product in the beginning of this decade as depicted in Fig. 1 have not come true. In fact, recent predictions foretell an introduction of

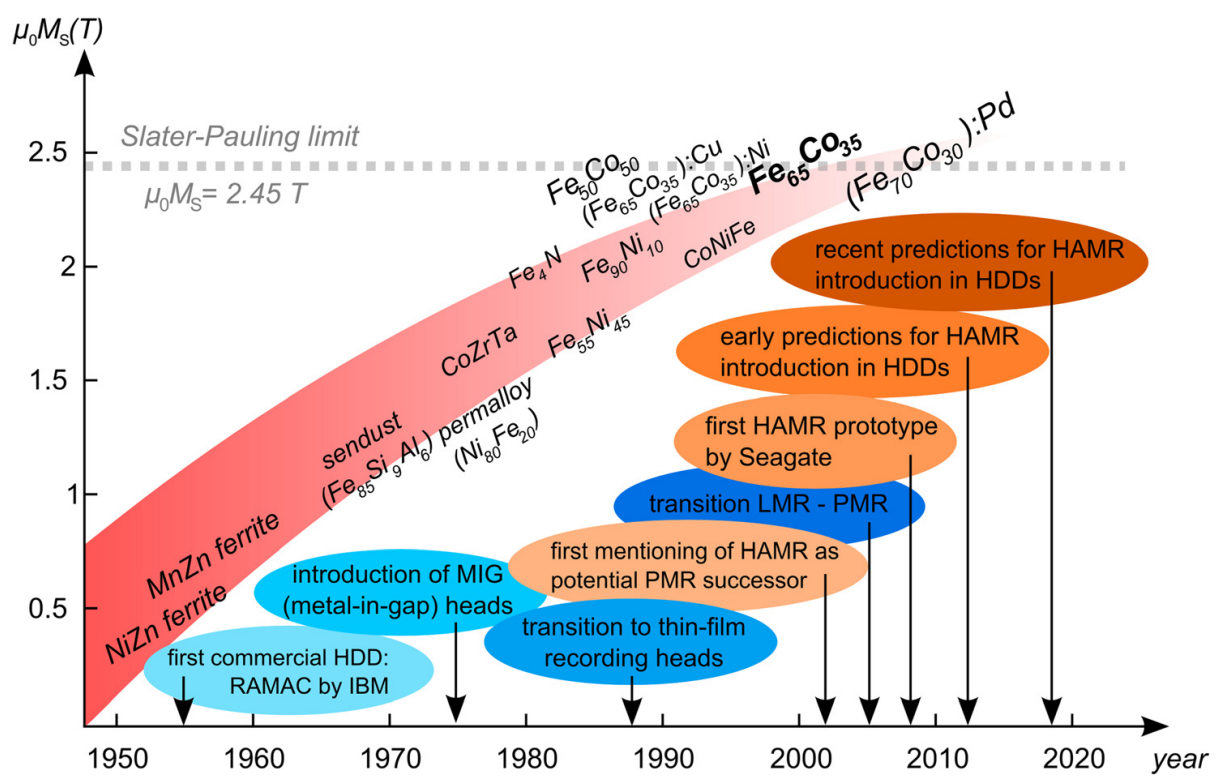

FIG. 1. Development of the HDD pole piece material with high saturation magnetisation over time. The SlaterPauling limit gives the upper known saturation magnetisation $M_{\mathrm{S}}$ of any bulk magnetic material at room temperature, known to date, with $\mu_{0} M_{\mathrm{S}}=2.45 \mathrm{~T}$ for $\mathrm{Fe}_{65-70} \mathrm{CO}_{35-30}$ binary alloys. Although HAMR could be successful in circumventing the need for higher magnetisations in pole tips, technical difficulties have delayed its introduction in commercial HDDs. The $M_{\mathrm{S}}$ data is based on reviews by Buschow and Boer, ${ }^{1}$ Kryder, ${ }^{2}$ Cooper et al., ${ }^{3}$ and Noma et al. ${ }^{4}$ 
commercial HDDs featuring HAMR not earlier than $2017 .{ }^{13}$ The main obstacle for successful operation is an issue with the reliability of these laser-driven drives. ${ }^{14}$ The light delivery poses the most serious challenge because focussing the heat to the required nanometre spot size by means of a nearfield transducer featuring a plasmonic resonator ${ }^{15}$ inevitably leads to high losses. ${ }^{16}$ Those in turn cause material degradation (particularly of the lubricant on the surface of the recording medium ${ }^{17}$ ) and eventually result in total device failure. $^{18}$

The last major breakthrough in HDD technology was the transition from longitudinal magnetic recording (LMR) to perpendicular magnetic recording (PMR), ${ }^{19}$ as depicted in Figs. 2(a) and 2(b), respectively. The perpendicular orientation of the magnetic grains in the recording medium allowed smaller bit feature size, at an increased particle volume which improved thermal stability. With fundamental changes in the device design, such as the introduction of the singlepole write head, the write pole moved closer to the recording medium and, rather than using the fringing field of a dualpole write head as in LMR, the direct stray field in the air gap of this nanodimensional electromagnet could now be used for switching the magnetic bits. Both did significantly boost the available write-field strength $^{20}$ and therefore enabled the use of even harder CoCrPt recording medium, consisting of smaller grains of higher coercivity, eventually leading to an increased areal density. ${ }^{21}$

However, conventional PMR has nearly reached its limit and present day predictions do not go far beyond an areal

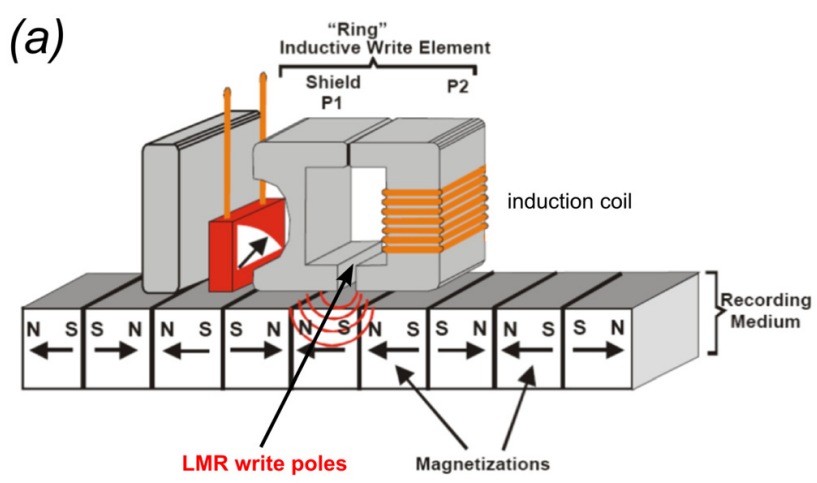

(b)

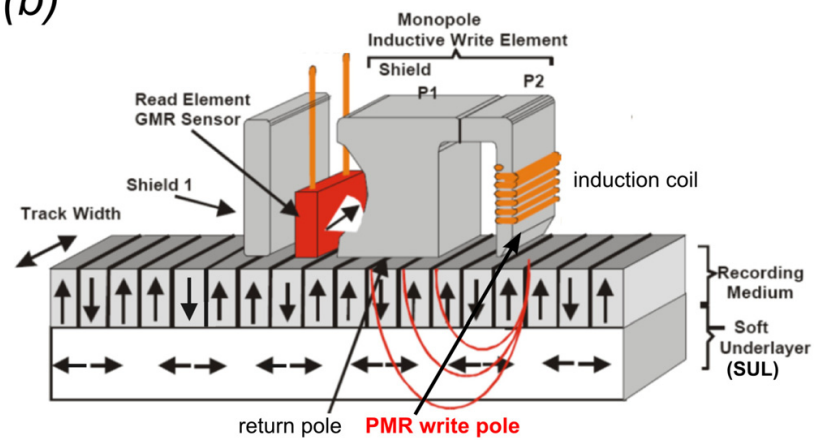

FIG. 2. Operating principles of (a) longitudinal magnetic recording (LMR) and (b) perpendicular magnetic recording (PMR). Graphs (a) and (b) are reproduced with permission from Wood et al., White Paper. Copyright 2007 Hitachi Global Storage Technology. ${ }^{28}$ density of $1 \mathrm{~T}$ bit per square-inch. ${ }^{22,23}$ What appears to be a fundamental limitation is best described by the "trilemma", where the three main contributors to areal density are in competition: write-ability, read-ability, and thermal stability. ${ }^{24}$ Addressing one of the three leads to the problems with the other two. For instance, to assure long-term thermal stability, the recording medium needs to have high coercivity and decent saturation magnetization (i.e., squareness). This is particularly important since the read-ability requires the media to consist of smallest possible grains (for sharper bit transitions and a better signal-to-noise ratio), which would suffer from superparamagnetism (SPM) if the recording medium would not have high enough coercivity. This highanisotropy recording material however would require a very tightly focused write field of sufficiently large strength to overcome the media coercivity for flipping the magnetisation of the grains. But the write field strength is limited by the geometry of the head-media ensemble and ultimately by the write pole tip's saturation magnetisation. ${ }^{20}$

Recently introduced shingled recording, where adjacent recording tracks on the media platter are overlayed, eases the geometrical requirements of the write pole as its tip surface area can be bigger than the actual bit feature size. ${ }^{25}$ However, this comes at the expense of write speed and so far only yields an areal density improvement of about $25 \%$ and is expected to level at an areal density of $1.5 \mathrm{~T}$ bit per squareinch. The combination with a two-dimensional readout was suggested, ${ }^{26}$ but has not been introduced in a commercial product yet. He-filled HDD chassis with improved thermal properties do not address any of the above issues, but simply allow for more platters in one hard drive, thus increasing the storage capacity ${ }^{27}$ while sacrificing reliability (the $\mathrm{He}$ is inevitably leaking from the HDD chassis and thus the device performance decreases over the years).

In the light of these fundamental limitations, it is worth looking at alternatives. Perhaps the most obvious one addresses the fundamental limitation of the saturation magnetisation of the write pole tip. For more than 20 years, FeCo alloys were the material of choice as depicted in Fig. 1, for they combine an outstandingly large saturation magnetisation $M_{\mathrm{S}}$ with high Curie temperature $T_{\mathrm{C}}$, while being magnetically soft, assuring steady device operation and high writing speed. No other bulk material was ever found which could compete with high-moment FeCo alloys at room temperature. But does this mean there are no alternatives? Probably the most exotic of all new hard drive concepts was patented by Seagate Technology in 2006: ${ }^{29}$ thermoelectrically cooled write pole tips made from ultra-high magnetic moment rare earth (RE) metals rather than Fe-based transition metals (TMs). This is one possible material class with larger magnetic moment than $\mathrm{FeCo}$, which received comparatively little attention but that could potentially improve the HDD performance by increasing the write-field strength and hence breaking the trilemma. Important prerequisites are a sufficiently cooled pole tip, which is kept in an inert atmosphere such as $\mathrm{He}$, to prevent the highly reactive REs from degrading.

This article reviews possible material candidates for high-saturation-magnetisation applications. The focus here is 
primarily for use in magnetic recording write poles, and also for use in other electromagnets such as in superconducting solenoids for creation of giant magnetic fields, operating at cryogenic temperatures. The development of $\mathrm{FeCo}$ is presented as a starting point and a reference, followed by other high-moment TMs, heavy RE metals for use at cryogenic temperature, and combinations of the two in form of alloys and thin-film laminates. The latter are particularly relevant because continuous and ongoing reduction in device dimensions paves the way for potentially promising high-moment material systems such as ultrathin films and nanoclusters, both of which are included in this review. Although write pole dimensions are presently already in the nanometre regime ${ }^{30}$ they are still big enough to have magnetic properties be comparable to bulk systems. Finite-size effects such as epitaxial strain and granularity, however, were shown often to reduce magnetic moments; hence, we will indicate if magnetic properties are different between thin film $(t \sim 100 \mathrm{~nm})$ samples and bulk samples, in cases where data is available. With crystal structure being of pivotal importance to the magnetic properties, the article includes a brief review of thin-film fabrication techniques and their applicability to create high-moment materials. Different ways to specifically cool down solid micro- and nanostructures to cryogenic temperatures are briefly reviewed in Section IG.

\section{A. Magnetic moment and saturation magnetisation}

The saturation magnetisation $\left(M_{\mathrm{S}}\right)$ of a magnetic material is the upper limit of its magnetisation $M$, i.e., its capability to be polarised by an external magnetic field $H$ and thus contributing to the overall flux density: $B=\mu_{0}(H+M)$. For convenience, we chose to give the magnetisation throughout this study as the magnetic polarisation $\mu_{0} M$ whose unit is Tesla (for comparison and conversion to CGS unit, see Ref. 31; see also Ref. 1 in Ref. 32; a rule of thumb for converting magnetic polarisations from CGS to SI is: $\left.800 \mathrm{emu} / \mathrm{cm}^{3} \approx 1 \mathrm{~T}\right)$.

Only FM materials do have a large positive magnetisation and saturate mostly already in moderate magnetic fields. The contribution of their magnetisation to the overall flux density can be drastic: in a $\mathrm{Cu}$ solenoid operating at room temperature the flux density without insertion of a soft $\mathrm{Fe}$ core is only a couple of milli-Tesla, but can be as high as $\approx 2 \mathrm{~T}$ due to the magnetisation of the $\mathrm{Fe} .{ }^{33}$ In contrast to the FM case, paramagnetic (PM) materials only show a moderate increase in magnetisation with applied field, AFM ones remain (nearly) neutral, and diamagnetic (DM) ones polarise negatively. The strongly temperature-dependent positive polarisability of ferrimagnetic (FIM) materials is dependent on the alloy composition but cannot reach FM quality. ${ }^{34}$ Permeability, defined as $\mu=\frac{B}{H}$, is a common measure for describing how a given material amplifies or reduces an externally applied field $H$.

Although a classic-macroscopic description of the magnetic polarisability $\mu_{0} M_{\mathrm{S}}=J_{\mathrm{S}}$ is completely sufficient for understanding the working principle of a $\mathrm{Cu}$ solenoid, the quantum-mechanical-microscopic picture has to be considered for more sophisticated material design. In the case of (soft) FM materials, there is a veritable jump in the magnetisation - from zero to saturation - as a result of the alignment of the spontaneous magnetic moments in the direction of the applied magnetic field. These are the magnetic moments $(\mu)$ of individual atoms whose sum yields the total magnetic moment $(m)$ as depicted in Fig. 3. Magnetisation is the total magnetic moment per unit volume. Although in a ferromagnet exchange coupling between adjacent atoms promotes collinear alignment of their spontaneous magnetic moments, any macroscopically large (soft) FM material breaks down into smaller microscopic domains with opposing magnetisation if no external field is applied, in an effort to minimise the total internal energy. The external field which is required to saturate an FM specimen depends on its orientation with respect to the preferred magnetisation direction of the sample, its easy axis. This direction of easiest polarisability is determined by the magnetic anisotropy of the magnetic material, which in turn is dependent on the material class, crystal structure, and morphology.

In the quantum-mechanical picture, individual magnetic moments are a result of the total angular momentum $J$ of unpaired electrons on the outer shells of the atom. The total angular momentum consists of two components: $J=L+S$, where $L$ is the orbital momentum and $S$ the spin momentum. Depending on the material class, i.e., the atomic structure, the contributions of $L$ and $S$ to $J$ are different. This impacts on the overall magnetic moment, and also on other intrinsic magnetic properties like softness, which generally suffers for larger $L$ contributions due to larger magneto-crystalline anisotropy.

Only certain pure elements in the periodic table fulfil the requirement of unpaired "magnetic electrons" in an incomplete $d$ or $f$ shell. Those elements can be divided into four material classes: the iron group $(3 d)$, the lanthanide group (4f), the platinum group $(5 d)$, and the actinide group $(5 f) .{ }^{34}$ In the applied context, only two of these FM material classes are relevant: $\mathbf{3} \boldsymbol{d}$ TMs and $\mathbf{4} \boldsymbol{f}$ heavy REs, whereas $3 d$

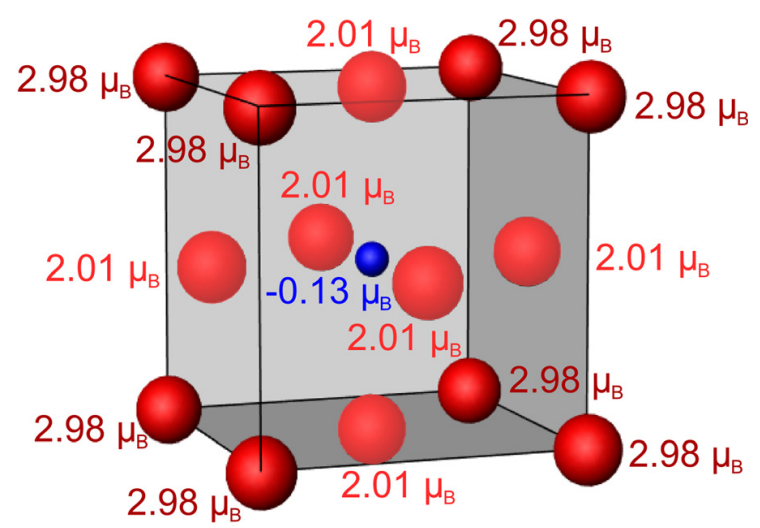

FIG. 3. Depiction of an $\mathrm{Fe}_{4} \mathrm{~N}$ unit cell with numbers indicating the magnetic moments of the individual atoms. While $\mathrm{N}$ is DM and therefore only being weakly negatively polarised, the Fe atoms order FM and thus have a large positive moment. Depending on their position in the unit cell, they are either weak or strong ferromagnets due to charge transfer and thus have a lower or higher moment, respectively. The total sum of all moments within the unit cell is $m_{0}=8.88 \mu_{\mathrm{B}} \mathrm{fu}^{-1}$ which corresponds to a magnetic polarisation of $\mu_{0} M_{\mathrm{S}}=1.89 \mathrm{~T}$. Values for the atomic moments are taken from Ref. 35 . 
and $4 f$ refer to the electron orbit which carries the majority of the magnetic moment. Where for TMs the vast majority of the magnetic moment comes from the spin contribution, making them inherently soft, for REs the orbital contribution cannot be neglected (except for Gd) and causes particularly polycrystalline specimen to exhibit huge coercivities in the ferromagnetic regime. Other materials such as $\mathrm{Pt}, \mathrm{Pd}, \mathrm{Ru}, \mathrm{Rh}$ (of the platinum group), and Sc are considered nearferromagnets since they nearly fulfil the Stoner criterion and readily develop a spontaneous magnetic moment in close proximity to other magnetic atoms in alloys and multilayered thin films or at interfaces in general (see Section IC).

For further reading on the fundamentals of magnetism, we recommend the textbooks by Blundell ${ }^{36}$ and Getzlaff. ${ }^{37}$ A very accessible introduction to magnetism, specifically written for applied scientists and engineers, is the textbook by Cullity and Graham ${ }^{33}$ and Coey's standard volume ${ }^{35}$ serves the more experienced researcher.

\section{B. A brief introduction to first-principle based computational techniques: Density Functional Theory (DFT)}

Modern computational techniques, coupled with the advance in computer hardware, have enabled remarkably accurate modeling and prediction of magnetic moments and magnetisation densities of magnetic materials. Because magnetism is inherently a quantum mechanical phenomenon, predictive models must necessarily be based on quantum mechanics. The theoretical framework used in first-principle based computational modeling of magnetic materials is, almost without exception, DFT. We note that the terminology "first-principle based" means that there are no adjustable parameters in the theory-the only input are fundamental properties of atoms and electrons.

The idea and fundamental principle behind DFT is that the (electron) density can be used as the independent variable from which all other properties can be derived or obtained. This is enormously important from a computational point of view: using density as the independent variable one works with a single scalar variable in three dimensions rather than $N$ quantum mechanical objects, where $N$ is the number of electrons in the problem. DFT is based on the HohenbergKohn theorem, ${ }^{38}$ the essence of which is that the ground state density of a system uniquely determines all properties of the system. This lays the foundation for seeking the ground state density as the strategy for solving quantum mechanical materials problems. The Hohenberg-Kohn theorem also says that the kinetic energy and interaction energy (i.e., the energy from the Coulomb interactions between electrons) of a system of particles is a unique functional $F[\rho]$ of the density $\rho$ that does not depend on whatever external potential to which the system under consideration is subjected. [A functional is a scalar variable that depends not only on the value of the dependent variable (in this case density) at a single point but also on the value of the dependent variable in all space. A simple example of a functional of density is an integral of density over all space.] Of course, most commonly, the interacting particles are the electrons of a material and the external potential is the potential from the atomic nuclei. Finally, the Hohenberg-Kohn theorem provides a variational principle that says that the energy

$$
E_{(v, N)}=F[\rho]+\int v(\mathbf{r}) \rho(\mathbf{r}) d \mathbf{r}
$$

where $v(\mathbf{r})$ is the external potential, is minimised by the ground state energy of $N$ electrons.

The strategy is then to formulate an expression for the energy as a functional of density and a computational scheme to calculate the ground state density and energy. This is what Kohn and Sham did in $1965 .{ }^{39}$ Kohn and Sham, and also von Barth and Hedin, ${ }^{40}$ extended the original Hohenberg-Kohn theorem and the computational strategy to include spin densities, which is the foundation for all the DFT calculations of magnetic systems. They constructed a fictitious single-particle system such that it generates the same ground state spin densities as the interacting system of interest. If the ground state spin densities of the fictitious system can be calculated accurately, and if the energy functional for the interacting system is known accurately, then the ground state energy of the interacting system can be calculated. The key features of the Kohn-Sham (KS) equations, which are the equations that were generated by Kohn and Sham, are that they look like the Schrödinger equation for $N$ non-interacting electrons with a kinetic energy term, a classical Coulomb interaction, an interaction with the external potential, and a final potential term, the so-called exchangecorrelation potential. This last one contains the bridge between the energy of the non-interacting fictitious system and the real interacting system and includes all quantum mechanical effects beyond (single-particle) kinetic energy and classical Hartree-Coulomb interactions, and this is where all important approximations are made, and it is also the source of inaccuracies and errors.

The first implementations of the KS equations were based on the Local (Spin) Density Approximation (LDA or LSDA). The idea was to approximate the exchange correlation energy by a local function $\epsilon_{x c}\left(\rho_{\uparrow}, \rho_{\downarrow}\right)$ of the spin-up and spin-down electron densities $\rho_{\uparrow}, \rho_{\downarrow}$. The value for $\epsilon_{x c}\left(\rho_{\uparrow}, \rho_{\downarrow}\right)$ is then obtained from a homogeneous electron gas with spin densities $\rho_{\uparrow}, \rho_{\downarrow}$, and the total exchange-correlation energy $E_{x c}\left[\left(\rho_{\uparrow}, \rho_{\downarrow}\right)\right]$ is calculated as

$$
E_{x c}\left[\left(\rho_{\uparrow}, \rho_{\downarrow}\right)\right]=\int \rho(\mathbf{r}) \epsilon_{x c}\left[\rho_{\uparrow}(\mathbf{r}), \rho_{\downarrow}(\mathbf{r})\right] d \mathbf{r},
$$

with $\rho(\mathbf{r})=\rho_{\uparrow}(\mathbf{r})+\rho_{\downarrow}(\mathbf{r})$. While the LSDA has been tremendously successful, partly because it is simple and partly because it is based on a real physical system (the uniform electron gas), it also has shortcomings. It is well-known that this approximation is not particularly good for materials where electronic correlation effects become important, and this includes in many cases TMs, especially TM oxides, where the more localised $3 \mathrm{~d}$ electrons are not well described by the uniform electron gas approximation. Furthermore, the LSDA notoriously under-estimates the electronic band gap for semiconductors (SCs) and insulators. Nevertheless, the LSDA is surprisingly accurate in determining the structure 
and magnetic properties (spin densities) of ferromagnetic TMs.

The most common implementations of DFT currently use an extension of the LSDA, the Generalised Gradient Approximation (GGA) which, in addition to the local values of the spin densities, includes gradient terms in the spin densities. ${ }^{41}$ The GGA still underestimates band gaps and can give dramatically incorrect results for correlated materials, but it typically improves structures over the LSDA (the LSDA tends to overbind materials with smaller interatomic separations by $1 \%$ or so than measured in experiments, while GGA tends to very slightly underbind). Also, of importance for magnetic materials, the GGA typically gets the magnetic moment and magnetisation density of TM ferromagnets to within a few percent.

There are now plenty of high-performance computer codes that implement DFT. Most modern codes now use pseudopotentials instead of the bare potential of the atomic nucleus stripped of all its electrons. The reason for this is that pseudopotentials allow for much more efficient calculations. The core electrons, which do not take part in lowenergy processes near the Fermi energy, are excluded so fewer electrons need to be included in the calculations. Also, valence and conduction electron states have to be orthogonal to core electrons. Therefore, if core electrons, which are localised near the nucleus, are included, the valence and conduction electron wavefunctions oscillate very rapidly near the nucleus with the result that much higher spatial (or energy) resolution is needed than if the core electrons are excluded, in which case valence and conduction wavefunctions can be much smoother near the nuclear positions.

For heavier elements, spin-orbit coupling becomes important, and the orbital moments of the electrons can give appreciable contributions (both positive and negative) to the total magnetic moment or magnetisation density, as discussed earlier. Most codes now have options to use fully relativistic pseudopotentials that include spin-orbit coupling. An added complexity in these calculations is that the electronic states are now spinors and the spin density is not necessarily collinear (only up- and down-spin components) but is a full $2 \times 2$ matrix.

\section{Stoner criterion}

The exchange interactions between electrons are responsible for the emergence of ferromagnetism. Even if the exchange interactions are not strong enough to cause a phase transition from a PM state to a FM one, they can significantly enhance the PM susceptibility. There is a mean-field theory due to Stoner that expresses this in a simple way. The theory starts by considering the occupancy $n_{i, \sigma}$ of an electron of spin $\sigma$ at a site $i$, and writing it as a sum of an average occupancy $\left\langle n_{i \sigma}\right\rangle$ and a fluctuation $\delta n_{i \sigma}$. For FM systems, the theory then proceeds by assuming that the average $\left\langle n_{i \sigma}\right\rangle$ is independent of the site $i$. This is a crucial assumption that makes the theory tractable. Next, one substitutes $n_{i \sigma}=$ $\left\langle n_{\sigma}\right\rangle+\delta n_{i, \sigma}$ in expressions for the electron-electron interactions, ignoring terms of second order and of higher fluctuations. By using the relations between average electron density $\rho=\left\langle n_{\downarrow}\right\rangle+\left\langle n_{\uparrow}\right\rangle$ and average spin polarisation $m=$ $\left\langle n_{\uparrow}\right\rangle-\left\langle n_{\downarrow}\right\rangle$ one can then derive self-consistent equations for the chemical potential $\mu$ and the spin polarisation $m$. This eventually leads to an expression for the FM susceptibility $\chi=M / H$

$$
\chi=\frac{\chi_{0}}{1-I}=\frac{\chi_{0}}{1-U / U_{c}} .
$$

Here, $\chi_{0}$ is the Pauli susceptibility $\chi_{0}=\mu_{B}^{2} \mathcal{N}\left(\epsilon_{F}\right)$ with $\mu_{B}$ being the Bohr magneton and $\mathcal{N}\left(\epsilon_{F}\right)$ the density of states at the Fermi energy; $U$ is a parameter for the electron-electron interaction strength, and $U_{c}=2 / \mathcal{N}\left(\epsilon_{F}\right)$. The quantity $1 /(1-I)$ in Eq. (3) is called the Stoner enhancement factor. If $I$ in Eq. (3) approaches or exceeds unity, the susceptibility diverges, which signals the onset of spontaneous magnetisation, i.e., ferromagnetism. However, the susceptibility can be enhanced appreciably over its Pauli value if $I>0$ but still remain smaller than unity, thus not fulfilling the Stoner criterion. This is the case for elements such as $\mathrm{Pd}, \mathrm{Pt}, \mathrm{Ru}, \mathrm{Rh}$, and Sc that are not FM but have an enhanced susceptibility because of the Stoner enhancement. ${ }^{35}$ This is also the reason that these elements develop appreciable moments in proximity to FM elements, such as Co or Fe.

\section{High moment materials: The benchmark case of the HDD write pole tip}

Magnetic materials with large (saturation) magnetisation are mainly used to amplify an existing magnetic field, with the prime example being the common electromagnet. A solenoid wound from $\mathrm{Cu}$ wires generates a weak primary field [Fig. 4(a)], but by insertion of a soft Fe core the flux density is increased up to three orders of magnitude ${ }^{33}$ [Fig. 4(b)]. The desired output field $H_{\mathrm{ag}}$ for writing on the recording medium is generated in a small air gap $d_{\mathrm{ag}}$ of the Fe yoke. (a)

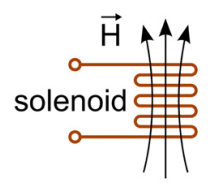

(b)
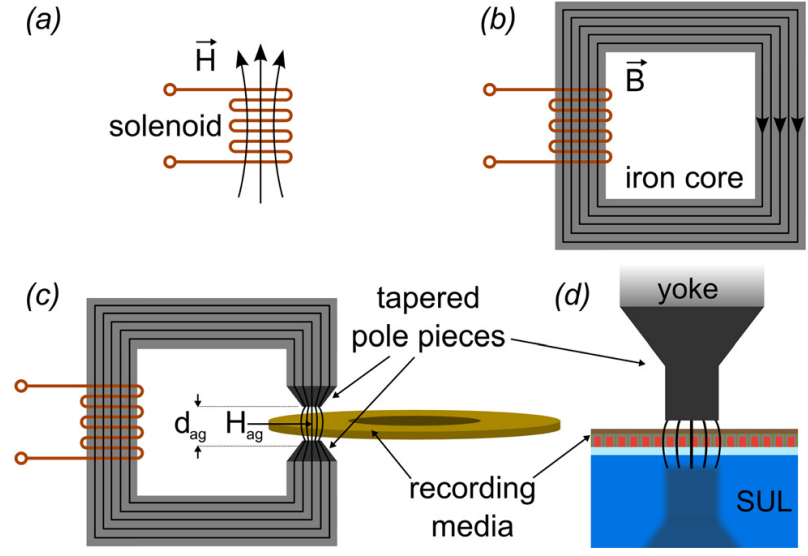

FIG. 4. Solenoids with increasing magnetic field strength. (a) Simple solenoid in vacuum. (b) Solenoid with Fe core whose magnetisation contributes to the overall flux. (c) Magnetic recording setup: the recording media is placed within a small air gap in the Fe core and tapered pole pieces further increase the flux density geometrically. (d) A soft underlayer (SUL) below the recording media, magnetically mirroring the tapered pole piece (i.e., the write pole tip in a HDD) allows for better flux confinement in the recording layer and thus minimised gap distances of $d_{\mathrm{ag}}<10 \mathrm{~nm}$. 
To boost the output even further the air gap is confined by tapered pole pieces, which allow for an increase of the flux density by funelling the flux into a smaller cross-sectional area [Fig. 4(c)]. The maximum output field $H_{\mathrm{ag}}^{\max }$ is proportional to the system's maximum flux density ${ }^{42} \stackrel{\mathrm{ag}}{\mathrm{max}}$ and thus largely determined by the magnetic properties of the pole tips, particularly their magnetisation $M$ in the field of the solenoid $H$. The saturation magnetisation can therefore be considered the bottleneck of the system: once the pole tips saturate, i.e., are fully polarised, $H_{\mathrm{ag}}$ reaches its maximum value. In other words, to further increase $H_{\text {ag }}$ one has to find a material with a larger $M_{\mathrm{S}}$ (provided the external field is enough to fully saturate).

These fundamental design considerations are also the working principle behind the pole tip of a modern-day HDD, with the dimensions of the pole tip being in the nano-regime (see, for instance Refs. 24, 30, and 43-46). Due to today's unprecedented demand for cheap storage space, in conjunction with outstanding research efforts, the HDD write pole became somewhat of a role model for pole piece development, both in terms of material design and device integration by miniaturisation. This move to nano-dimensional structures was not solely based on considerations to increase the storage capacity by shrinking the bit sizes and concomitantly the write pole crosssectional area, but unlike thin-film write heads, (ferrite) dual-pole longitudinal write poles suffer from saturation effects in the gap corners. To boost the flux density even further, current HDD write heads utilise perpendicular recording, in which the recorded bits in the recording layer on the disk are magnetised perpendicularly to the plane of the disk, and a single-pole design. This technological advance was enabled by the implementation of a soft magnetic underlayer (SUL) beneath the recording layer, which acts as the return path for the magnetic flux and hence allows for a very compact device design [Figs. 2(b) and 4(d)]. With the air gap size thus being kept below $10 \mathrm{~nm}$, larger effective write fields of about $\mu_{0} H_{\mathrm{ag}} \approx 1.7 \mathrm{~T}$ can be achieved [assuming pole tips from $\mathrm{FeCo}$ and a gap distance of about $10 \mathrm{~nm}$ (Ref. 35)]. Yet again, the need for write pole materials with a larger saturation than the currently used FeCo has not ceased and the "trilemma" dictates the problem: smaller bits ultimately necessitate $\operatorname{larger} M_{\mathrm{S}}$. This need would persist ${ }^{47}$ even without using a recording medium with increased $H_{\mathrm{C}}$ : shrinking the bits demands a larger $M_{\mathrm{S}}$ for a well-confined $H_{\mathrm{ag}}$ with large field gradient to write sharp bits. ${ }^{20}$

The main requirements a high-moment pole piece material needs to satisfy can be divided into primary magnetic properties:

- $T_{\mathrm{C}} \gg T_{\mathrm{op}}$ : A sufficiently high Curie point $T_{\mathrm{C}}$ assures high and stable $M_{\mathrm{S}}$ at operating temperature $T_{\mathrm{op}}$ (although actively cooling the write pole might help to bypass this requirement as for superconducting solenoids operating at liquid He temperatures).

- High magnetisation $M$ : A strong amplification of the externally applied field allows writing smaller bits, even if they have a large coercivity.
- Large saturation magnetisation $M_{\mathrm{S}}$ : The uppermost limit of that amplification, setting the maximum value of the flux, i.e., the write field.

\section{and secondary magnetic properties:}

- Softness, with the coercive field strength $H_{\mathrm{C}}$ being the quickly accessible quantity: Easing the requirements on the primary field (as induced by the solenoid current).

- High initial permeability: Allowing for fast magnetisation reversal and thus high operating frequency.

- Favourable magnetic anisotropy: Aiding the confinement of the magnetic field and assuring lowest possible permeability.

- Small remnant fields: avoiding undesired writing events.

- Low magneto-striction: supporting a stable device operation and aiding softness. ${ }^{48}$

- Compatibility to adjacent materials: There is no benefit in having a phenomenal write pole material in the lab which cannot be integrated in an actual write head or which looses its superior properties within a couple of hours due to interdiffusion with its surrounding.

- Corrosion resistance for long-term stability.

The secondary properties are not of pivotal importance but nevertheless need to be considered in the final device design. For instance, if the source of the primary magnetic field is only an air-cooled $\mathrm{Cu}$ solenoid, a particular highmoment candidate with a low initial permeability and large coercivity is hardly an option, since its magnetisation at the available field strength might only be a fraction of its saturation. This is especially a problem for next-generation HDDs operating at $\mathrm{GHz}$ frequencies: with each writing event taking only a few nanoseconds and less, a critical regime is reached in which any polycrystalline (or nanostructured) FM material experiences a drastic non-linear increase in coercivity. ${ }^{49}$ Thus, even moderately soft pole piece candidates might not actually reach their saturation and deliver only insufficient write field strength operating at or beyond their roll-off frequency.

A possible way to bypass this issue might be a tiered pole piece with a multi-stage flux concentrator, with the inital stage (after the yoke) being made of a soft high moment material, such as $\mathrm{FeCo}$, followed by the harder candidate (at the air gap side), such as $\alpha^{\prime \prime} \mathrm{Fe}_{16} \mathrm{~N}_{2} .{ }^{50}$ If the harder material is an RE such as Dy, an exchange break needs to be inserted to avoid antiparallel coupling of the soft initial stage TM and the subsequent RE (see discussion in Section IIG). This exchange break could be as simple as an air gap or, for instance, could be made from a suitable oxide or SC barrier with decent crystal structure match. Alternatively, the write pole tip itself could be heated prior to the writing event, in order to make it softer to reduce the critical field required for saturation. This process, however, is limited by the cooling rate of the system and might require a sophisticated heat sink design. Only when the pole tip returns to sufficiently low temperatures again, the magnetic moment will be high enough. This approach, of course, is hardly suitable for thermally unstable materials such as $\alpha^{\prime \prime} \mathrm{Fe}_{16} \mathrm{~N}_{2}$ and, if an inert atmosphere cannot be guaranteed, also highly reactive RE metals. 


\section{E. Fabrication techniques suitable for the creation of magnetic (ultra-) thin films and nanostructures}

Throughout the review, much attention is paid to different fabrication techniques, for they largely dictate the resulting layer structure and, therefore, also its magnetic properties. Thin-film deposition techniques can in principle be divided into four main classes: electroplating (also electrochemical deposition or electrodeposition), ${ }^{51}$ electroless (chemical) deposition, ${ }^{52}$ chemical vapour deposition (CVD), ${ }^{53}$ and physical vapour deposition (PVD). ${ }^{54}$

\section{Electroplating and electroless deposition}

Historically, electroplating was the first technique to become available and was broadly studied. ${ }^{55}$ Layer structure can be controlled by deposition parameters such as temperature, applied current or voltage (including the possibility to use pulses ${ }^{56}$ ), electrode geometry, and electrolyte composition-but layers will inevitably be polycrystalline resulting in a comparatively high surface roughness. ${ }^{57}$ Inclusions in the final film due to two stabilising agents in the solution also often have undesired side-effects, but are a necessary requirement. It was shown that the magnetic structure of electroplated thin films can be influenced by applying a magnetic field during the deposition process. ${ }^{58}$ Although electrodeposition is comparatively cheap and easy to scale, the limited control of layer properties on the nanoscale makes it primarily a suitable choice for thicker magnetic films in the micrometre regime. Electroplating poses a serious challenge when attempting to deposit pure RE metals due to their very negative reduction potential. ${ }^{59-61}$ An excellent review on structural and magnetic properties of electroplated TMs (the FeCoNi system plus various additives) for use in write pole tips is given by Cooper et al.; ${ }^{3}$ see also Liu and coworker. ${ }^{62}$

Electroless deposition follows the same principle of chemical reduction of the to-be deposited material from a precursor like electroplating, but the chemical reactions occur without the need for additional electrons being provided by an electrical current. ${ }^{63}$ The obvious disadvantage over electroplating is limited reaction speed and perhaps a certain lack of control. The advantage, however, is the much smoother profile of the final film because the method does not suffer from "dog-boning". 64 The most suitable electroless plating method for ultra-thin layers is dip-coating. Although its name might suggest otherwise, it is a high-end method which, when conducted carefully, can coat a workpiece with as little as a monolayer (ML) of material and is also scalable. A large disadvantage is the occurrence of reaction by-products, which end up as impurities in the final layer and thus, as with electrodeposition, generally prevents the creation of pure metallic thin films of epitaxial quality ${ }^{65}$ and limits dip-coating mostly to the creation of organic monolayers. Besides self-triggered reactions, there is also those being triggered by photon-energy in photodeposition but there is no benefit over electrodeposition in terms of controllability, scalability, or reaction speed nor does the method allow for the creation of epitaxial thin metal films - although the method has already been used in the creation of magnetic recording material in the past. ${ }^{66}$

\section{CVD}

CVD processes so far only exist at the periphery of magnetic material fabrication, such as for creating the protective overcoat from carbon for HDDs by plasma-enhanced CVD. ${ }^{67}$ The term CVD hosts a variety of different processes, all of which are based on a volatile precursor reacting on the surface of the substrate. The technology has not found its way into large-scale fabrication of magnetic thin films and, since it has not shown significant advantages over wellestablished PVD until now, it appears unlikely to ever rise to importance. The absence of shadowing effects, i.e., a more homogeneous profile of the deposited material, might be considered a major advantage; however, inclusions of the reaction by-products cause undesired defects which are a major disadvantage, particularly when working with RE metals. Atomic layer deposition (ALD), as one of the most prominent examples of CVD due to its outstanding level of control over the chemical reaction at the substrate, has already found its way into recording head fabrication, but only for the creation of conformal layers of insulators $\mathrm{Al}_{2} \mathrm{O}_{3}$. A lot of progress has been made in the synthesis of metallic layers over the last 15 years, with ALD of TMs such as Ni and $\mathrm{Co}$ (and $\mathrm{Cu})^{68}$ and noble metals (NMs) such as Ru and $\mathrm{Pt}^{69}$ being highly relevant to the field. The major disadvantage of ALD is the slow reaction speed, making it unsuitable for the creation of layers which exceed a few nanometres, as would be required for present day write pole tips. However, when aiming for ultrathin films, ALD might be of interest again, given the right precursors for the reaction can be found and impurities in the final layer can be reduced to an absolute minimum.

\section{PVD}

Plasma-supported sputtering deposition (often just called sputtering) is currently the most broadly used thinfilm PVD technique, for it allows reliable and quick largescale fabrication of uniform layers of high quality. ${ }^{70,71}$ The deposition rate can be controlled by changing the applied power and the process gas pressure (mostly Ar). Strong permanent magnets often aid the plasma formation by increasing the effective pathway of the electrons within the plasma, referred to as magnetron sputtering. Using different operating modes, such as direct-current (DC) and radio-frequency (RF), allows fine-tuning of the layer structure. ${ }^{72}$ The ability to sputter from several targets at once (co-sputtering) - as well as to inject different gases such as $\mathrm{O}_{2}$ or $\mathrm{N}_{2}$ for reactive sputtering - enables the creation of a diverse range of alloys, compounds, and laminates. Cooling the substrate during deposition effectively reduces the crystallite size, whereas heating the substrate was shown to be an effective means to support the formation of the equilibrium phase and to increase the crystallite size up to several microns. Similar effects were achieved by supporting the layer formation by scanning with a directed Ar beam across the substrate during deposition, called ion-assisted sputter deposition. ${ }^{73}$ An 
additional degree of freedom is given by the angle of incidence of the ablated target material with respect to the substrate surface, which is used in oblique sputtering. ${ }^{74}$ And in-situ field annealing has proven a most powerful tool in manipulating the magnetic structure immediately during fabrication, often increasing softness and aiding in pre-defining the magnetic easy axis. ${ }^{75}$

Alternatives which yield similar layer quality, but which are less prominent, are for instance pulsed-laser deposition (PLD), electron-beam deposition, ion-beam deposition, and thermal evaporation. ${ }^{76}$ PLD is broadly used in the creation of high-uniformity oxide layers, where it can exceed the quality of reactively plasma-sputtered films. Electron-beam deposition is a highly directed deposition method and thus allows for sharp edges, making it the industry standard for delicate lithographic tasks, and it also yields superior layer quality when compared to sputter deposition due to the lower deposition speed. However, in addition to the limited deposition speed scalability is also hampered. In general, sputtering is deemed the best method for the creation of high-quality pure metal layers with an eye on profitability (i.e., scalability) since the introduction of magnetrons, which greatly increased the deposition rate while reducing the inclusions and layer defects to a minimum. ${ }^{77}$

Molecular beam epitaxy (MBE) might not be desirable from a financial point of view, but considering the unrivalled layer quality, industry might eventually be forced to reluctantly follow a new paradigm. ${ }^{78}$ Only MBE-grown films of $\mathrm{FeN}$ were shown to stabilise the high-moment $\alpha^{\prime \prime} \mathrm{Fe}_{16} \mathrm{~N}_{2}$ phase $^{79}$ and for ultra-thin films or laminates, which require defect-free and atomically sharp interfaces, there is also no other viable choice at present. Being used to deposit only ultrathin layers and seeds, otherwise time-consuming MBE could be integrated in existing high-yield PVD systems to significantly improve layer quality.

\section{Seed layers and strain engineering}

The texture of any thin film is nowadays determined and stabilised not only by the substrate but also by using seed layers (and capping layers) with appropriate crystal and electronic structure. The three most common crystal structures among magnetic materials are body-centered cubic (BCC), face-centered cubic (FCC), and hexagonal close-packed (HCP), which (when deposited on an amorphous substrate) typically have a texture which allows for the largest atomic density at the interface plane, i.e., (l 110$)$, (ll $\left.1 \begin{array}{ll}1 & 1\end{array}\right)$, and (l $\left.\begin{array}{lll}0 & 0 & 2\end{array}\right)$, respectively. Not uncommonly, BCC materials are strained, forming a body-centered tetragonal (BCT) phase at the substrate interface with a more symmetric atomic arrangement in the $\left\{\begin{array}{lll}1 & 1 & 0\end{array}\right\}$ plane, and HCP materials can develop a $\left(\begin{array}{lll}1 & 1 & 1\end{array}\right)$ textured FCC phase, since its atomic order in the $\left\{\begin{array}{lll}1 & 1 & 1\end{array}\right\}$ plane is virtually identical to the HCP $\left\{\begin{array}{lll}0 & 0 & 2\end{array}\right\}$ one-only the stacking order along the $c$-axis differs. ${ }^{80}$ But by choosing the right set of seed materials forming a stack beneath the magnetic layer (being deposited under the right conditions) one can synthesise nearly any material in a large variety of textures and versatile crystallinity. Seed layers are also a powerful tool in adjusting the interatomic spacing via epitaxial strain, inducing a deliberate symmetry break in the crystal lattice thus changing the magneto-crystalline anisotropy or even the entire magnetic ordering. Prominent examples for strain engineering are the growth of highly anisotropic $\mathrm{L} 1_{0} \mathrm{BCT}$ phases of $\mathrm{CoPt}$ and FePt alloys, proposed for future HAMR recording media. ${ }^{47}$ However, finding the right seed stack composition can be vastly time consuming and sensitively depends on deposition method and parameters. In the special case of magnetic materials, the choice of the right seed material is also influenced by its compatibility with the magnetic layer-their interaction at the seed interface heavily influences the overall magnetic properties. One example is $\mathrm{Pd}$ seed layers: Pd increases the magnetic moment in adjacent TMs, but has little to no influence on adjacent REs. Wrong seed materials can even harm the magnetisation of a thin film, by causing reduced or even completely suppressed spontaneous magnetic moments in adjacent monolayers of the magnetic material due to hybridisation-effectively turning them into magnetically dead layers.

\section{Concluding remarks}

Regardless of the fabrication method, the stabilisation of the right crystal phase with the desired ordering has always been challenging. FeCo alloys are also the pole-piece industry standard for the ability to control their crystallisation well, but also the robustness of the magnetic moment, which seems to change only marginally for different fabrication techniques and resulting structures, nowadays optimised for increased softness and low magnetostriction. Promising new material candidates, such as ultra-thin TM films or TM-Pd laminates (see Section IIB), RE/IE/TM, and RE/Cr/TM stacks (see Section IIG3) or $\alpha^{\prime \prime} \mathrm{Fe}_{16} \mathrm{~N}_{2}$ (see Section IIE), however, tend to order in a way not beneficial to obtaining a useful high moment, i.e., the crystallite orientation of the thin film does support a magneto-crystalline anisotropy with an out-of-plane easy axis. This makes them effectively harder and contributes to undesired pole-erasure and crosstalk of the write pole if its design is not changed, since present day fabrication of the pole tip relies on the FeCo thin film's in-plane direction being the easy axis. An easy implementation in current write heads of next-generation high-moment materials, even if their high moment phases are reliably stabilised, is therefore highly unlikely unless their thin-film growth produces the right crystallite orientation or write head fabrication and design are adapted. Such a high level of control over crystal formation is most likely realised by $M B E$, however at the expense of scalability, and certainly requires additional comprehensive studies on alternative substrates and seed layers. An overview of the most important thin film fabrication techniques introduced in this section is given in Table I, with an assessment of their suitability for creating magnetic thin films-with magnetron sputtering currently presenting the best trade-off between layer quality and cost efficiency. 
TABLE I. Assessment of different fabrication techniques for their potential in creating magnetic thin and ultra-thin films.

\begin{tabular}{|c|c|c|c|c|c|}
\hline Fabrication technique & Cost efficiency & Scale-ability & Yield & Control-ability & Layer quality \\
\hline Electroplating & +++ & ++ & +++ & + & - \\
\hline Electroless plating & +++ & ++ & - & - & - \\
\hline Magnetron sputtering & ++ & +++ & +++ & +++ & ++ \\
\hline Pulsed laser deposition & ++ & +++ & +++ & ++ & ++ \\
\hline Chemical vapour deposition & + & + & + & ++ & ++ \\
\hline Atomic layer deposition & - & + & - & +++ & ++ \\
\hline Molecular beam epitaxy & -- & -- & - & +++ & +++ \\
\hline
\end{tabular}

\section{F. Magnetometry: Measuring the magnetic moment and magnetisation}

Among a variety of different technologies to measure the total magnetic moment of a sample two devices have prevailed and are most commonly used in sample characterisation: the Vibrating-Sample-Magnetometer (VSM) and the SuperConducting-Quantum-Interference-Device (SQUID). When the element-specific magnetic moment of an individual atom needs to be identified, X-ray Magnetic Circular Dichroism (XMCD) is the superior, but demanding (and expensive!) method of choice. Ferromagnetic resonance (FMR) cannot compete with either of the other magnetometry methods when investigating static magnetic moments, but is extremely useful for high-frequency measurements.

\section{Magnetometry techniques}

The VSM is the workhorse of magnetometry: it is cheap, quick, reliable, and reasonably accurate $\left(\Delta m<10^{-6} \mathrm{emu}\right.$ $=10^{-9} \mathrm{Am}^{2}$ ). The sample is placed in the desired magnetic field, which is created either by traditional $\mathrm{Cu}$-based solenoids or by much stronger superconducting magnets, and the holder is set in motion. The stray field of the sample is then recorded by nearby pickup coils. ${ }^{81}$

The SQUID is mostly used for more delicate tasks which require a higher accuracy $\left(\Delta m<10^{-8} \mathrm{emu}=10^{-11}\right.$ $\mathrm{Am}^{2}$ ) at the expense of measuring time. At the heart of this technology is a flux transformer operating a superconducting ring with a Josephson junction. The sample's strayfield is recorded by pickup coils which thus couple the flux to the ring, which in turn yields a voltage output. ${ }^{82,83}$

Any measurement of the total magnetic moment of a bulk FM sample needs to be corrected for the demagnetising field due to the shape of the body. Conveniently, in the case of in-plane measurements of thin films the demag field is zero and needs no consideration.

The XMCD requires a source of circularly polarised tunable X-rays - a synchrotron-which makes it a less accessible, high-end analysis but it allows for the identification of the magnetic moment of atoms in individual monolayers with remarkable accuracy $\left(\Delta \mu \approx 0.005 \mu_{\mathrm{B}}\right)$. It might be considered an indirect measurement technique, because not the strayfield is measured, but the electronic structure is identified by the help of X-ray absorption spectra. The difference in the absorption of left and right circularly polarised $\mathrm{X}$-ray radiation gives information on the spin and orbital contribution to the total moment. ${ }^{84}$
Although much less accurate and thus not as widespread, FMR is a common technique to determine the saturation magnetisation of a thin film. It is limited to soft thin films only, due to the penetration depth and field strength of the electromagnetic RF excitation. On the plus side, it does allow for a quick analysis of the (uniaxial and cubic) anisotropy and has been proven to be a powerful tool when looking at more delicate magnetic properties like those of spin waves, exchange bias and damping. ${ }^{85,86}$

\section{On the limits of magnetometry}

Although measuring the total magnetic moment is now considered a standard procedure and can be done with remarkable precision with commercial equipment, accurately determining the magnetisation of a thin film can be challenging. For determining the volume of thin films, one needs to know the film thickness and surface roughness, which is everything but trivial for ultra-thin films (of $t<5 \mathrm{~nm}$ ): neither transmission electron microscopy (TEM) imaging on thin-film cross-sections nor X-ray reflectometry (XRR), which are most commonly used, can guarantee accurate thickness measurements below $2 \mathrm{~nm}$, which becomes a problem in laminated thin films, too. Interdiffusion at interfaces additionally complicates the identification of boundaries and thus the accurate determination of layer volumes. Therefore, instead of magnetisation often values of the total magnetic moment per unit mass are given (see Ref. 1 in Ref. 32 for comparison). This is undoubtedly an accurate measure, but particularly in proximity to interfaces the crystal structure is mostly not isotropic and different monolayers often have different lattice parameters or a different structure altogether, which prevents an accurate derivation of the magnetisation. An additional obstacle to precision is the existence of magnetically dead layers at certain interfaces - one or more monolayers of magnetic material which lost their spontaneous magnetic moment due to hybridisation with a neighbour of unbeneficial electronic structure.

Recalling the model case of an HDD write pole, one is reminded that not only the magnetic moment is important but also how much of it is actually available in a given external field. In the highly dynamic case of $\mathrm{GHz}$ operation of next-generation drives, the attention should turn to the dynamic magnetisation rather than simply the quasi-static one. A great deal of work has been done to understand the dynamical behavior of a magnetic specimen by looking at its permeability at high-frequency magnetic fields with various FMR techniques. ${ }^{87}$ In general, one finds that ferromagnets 
become harder and have lower (initial) permeability due to damping effects slowing down the reversal of the magnetisation as described by the Landau-Lifschitz-Gilbert equation. ${ }^{88}$ But despite a satisfying theoretical description, the complexity of magnetisation dynamics makes predictions quite difficult and demands case to case investigations of each material under consideration. In addition, most of the experimental methods are far from a commercial-grade development and add large errorbars to each measured value.

All the above considerations need to be taken into account when investigating future pole piece materials: they need to stay sufficiently soft and thus keep their large magnetisation also in $\mathrm{GHz}$ operation, i.e., write cycles of only a few nanoseconds and less, and should be thoroughly analysed for their dynamical behaviour best in a spin stand which resembles the application case closest. ${ }^{89}$

\section{G. Cooling solid nanostructures}

The incentive to cool the pole pieces in electromagnets is obvious: the magnetic moment of any FM material becomes larger with reduced temperature because of reduced thermally induced spin disorder (with the exception of FeRh, see Section II B 3). This might not be relevant for bulk FeCo, the high Curie temperature of which results in marginal changes of the magnetisation in the range of $0<T \leq 300 \mathrm{~K}$, but for systems of reduced dimensionality, like ultra-thin films and nanoclusters, thermal effects are particularly pronounced and effectively lower the Curie temperature-and therefore also the possible operating temperature range — often to below room temperature; for RE metals the Curie temperature is below $300 \mathrm{~K}$ in the first place, limiting practical operations of them to cryogenic temperatures. In considering future device integration of nanodimensional pole pieces, it is worth looking at suitable cooling methods other than mechanical refrigeration and cryogenic liquids and evaluating their cooling potential.

As a guideline, we use the heat generation in a presentday write pole during the writing process that brings the pole piece to an operating temperature of up to $T_{\text {op }} \approx 80^{\circ} \mathrm{C}$ within a few microseconds. The reasons for this are the $\mathrm{Cu}$ coils heating up when current runs through them, or effects such as the increased pressure due to the air bearing of the recording head assembly ${ }^{90,91}$ with concomitant increased friction. In the HAMR heads, the temperature will likely be higher due to additional heat from the plasmonic resonator and the need for bringing the head in a thermal equilibrium prior to the writing event. ${ }^{92}$

\section{Magneto-caloric cooling}

The magneto-caloric effect can be understood in terms of an adiabatic change in temperature in magnetic materials due to an applied field, and it is largest near the Curie temperature, because close to the Curie temperature the ordering force due to exchange interaction and the disordering force due to thermal vibrations are approximately balanced. ${ }^{93}$ The giant magneto-caloric effect occurs in materials where magnetic and structural degrees of freedom are particularly strongly coupled, which leads to magnetic phase transitions accompanied by changes in crystal symmetry. ${ }^{94}$ With the discovery of materials exhibiting a giant magneto-caloric effect at room temperature, magneto-caloric cooling was suggested for industrial applications. However, despite intense research ${ }^{95}$ and recent progress in extending the temperature working range of known magneto-caloric materials such as $\mathrm{Gd}^{96}$ or the discovery of new cheaper and better tunable compounds, ${ }^{97}$ there is yet no real application. ${ }^{98}$ However, the giant magneto-caloric effect has so far only been suggested for bulk materials, ${ }^{99}$ and it might be more suitable for nanostructures. A difficult task is the removal of waste heat, e.g., by employing heat conducting fluids, ${ }^{100}$ which is not an option in HDDs.

Operated at room temperature, temperature differences of about $\Delta T \approx 20 \mathrm{~K}$ were achieved for $\mathrm{Gd}^{93}$ but only at field strengths as high as $\mu_{0} H=10 \mathrm{~T}$. For more realistic fields of only $\mu_{0} H=2 \mathrm{~T}$, which can potentially be generated by strong permanent magnets, the temperature difference drops to only $\Delta T \approx 7 \mathrm{~K} .{ }^{101} \mathrm{~A}$ tunable working range around room temperature was shown for $\mathrm{La}_{0.7-\mathrm{X}} \mathrm{Pr}_{\mathrm{X}} \mathrm{Sr}_{0.3} \mathrm{MnO}_{3}$ manganites. ${ }^{102}$ The highest cooling powers were reported for $\mathrm{Gd}$ spheres: $P_{1}=100 \mathrm{~W} \quad\left(\mu_{0} H_{1}=1.5 \mathrm{~T}\right)$ up to $P_{2}=600 \mathrm{~W}$ $\left(\mu_{0} H_{2}=5.0 \mathrm{~T}\right)$ for temperature changes of $\Delta T_{1}=10 \mathrm{~K}$ and $\Delta T_{2}=20 \mathrm{~K}$, respectively. ${ }^{95}$ Low temperature differences, the requirement of large applied magnetic fields, and device integration issues, such as the removal of the waste heat, pose a serious challenge and make it nearly impossible to predict ad-hoc resulting cooling powers in real devices. The magneto-caloric effect is comparatively slow, i.e., even faster second-order magneto-caloric materials (such as $\mathrm{Gd}$ ) only work on the nanosecond scale, whereas the write process happens in the sub-nanosecond regime. ${ }^{95}$ Overall, at the moment this method of cooling appears to be rather unsuitable for application in an HDD.

\section{Giant Peltier effect}

It was found that the Peltier coefficient is larger in nanosized junctions with a current perpendicular-to-plane structure than similar macroscopic structures. ${ }^{103}$ For optimised sub-micron $\mathrm{Cu}-\mathrm{Ni} / \mathrm{Au}$ junctions Sugihara et al. ${ }^{104}$ reported enhancements of the Peltier cooling effect of 40 times over what one would expect from similar materials in bulk structures. They reported a temperature drop of $\Delta T=160 \mathrm{~K}$ and explained it in terms of a nanometre-scale phase separation in the $\mathrm{Cu}$-Ni layer. A surface cooling power of $P \approx 100 \mathrm{~W} / \mathrm{cm}^{2}$ was calculated, which however would only correspond to an effective cooling power of $P_{\text {eff }} \approx 2 \mathrm{nW}$ for a write pole setting.

More commonly, the giant Peltier effect is explained by being part of the wider field of spin caloritronics, ${ }^{105}$ a quickly growing research branch which treats the interaction of spins with heat current in spintronic devices: conventional electric-heat current interactions can be enhanced by directed electron spin manipulation. In terms of applicability, the giant Peltier effect is by far the most promising candidate of all caloritronic effects for cooling nanostructures, but unfortunately lacks additional experimental confirmation. In their 2012 review article, Bauer et al. ${ }^{106}$ summarised: "If 
spin caloritronics is to become more than a scientific curiosity, the thermoelectric figures of merit should be increased. (...) More materials research and device engineering, experimental and theoretical, however, is clearly needed."

Peltier devices in general are the solution of choice for thermo-electric cooling, reaching cryogenic temperatures and high cooling powers, but multistage Peltier elements are simply too big and inefficient to be considered in cooling a write pole tip in a commercial HDD. ${ }^{107}$

\section{Field-emission or thermionic cooling}

Field-emission or thermionic cooling work by removing the "hottest" (most energetic) electrons from the surface of a conductor at an insulator interface (preferably vacuum) by applying an electric field, which is strong enough to increase the tunnelling probability of those hot electrons and hence draws them off the conductor, through the (nanometre-sized) insulator, to a counter electrode. ${ }^{108}$ In 2007, Ng et al. ${ }^{109}$ suggested the use of field-emission energy transfer in an HDD write head design which transfers heat from the write pole to the media. For a real system, operating with air as the insulating material, a major problem is the critical air breakdown-the heat transfer scales with the applied voltage, but for large enough voltages a complete discharge occurs. This limits the applicability of field-emission cooling in real systems. Zhang et al. ${ }^{110}$ evaluated thermo-ionic heating of a $\mathrm{Co} / \mathrm{Pt}$ multilayer system with a scanning tunnelling microscope (STM) tip and found a minimal spot size of $\approx 45 \mathrm{~nm}$ width for voltage pulses with an amplitude of $U_{\max }=5 \mathrm{~V}$. So far, there is no published report on combining laser-based HAMR and field-emission heating but the potential benefit is obvious: applying a moderate voltage between the transducer and the media would allow additional heat transfer of the already very hot electrons to the media, thus supporting the laser heating by this additional energy transfer.

The possibility to employ field emission for cooling the write head was evaluated in a study by Fallon, ${ }^{111}$ who simulated cooling effects for nanometre-sized, well-insulated write poles. Maximum surface cooling powers of $P \approx 100 \mathrm{~W} / \mathrm{cm}^{2}$ were cited, which correspond to a very small effective cooling power of $P_{\text {eff }} \approx 2 \mathrm{nW}$ for the write pole. Therefore, it was concluded that "this cooling power would have practically zero effect on the temperature of the write pole even assuming the best thermal insulators could be used in the recording head."

\section{Optical refrigeration: Laser cooling of solids}

In laser cooling of solids, which is also called antiStokes fluorescent cooling, heat is carried away from the solid by anti-Stokes photons. The solid is illuminated with monochromatic light from a laser source in the red tail of the absorption spectrum of the coolant. The light is absorbed and blue-shifted by phonon absorption, and subsequently emitted. The emitted photons therefore carry away energy from the solid because of the phonon absorption, which cools the solid. Most commonly, two material classes are in use: REdoped solids, mainly Yb-doped glasses or crystals, and SCs, particularly GaAs. The main advantage of this technology is the absence of moving parts and the possibility to cool remote areas (provided that the laser light can access them) without interfering with the electronics of the device. ${ }^{112-114}$ A major challenge is the suppression of reabsorption of already emitted high-energy photons, commonly achieved by strong absorbers placed in immediate proximity to the coolant, which however will become a delicate matter for integration in complex micro- and nanoscale devices.

Laser cooling utilising Yb:YFL crystals was reported to reach cryogenic temperatures down to $T=115 \mathrm{~K}$ and is predicted to reach $T_{\min }=80 \mathrm{~K} .{ }^{115}$ However, the cooling rates (concomitant with the cooling power) were moderate ${ }^{116}$-it took minutes to cool an isolated system without heat source. The setups in use are not scalable down to the micro- or nanoregime and high laser powers of several Watts were necessary to cover $\Delta T \gg 1 \mathrm{~K}$. For SCs, no net-cooling has been reported so far.

\section{Summary: Cooling nanostructures}

At present, none of the above introduced cooling methods alone appears to be a viable option for cooling the write pole in a recording head-or any comparable solid nanostructure integrated in a microdevice:

1. Magneto-caloric cooling cannot cover the required large temperature difference and is hard to implement. All known setups are too big for an HDD or similar devices.

2. Spin caloritronic cooling via the giant Peltier effect yields only modest cooling powers.

3. Field-emission or thermionic cooling also suffers from low cooling powers.

4. Laser cooling can in principle reach the cryogenic regime, but only at high laser powers and after considerable time, i.e., it does have insufficient cooling power (and efficiency).

Research is ongoing, and larger temperature differences of up to $\Delta T \approx 100 \mathrm{~K}$ might be achieved for Gd-based magneto-caloric devices, as predicted by Brown in his seminal work on magneto-caloric refrigerators from $1976,{ }^{117}$ or by employing multilayered refrigerator designs. ${ }^{118,119}$ Magneto-caloric systems might also be up for a surprise at the somewhat neglected micro- and nanoscale; ${ }^{120}$ however considering the origin of the magneto-caloric effect, it is unlikely that higher cooling powers and larger temperature differences can be achieved for nanostructures. With more and more research groups joining the field of spin caloritronics, new effects might be discovered and existing ones considerably improved. ${ }^{106}$ By employing a series of nanoscale emitters in aggregate, forming a Spindt array, ${ }^{121}$ field-emission cooling powers might be sufficiently increased $^{111,122}$ and, perhaps, might be further improved in combination with near-field radiative cooling. ${ }^{123}$ The field of cooling solids by laser irradiation is quickly growing and possible materials for larger efficiency, cooling power, and $\Delta T$ and entirely new approaches, e.g., based on superradiance, are already under investigation. ${ }^{124,125}$ A combination of all (or some) of the above four cooling methods could be a suitable option but poses a serious design challenge in 
an HDD or a similar device. Transferring the waste heat to the recording media might also open new opportunities for HAMR or conventional PMR. ${ }^{109}$

\section{REVIEW OF HIGH-MOMENT MAGNETIC MATERIALS}

This review is ordered by material classes and centered around the improvement of the magnetic moments of magnetic TMs and RE metals in alloys, compounds, or at various interfaces with NMs and near-ferromagnets (such as Pd, $\mathrm{Pt}, \mathrm{Rh}$, and $\mathrm{Sc}$ ), SCs or stabilised by interstitial elements (IEs). First, the TMs (particularly $\mathrm{Fe}, \mathrm{Ni}$, and $\mathrm{Co}$ ) are discussed, followed by heavy REs (particularly Gd, Tb, Dy, and Ho) and eventually both material classes are looked at in combined TM-RE systems.

\section{A. Inter-TM alloys of $\mathrm{Fe}, \mathrm{Ni}$, and $\mathrm{Co}$ : The FeCo champion}

As early as 1912, Weiss investigated the intrinsic magnetic properties of the $\mathrm{FeNi}, \mathrm{NiCo}$, and $\mathrm{CoFe}$ binary alloy systems and identified the outstandingly large saturation magnetisation of the $\mathrm{Fe}_{2} \mathrm{Co}$ composition, which was found to be about $10 \%$ larger than that of pure Fe. ${ }^{126}$ In 1929 , a first patent was filed for the industrial-scale fabrication of the equiatomic FeCo alloy-a composition being particularly soft while still maintaining a remarkably large magnetic moment at room temperature. ${ }^{127} \mathrm{~A}$ few years later this rather brittle compound was stabilised by adding small quantities of $\mathrm{V}$, resulting in $(\mathrm{FeCo})_{98} \mathrm{~V}_{2}$ known as permendur, which triggered its vast industrial use ${ }^{128}$ (note, the term "permendur" is nowadays used for high-moment FeCo alloys in general). For its unchallenged high magnetic saturation at room temperature, FeCo alloys are the present day materials of choice for write pole tips in magnetic recording devices. ${ }^{3,129,130}$ The highest saturation magnetisation of all the $\mathrm{TM}$ alloys at room temperature of $\mu_{0} M_{\mathrm{S}}=(2.4 \pm 0.1) \mathrm{T}$ was achieved for $\mathrm{Fe}_{\mathrm{X}} \mathrm{Co}_{100-\mathrm{X}}$ alloys with $X=60-80$, as shown in Fig. 5(a), with most studies limiting the alloy range to $X=65-70$ (Refs. 130-132) and stating a respective saturation magnetisation of $\mu_{0} M_{\mathrm{S}}=2.45 \mathrm{~T} .^{4,35,133}$ This value is commonly referred to as the "Slater-Pauling limit,"134 since it is the maximum in the Slater-Pauling plot, which summarises the experimental results of magnetic moments of known TM alloys, as depicted in Fig. 5(b).

The Slater-Pauling curve is closely linked to the rigidband model, which is a well-known theory based on Hund's rule to predict the magnetic moment and resulting saturation magnetisation of a $3 d \mathrm{TM}$, assuming a fixed, spin-split density of states. ${ }^{135}$ This "density-of-state jug" (term introduced by $\mathrm{Coey}^{35}$ ) is filled up with the necessary electrons, which (ignoring the small $4 s$-band contribution and only taking into account the number of $3 d$ electrons $N_{3 d}$ ) gives an average magnetic moment $\bar{m}$ per atom for any strong ferromagnet of $\bar{m} \approx\left(10-N_{3 d}\right) \mu_{\mathrm{B}}$. This corresponds to the slope of the right-hand side of the Slater-Pauling curve in Fig. 5(b). In conclusion, a single $\mathrm{Fe}$ atom could theoretically have a moment of $4 \mu_{\mathrm{B}} /$ atom and Mn even $5 \mu_{\mathrm{B}} /$ atom. However, one finds that in the bulk, the BCC crystal structure of Fe does only lead to about $m_{\mathrm{Fe}}^{\mathrm{WM}} \approx 2.2 \mu_{\mathrm{B}}$ /atom, the equivalent of
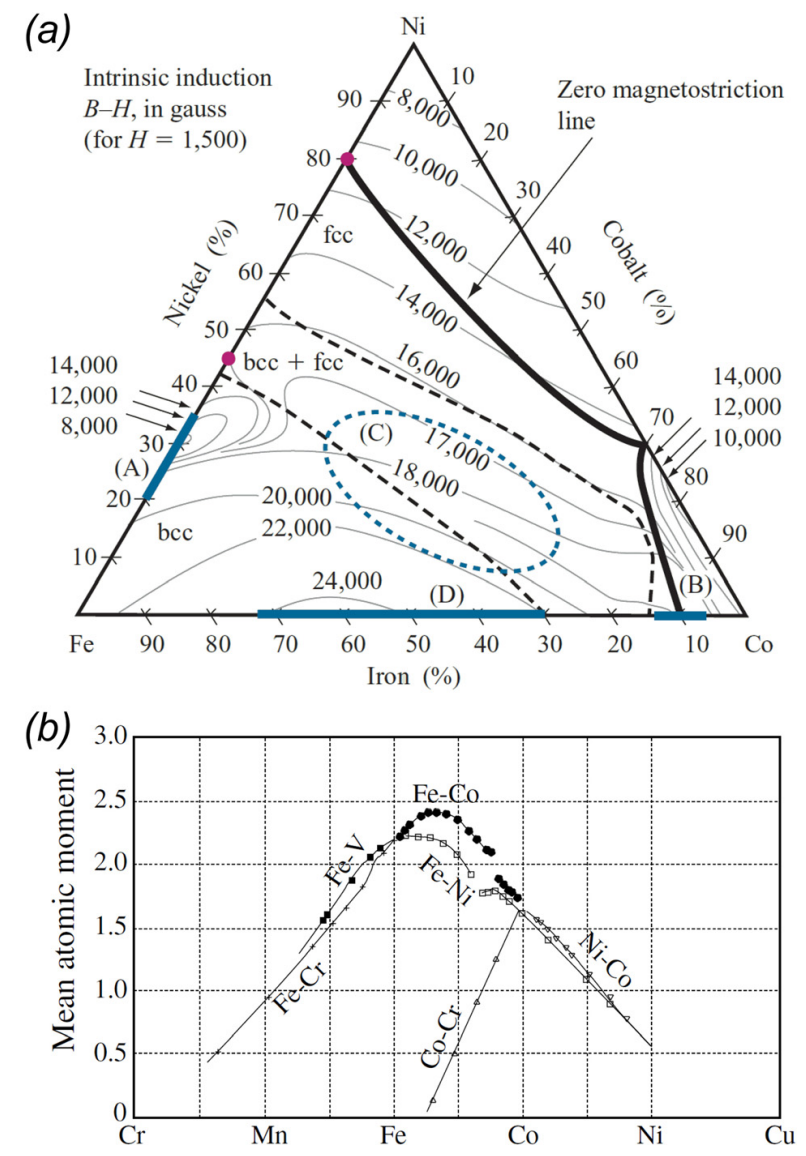

FIG. 5. (a) The magnetic phase diagram of the FeCoNi ternary alloy system. Four compositions are particularly relevant for pole-piece application: (A) Fe-rich FeNi alloys, (B) Co-rich CoFe alloys, (C) CoNiFe alloys, and (D) high-moment FeCo alloys. (b) Slater-Pauling curve which is a plot of magnetic moment measurements of several TM alloys, where the maximum saturation magnetisation was found for $\mathrm{Fe}_{65-70} \mathrm{Co}_{35-30}$ alloys with $\mu_{0} M_{\mathrm{S}}=2.45 \mathrm{~T}$ - the Slater-Pauling limit. Graph (a) is reproduced with permission from Cooper et al., IBM J. Res. Dev. 49, 103 (2005). Copyright 2005 International Business Machines Corporation, ${ }^{3}$ and is an adaptation of an original figure in Ferromagnetism by Bozorth. ${ }^{138}$ Graph (b) is reprinted with permission from Sourmail, Prog. Mater. Sci. 50, 816 (2005). Copyright 2005 Elsevier. ${ }^{128}$

$\mu_{0} M_{\mathrm{S}}=2.15 \mathrm{~T}^{35}$ This is due to Fe being a weak ferromagnet, rather than a strong ferromagnet such as $\mathrm{Co}$ and $\mathrm{Ni}$. The spinup part of the $3 d$ band of a strong ferromagnet is completely filled and pushed below the Fermi level, whereas in a weak ferromagnet it is only partly filled, leading to a reduced magnetic moment. A more realistic prediction of the $\mathrm{Fe}$ moment in a crystal, assuming a strong ferromagnet, can be obtained by following the slope of the right-hand side of the Slater-Pauling curve in Fig. 5(b), giving $m_{\mathrm{Fe}}^{\mathrm{SM}} \approx 2.7 \mu_{\mathrm{B}}$ /atom. An example of Fe being a strong ferromagnet is the $\mathrm{Fe}_{4} \mathrm{~N}$ compound, where interstitial $\mathrm{N}_{2}$ stabilises an FCC Fe sublattice. However, the overall saturation magnetisation is only $\mu_{0} M_{\mathrm{S}}=1.89 \mathrm{~T}$, since not all $\mathrm{Fe}$ atoms have a high atomic moment and $\mathrm{N}$ is negatively polarised (see Fig. 3).

More detailed theoretical studies of FeCo alloys and ordered structures (in BCC crystals) reproduce the SlaterPauling curve rather well-which clearly demonstrates the accuracy of the first-principle based methods-and also lend some additional insight in the magnetic structure of these materials (see Fig. 6). The calculations reproduce the initial 


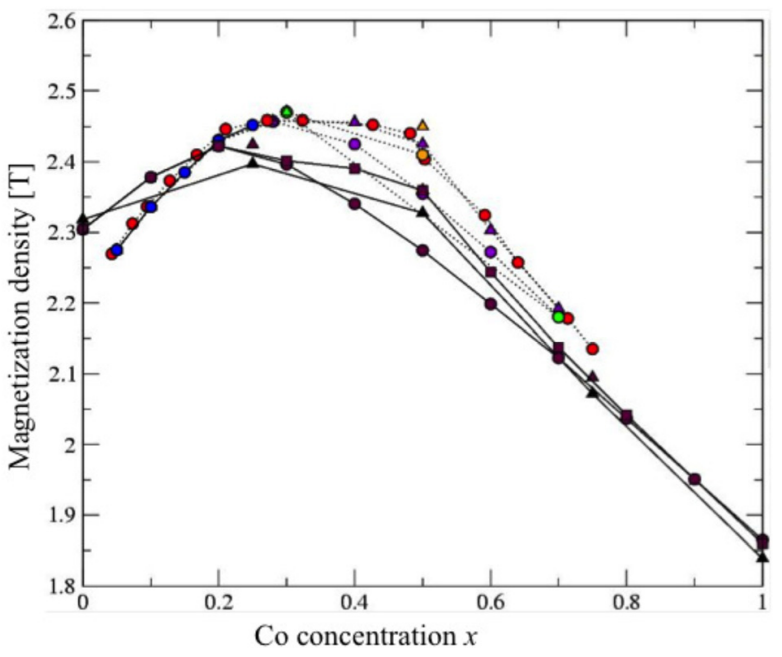

FIG. 6. Experimental and theoretical magnetic moments of FeCo alloys (BCC). Ordered alloys are represented by triangles, and disordered alloys by circles. Purple circles and triangles denote experimental data from Bardos $;{ }^{136}$ red circles are experimental data from Ref. 137; green circles and triangles are experimental data from Bozorth; ${ }^{138}$ blue circles denote experimental data from Parette and Mirebeau; ${ }^{139}$ black symbols are from DFT modeling ${ }^{140}$ for ordered (triangles) and different disorder modeling (circles and squares).

increase in the saturation moment of $\mathrm{Fe}$ as $\mathrm{Co}$ is substituted as well as the calculated maximum in the moment before a moment of $1.8 \mu_{B} /$ atom for BCC Co. The net magnetic moments in the calculations are composed of smaller orbital magnetic moments $\left(0.05-0.1 \mu_{B} /\right.$ atom $)$ and a large spin moment, in agreement with observations. The calculations also reproduce the observation that ordered alloys have a larger saturation moment compared to the disordered system $\left(0.05-0.07 \mu_{B} /\right.$ atom $)$. The reason for this is that Fe atoms surrounded by $\mathrm{Co}$ atoms in the shell of nearest neighbours have larger moments than $\mathrm{Fe}$ atoms which have some $\mathrm{Fe}$ and some Co atoms in this shell. A system with completely ordered FeCo has all $\mathrm{Fe}$ atoms in a favourable, high-moment geometry. Any level of disorder reduces the fraction of highmoment $\mathrm{Fe}$ atoms. In the calculations, it is found (not shown) that Co for all concentrations and degrees of disorder has a moment of $1.8 \mu_{B}$ /atom.

The Curie point of high-moment FeCo alloys was calculated to be $T_{\mathrm{C}} \approx 1370 \mathrm{~K} ;{ }^{141}$ however, they were found to undergo a phase transition at $T_{\mathrm{PT}} \approx 1250 \mathrm{~K},{ }^{142}$ which increases the effective Curie point to $T_{\mathrm{C}} \approx 1500 \mathrm{~K}$ [magnetisation vs. temperature profile see Fig. 7(a)]. At room temperature, high $\mathrm{Fe}$-content $\mathrm{FeCo}$ alloys were found to primarily order $\mathrm{BCC}$, in the $\mathrm{CsCl}$-like $\mathrm{B} 2\left(\alpha^{\prime}\right)$ phase with a lattice parameter of $a=2.86 \AA{ }^{128}$ For $T>1250 \mathrm{~K}$, it turns into the FCC-based $\gamma$ phase. ${ }^{141}$ Thin films grow preferably in the BCC $\left(\begin{array}{lll}1 & 1 & 0\end{array}\right)$ texture at room temperature. ${ }^{131}$ Although inferior in terms of saturation magnetisation, the $\mathrm{Fe}_{50} \mathrm{Co}_{50}$ composition is softer and benefits from a much higher initial permeability as depicted in Fig. 7(b). Permeablilty can also be increased by adding $\mathrm{Cu}$ or $\mathrm{Ni}$ to $\mathrm{FeCo}$, whereas pure $\mathrm{Fe}_{\mathrm{X}} \mathrm{Ni}_{100-\mathrm{X}}$ alloys can be synthesised to have huge permeability and negligible magnetostriction [see bold line in Fig. 5(a)], which made it the material system of choice for
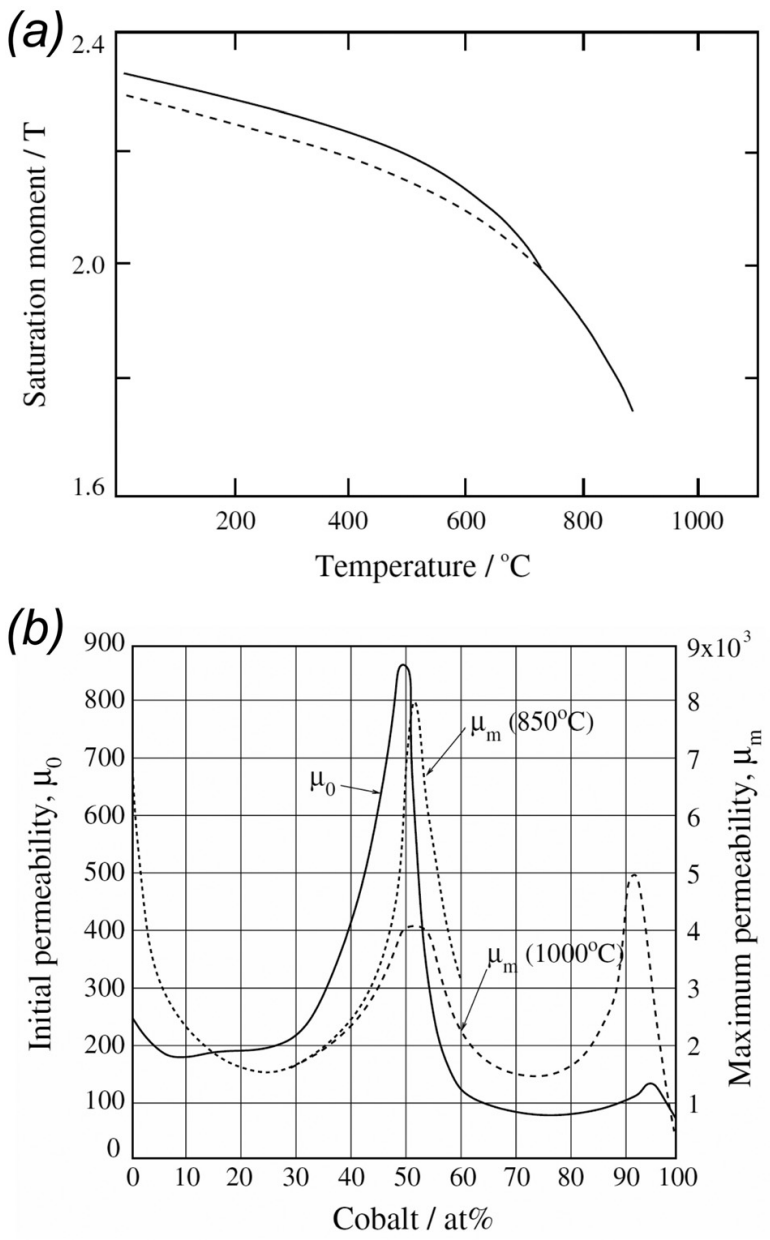

FIG. 7. (a) Magnetisation vs. temperature profile for FeCo, showing a phase transition at $T_{P T} \approx 1250 \mathrm{~K}$ and indicating an effective Curie point of $T_{\mathrm{C}} \approx 1500 \mathrm{~K}$. (b) The advantage of $\mathrm{Fe}_{50} \mathrm{Co}_{50}$ over $\mathrm{Fe}_{65-70} \mathrm{Co}_{35-30}$ : much higher initial permeability (given temperatures are annealing values). Graphs (a) and (b) are reprinted with permission from Sourmail, Prog. Mater. Sci. 50, 816 (2005). Copyright 2005 Elsevier. ${ }^{128}$

write pole tips prior to $\mathrm{FeCo},{ }^{1}$ particularly $\mathrm{Ni}_{78-80} \mathrm{Fe}_{20-22}$ permalloy and $\mathrm{Ni}_{45} \mathrm{Fe}_{55} \cdot{ }^{3}$ With the advent of HAMR and the requirement of ultra-fast switching which has to be synchronised with the near-field heating, NiFe (as in $\mathrm{NiFe} /$ FeCo laminates ${ }^{143}$ ) might again be of interest in the near future, for writing in the Hopkinson peak of the recording media, i.e., when less field strength but good timing is required.

Adding Mn to FM TMs does not lead to write-pole relevant magnetic properties superior to pure FeCo. Noticably, there are some studies on $\mathrm{CoMnP}^{144}$ and $\mathrm{CoNiMnP}^{145,146}$ which indicate saturation magnetisations of $\mu_{0} M_{\mathrm{S}}>2 \mathrm{~T}$ (see also Guan and Nelson ${ }^{147}$ who reported much lower $B_{\mathrm{S}}$ values), but due to large crystal anisotropy (with the easy axis perpendicular to the plane of the textured thin films) neither softness nor initial permeability is a match for FeCo. Due to its AFM coupling to $\mathrm{Fe}, \mathrm{Ni}$, or $\mathrm{Co}, \mathrm{Cr}$ is also not a useful additive when seeking high saturation magnetisation alloys or laminates. ${ }^{148}$ However, $\mathrm{Cr}$ is a common seed material for FeCo write pole tips due to a good crystal match, a momentum conservation of adjacent TMs at interfaces, and the 
formation of a nanogranular soft FeCo phase. ${ }^{149,150}$ Further opportunities which arise from the notorious AFM coupling of $\mathrm{Cr}$ (and $\mathrm{Mn}$ ) to TMs - and to RE metals alike-are discussed in Section II G 3.

To date, no other bulk material has been found to reliably exhibit higher saturation magnetisation at room temperature than $\mathrm{Fe}_{2} \mathrm{Co}^{35}$ Research is still ongoing to push the saturation of FeCo alloys beyond the present day limit, by creating pure single crystals of defined orientation ${ }^{151}$ which do not suffer from moment losses due to grain boundaries, ${ }^{152}$ or multi-layered systems. First-principle based modeling of ordered Fe-Co multilayers shows that the spin moment of $\mathrm{Fe}$ increases near the $\mathrm{Fe}-\mathrm{Co}$ interface, while the Co spin moment decreases less, leading to a net increase in spin moment (see Fig. 8). There are also much smaller changes in the orbital magnetic moments of the atoms near the interface. Therefore, such multilayer structures can in principle provide a higher net magnetisation density than alloys.

Nanodimensional systems with corrosion protection (i.e., diffusion barries) are of particular interest for future recording devices in the light of further device miniaturization. ${ }^{153-155}$ There is also research aimed at improving the magnetic softness with a focus on investigating highly (nano-) granular or even amorphous FeCo. ${ }^{156,157}$ The crystalline ordering was also found to be influenced by (fieldassisted) annealing, ${ }^{132,158}$ by the formation of various (soft) compounds such as $\mathrm{FeCoN}^{159}$ (see also Section IIE), $\mathrm{FeCoAlO},{ }^{160} \mathrm{FeCoB},{ }^{161}$ and by using alternative seed layer materials such as $\mathrm{NiFe}, \mathrm{AlO}, \mathrm{Ru}$, or $\mathrm{Cu}{ }^{130,131}$ A general trend is the formation of a soft BCC FeCo phase when deposited on an FCC seed layer such as $\mathrm{Cu} .{ }^{162}$ Some of those seed layers were also found to counteract high magnetostriction and undesired anisotropies of pure $\mathrm{FeCo}$, particularly in laminates. ${ }^{163,164}$ It was found that FeCo films fabricated by oblique sputtering (seed and magnetic layer are deposited at a different angle between target and gun) benefit from favourable anisotropies for write poles in PMR devices. ${ }^{74,132}$

It is worth noting that, although literature on thin films of FeCo commonly cites the bulk saturation values, actual measurements on thin films of $\mathrm{Fe}_{65-70} \mathrm{Co}_{35-30}$ show a

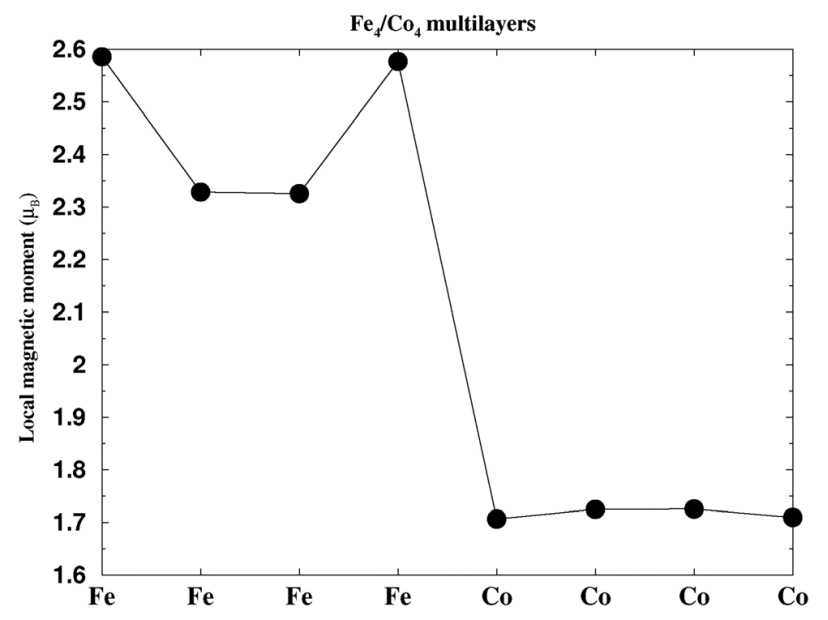

FIG. 8. Modeled spin magnetic moment on $\mathrm{Fe}$ and $\mathrm{Co}$ atoms in a $\mathrm{BCC}$ $\mathrm{Fe}_{4} \mathrm{Co}_{4}$ multilayer structure. ${ }^{140}$ reduced saturation magnetisation of $\mu_{0} M_{\mathrm{S}}<2.4 \mathrm{~T}^{4,131,162,165}$ There is yet no satisfying answer for why there is a moment drop in these thin films compared to the bulk, but finite-size effects are believed to play an important role.

\section{B. TM/NM interfaces, superlattices, and TM-NM alloys}

Artificially layered systems of a magnetic TM and NM were shown to exhibit a significant moment increase in the TM film. These systems benefit from a good lattice match reducing interfacial strain, as in $\mathrm{Fe}$ and $\mathrm{Au},{ }^{166}$ chemical stability and protection due to the NM, and only weak magnetic TM-NM coupling. ${ }^{167}$ Studies so far indicate that moment increase is achieved either by stabilising metastable crystal phases with a different spin state due to a larger atomic radius ${ }^{168,169}$ or by a transition of the magnetic layer to a 2dimensional (2D) magnetic system with changes in electronic and hence magnetic structure. ${ }^{170,171}$

\section{Fe atoms at $N M(N M=A u, A g, P d)$ interfaces and in nanolaminates}

In a series of theoretical studies on the electronic and magnetic properties of ultrathin Fe films ${ }^{172,173}$ it was shown that for an isolated ML of Fe the magnetic moment per atom can increase up to $m=3 \mu_{B} /$ atom (corresponds to $\mu_{0} M_{\mathrm{S}} \approx 3 \mathrm{~T}$ ). Similar calculations were done for the $\mathrm{Fe} / \mathrm{Ag}$ system yielding similar results of a moment increase. ${ }^{174,175}$ Subsequent detailed calculations by Crampin ${ }^{170}[\mathrm{Fe}$ on $\mathrm{Au}$ (001)], MacLaren et al. ${ }^{166}$ (Fe/Au superlattices and interfaces), and Stoeffler ${ }^{167}(\mathrm{Ag} / \mathrm{Fe} / \mathrm{X}$, with $\mathrm{X}=\mathrm{Pd}, \mathrm{Cu}, \mathrm{Ag}$, and $\mathrm{Au}$ ) confirmed the moment increase [see Fig. 9(a)] and explained it in terms of changes in the $3 d$-band structure of the $\mathrm{Fe}$ atoms as a result of the transition to a $2 \mathrm{D}$ magnetic system in ultrathin films.

Experimental proof of an improved saturation magnetisation of ultrathin Fe films was found by Wooten et al. ${ }^{171}$ who observed a magnetic moment of $m=2.6 \mu_{B} / \mathrm{Fe}$ atom for $3 \mathrm{ML} \mathrm{Fe}$ at an $\mathrm{Fe} / \mathrm{Ag}(100)$ interface, by Pan et al. ${ }^{176}$ who also reported $m=2.6 \mu_{B} / \mathrm{Fe}$ atom for $\mathrm{Fe} / \mathrm{Au}$ nanomultilayers, by Muehlbauer et al. ${ }^{168}$ who reported $m=2.7 \mu_{B} / \mathrm{Fe}$ atom for ultrathin FCC Fe films in Fe/Pd laminates, and in a recent study by Jal et al. ${ }^{177}$ who recorded a magnetisation depth profile [via X-ray resonant magnetic reflectivity (XRMR), see Fig. 10(a)] of an ultrathin Fe film grown on a vicinal (i.e., stepped) $\operatorname{Ag}(001)$ surface and capped by Au. They found a particularly high Fe moment of $m=2.8 \mu_{B} / \mathrm{Fe}$ atom for the second Fe ML as shown in Fig. 10. Theoretical and experimental studies agree on there is little to no polarisation in the NM, as shown in Fig. 9(b), except for the case of weakly polarised $\mathrm{Pd}$ whose induced moment is still very small compared to the Fe. ${ }^{168,178}$

There is very limited data on the magnetic phase transition, but in line with theoretical predictions, ${ }^{170}$ magnetisation vs. temperature measurements show a disproportionally large moment drop (compared to bulk Fe) with increasing temperature for $\mathrm{Fe} / \mathrm{Pd}$ laminates, ${ }^{168,179}$ resulting in effectively no moment gain at room temperature. Another study indicates a Curie point of $T_{\mathrm{C}} \approx 300 \mathrm{~K}$ for $3 \mathrm{ML}$ of Fe grown on $\mathrm{Ag}(100)$ substrates $^{171}$ and a $50 \%$ drop in $T_{\mathrm{C}}$ was reported 

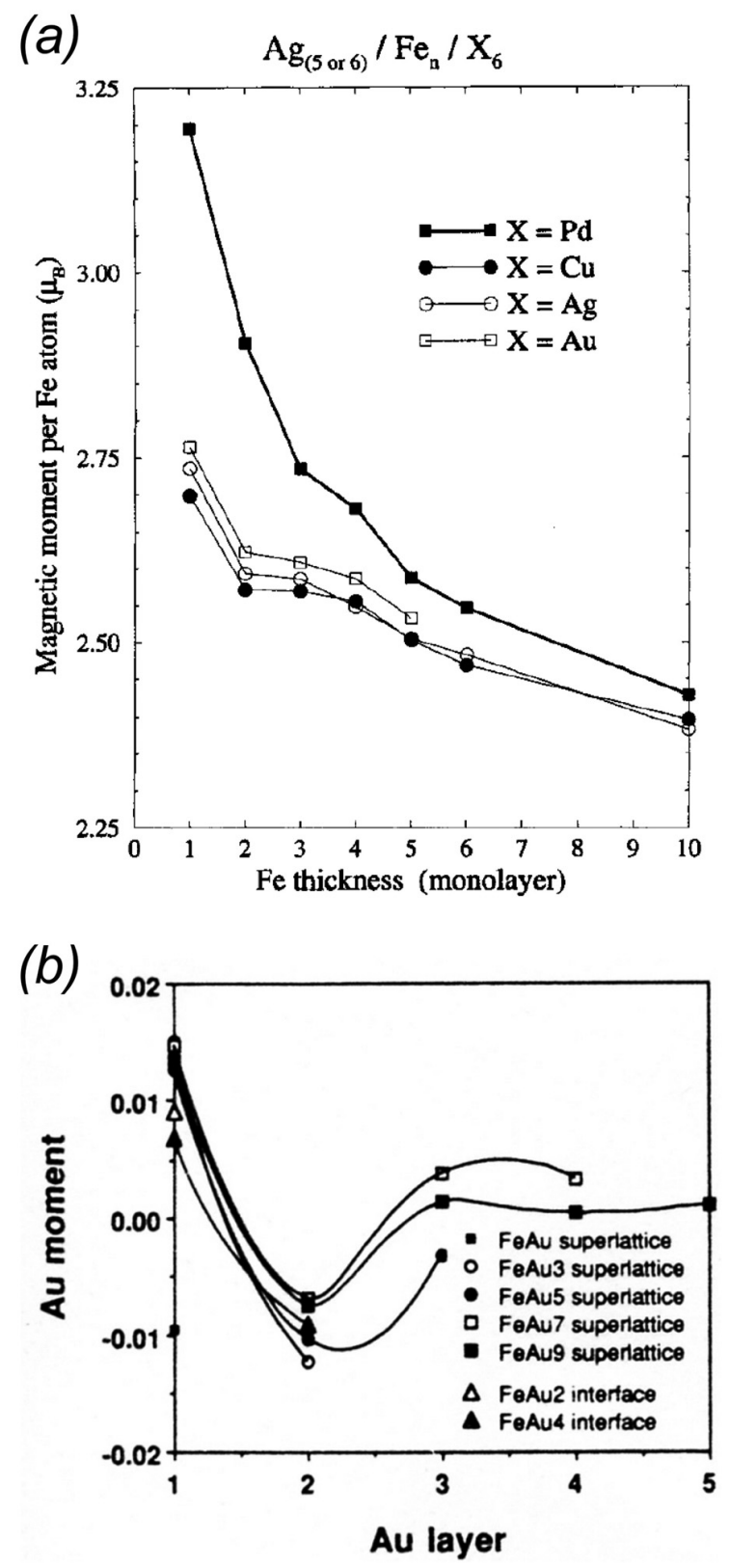

FIG. 9. (a) Calculations of the magnetic moment of Fe which strongly increases in MLs at NM interfaces, with a maximum for $\mathrm{NM}=\mathrm{Pd}$. (b) Induced magnetic moment in Au MLs in proximity to Fe. Graph (a) is reprinted with permission from Stoeffler, J. Magn. Magn. Mater. 165, 62 (1997). Copyright 1997 from Elsevier. ${ }^{167}$ Graph (b) is reprinted with permission from J. Appl. Phys. 67, 5406 (1990). Copyright 1990 AIP Publishing LLC. ${ }^{166}$

by Bader et al. ${ }^{180}$ for an ML of Fe grown on Au. A comprehensive overview of measured magnetic moments and Curie temperatures of $\mathrm{Fe}, \mathrm{Ni}$, and $\mathrm{Co}$ is given by Vaz et al. ${ }^{181}$ and lists the ultrathin film or interface system as well as the measuring technique in use. This brief summary of reports on magnetic moment values in ultrathin films also shows the large degree of ambiguity, with different experimentalists reporting increase or decrease for very similar or even identical systems. The overall theme though is a moment increase at cryogenic temperatures for nearly all ultrathin films of $3 d$ (a)
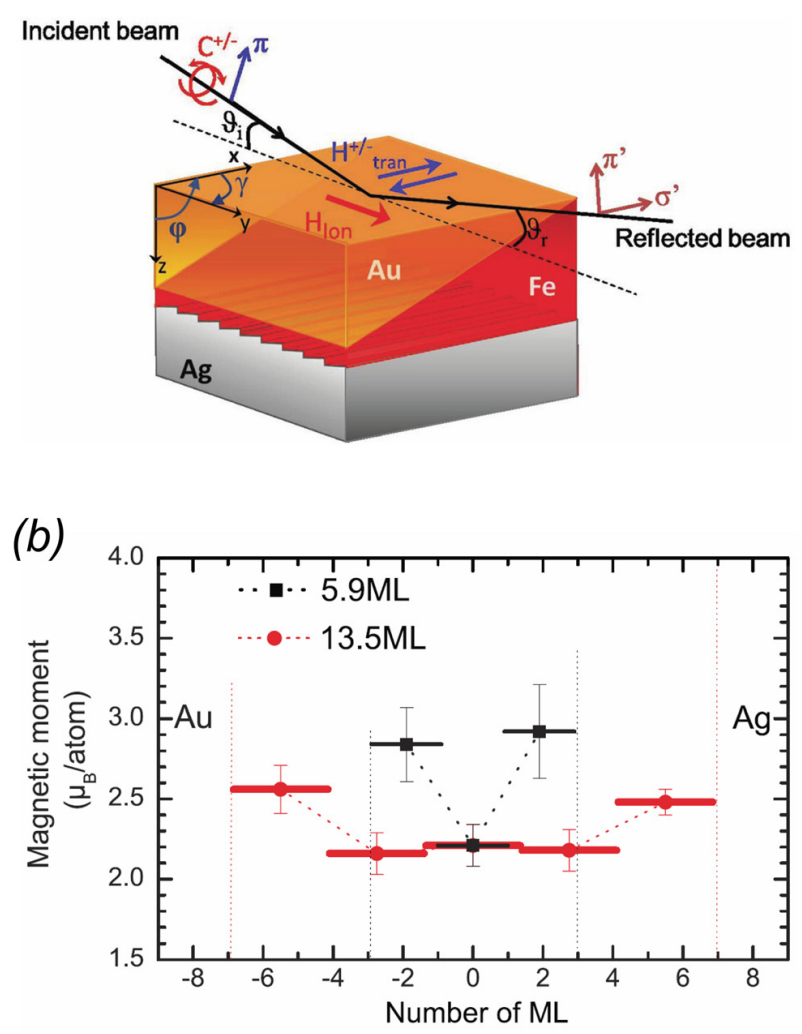

FIG. 10. (a) Stack composition and XRMR measurement setup as used by Jal et al. for recording the magnetisation depth profile. (b) They show a strongly improved Fe moment in the second ML for films grown on a vicinal Ag surface, capped by Au. Figures reprinted with permission from Jal et al., Phys. Rev. B 87, 224418 (2013). Copyright 2013 the American Physical Society. ${ }^{177}$

TMs (in combination with NMs), with Fe layers exceeding the saturation magnetisation of bulk FeCo alloys.

\section{Fe-NM (NM = Au, Ag, Pd, and Rh) and FeSc alloys}

Similar to the effect of increasing the magnetic moment of $\mathrm{Fe}$ atoms at interfaces of $\mathrm{NMs}, \mathrm{Fe}$ moments increase in disordered NM alloys, i.e., the $\mathrm{Fe}$ moment per atom increases when diluted in a NM matrix. This effect was also attributed to the narrowing of the $3 d$ band and it was found that the $\mathrm{Fe}$ moment gradually increases with dilution and reaches $m=3.0 \mu_{B}$ /atom when being heavily diluted in a $\mathrm{Au}$ matrix [see Fig. 11(a)]. ${ }^{182}$ However, the overall moment of the entire alloy is well below bulk Fe for any composition ${ }^{183}$ as shown in Fig. 11(b). Similar behaviour was found for $\mathrm{FeAuB}$ alloys of various composition, ${ }^{184}$ where the $\mathrm{Fe}$ moment levelled at $m=2.6 \mu_{B} /$ atom, and Curie temperatures were reported to be $630 \mathrm{~K} \leq T_{\mathrm{C}} \leq 730 \mathrm{~K}$, i.e., well above room temperature. Increasing moments were also found for other TMs, such as $\mathrm{Ni}$ and $\mathrm{Co},{ }^{185}$ and other NMs such as $\mathrm{Ag},{ }^{186} \mathrm{Pd},{ }^{167,187,188}$ and $\mathrm{Rh} .{ }^{189}$

Altogether, no Fe-NM alloy has been reported to exceed bulk Fe magnetisation, not to mention FeCo. No moment enhancement was observed for Fe atoms in proximity to Sc, which is also regarded a near-ferromagnet such as $\mathrm{Pd}$ for it 


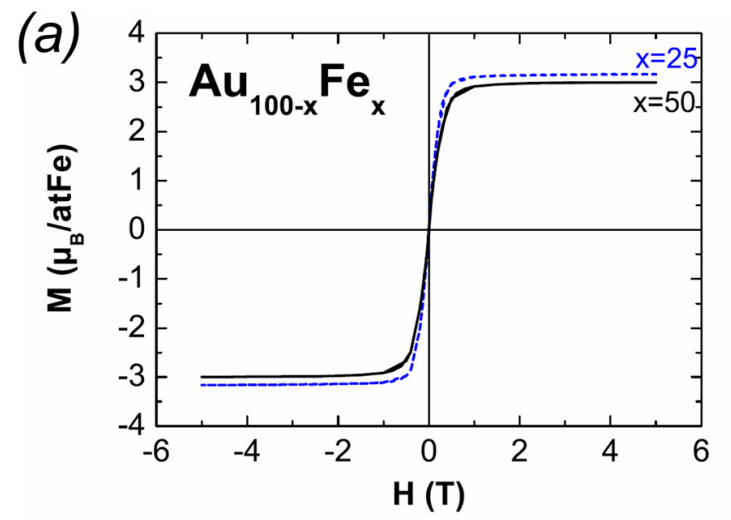

(b)

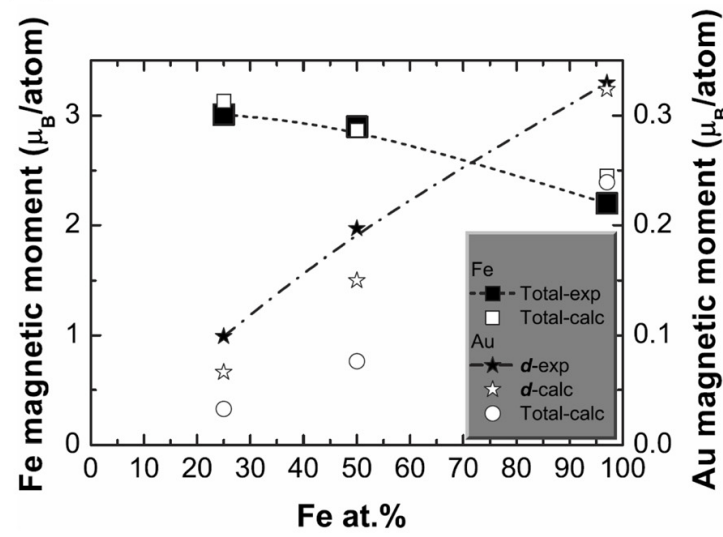

FIG. 11. (a) Hysteresis loop of two FeAu alloys measured at $T=4 \mathrm{~K}$ and (b) magnetic moments of $\mathrm{Fe}$ and $\mathrm{Au}$ in $\mathrm{FeAu}$ alloys of various composition as reported by Wilhelm and coworkers. Graphs (a) and (b) are reprinted with permission from Wilhelm et al., Phys. Rev. B 77, 224414 (2008). Copyright 2008 the American Physical Society. ${ }^{182}$

nearly fulfills the Stoner criterion, and alloys of higher Sc content even loose their simple FM-PM ordering. ${ }^{190}$

\section{FeRh}

Although TM-NM alloys do not possess saturation magnetisations superior to $\mathrm{FeCo}, \mathrm{FeRh}$ is worth some extra attention. The material shows a most peculiar transition from AFM to FM with increasing temperature around room temperature. This transition is strictly dependent on the composition of the $\mathrm{Fe}_{1-X} \mathrm{Rh}_{\mathrm{X}}$ alloy, existing only for $X \approx 0.5-0.6$ (Ref. 191) [see Fig. 12(a)], and also on the alloy microstructure $^{192}$ [see Fig. 12(b)]. The most common explanation for the phenomena links the magnetic phase change to a change in the crystal structure of BCC-ordering FeRh: an abrupt isotropic increase of the lattice spacing coincides with the magnetic transition temperature. ${ }^{193}$ Both intrinsic dependencies, composition and microstructure, are also ultimately connected to the lattice spacing.

However unique the magnetisation behaviour of FeRh might be, a saturation magnetisation of $\mu_{0} M_{\mathrm{S}} \approx 1.6 \mathrm{~T}$ at best (just above the AFM-FM transition for the equiatomic alloy ${ }^{194}$ ) is far too low to be useful in write head pole pieces. Nevertheless, FeRh might be of importance for HAMR in $\mathrm{FePt} / \mathrm{FeRh}$ exchange-spring coupled media, where the FeRh (a)
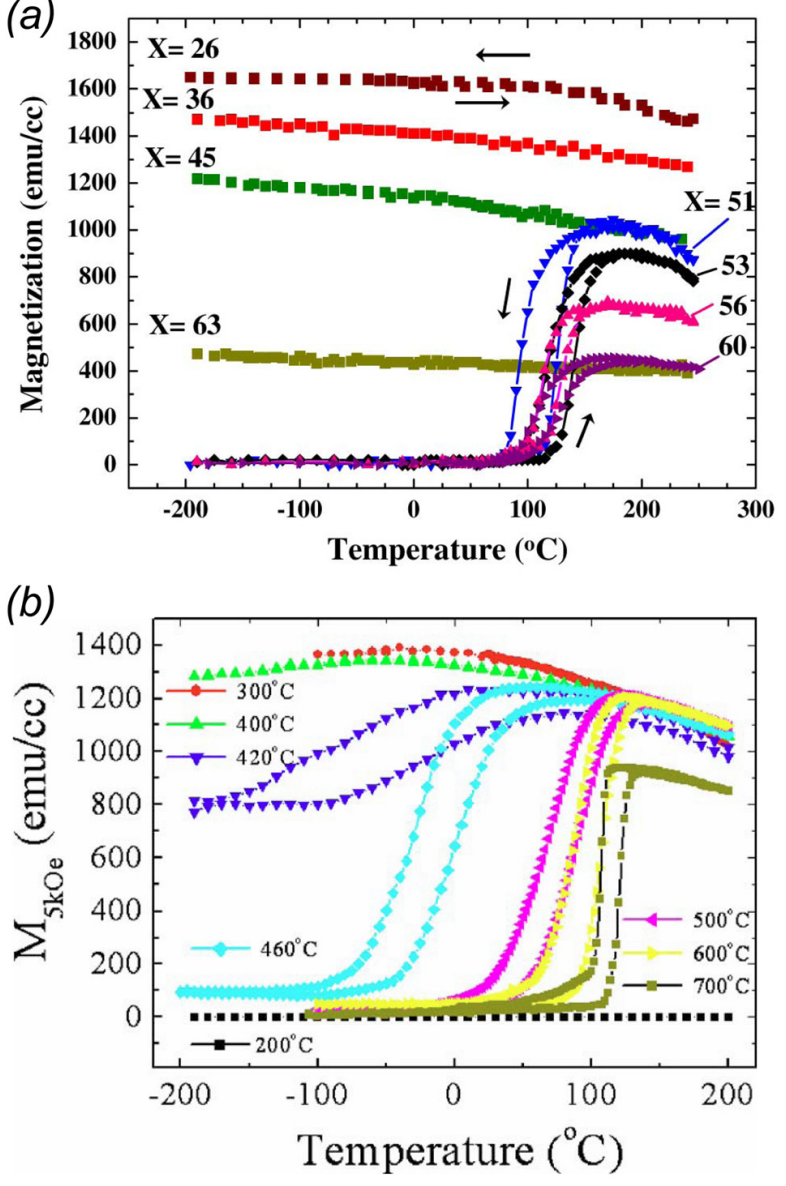

FIG. 12. Magnetisation dependences of $\mathrm{Fe}_{1-X} \mathrm{Rh}_{X}$ thin films: (a) The peculiar AFM-FM transition upon heating only occurs for compositions of $X \approx 0.5-0.6$; and (b) is dependent on the crystallisation of the alloy-such as resulting from annealing (respective annealing temperatures are in the inset; $X=0.51$ ). Both dependencies are ultimately linked to changes in the interatomic spacing. Graph (a) is reprinted with permission from Inoue et al., IEEE Trans. Magn. 44, 2875 (2008). Copyright 2008 IEEE. ${ }^{191}$ Graph (b) is reprinted with permission from J. Appl. Phys. 103, 07F501 (2008). Copyright 2008 AIP Publishing LLC. ${ }^{192}$

effectively reduces the critical temperature and, hence, eases the write field strength requirement ${ }^{195}$ — while keeping the current single-pole design of PMR systems. Concerns were raised regarding device integration, since high-coercivity $\mathrm{L}_{0}$-phase $\mathrm{FePt}$ recording medium requires nanogranular (nanocolumnar) growth, which has not been achieved for FePt-FeRh stacks so far. ${ }^{196}$

\section{FeCo/Pd laminates and FeCo:Pd alloys}

In a 2005 study on magnetron-sputtered $\mathrm{Fe}_{70} \mathrm{Co}_{30} / \mathrm{Pd}$ superlattices and $\mathrm{Fe}_{70} \mathrm{Co}_{30}$ :Pd alloys [stack composition see Fig. 13(a)], Noma et al. ${ }^{197}$ reported an effective saturation magnetisation of up to $\mu_{0} M_{S}^{F e C o}=2.57 \mathrm{~T}$ for FeCo layers sandwiched by $2 \mathrm{~nm} \mathrm{Pd}$ in the laminate at room temperature [see Fig. 13(b)], measured with a SQUID magnetometer. In a subsequent study a year later, ${ }^{4}$ they then presented an $\mathrm{Fe}_{70} \mathrm{Co}_{30}: \mathrm{Pd}$ alloy with a saturation of $\mu_{0} M_{\mathrm{S}}=2.49 \mathrm{~T}$ [see Fig. 14(a)] and an $\mathrm{Fe}_{70} \mathrm{Co}_{30} / \mathrm{Pd}$ multilayer system with an overall saturation magnetisation of $\mu_{0} M_{\mathrm{S}}=2.57 \mathrm{~T}$ for the entire stack [see Fig. 14(b)]—both would effectively crack 
(a)

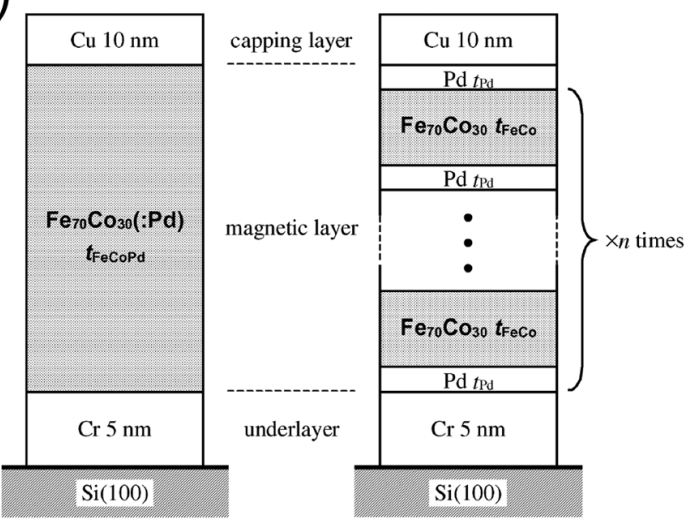

(b)

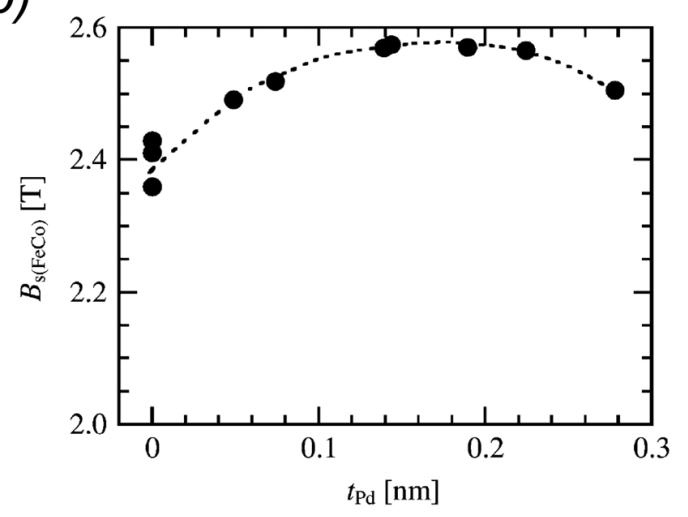

FIG. 13. (a) Stack setup under investigation by Noma and coworkers. ${ }^{4}$ (b) Improved saturation magnetisation of $\mathrm{FeCo}$ films in $\mathrm{FeCo} / \mathrm{Pd}$ laminates as reported by Noma et al.: ${ }^{197}$ a maximum was achieved for a Pd spacer layer thickness of about $t_{\mathrm{Pd}}=0.14 \mathrm{~nm}$ for $t_{\mathrm{FeCo}}=1.7 \mathrm{~nm}$ thick $\mathrm{Fe}_{70} \mathrm{Co}_{30}$ layers. Graph (a) is reprinted with permission from Noma, IEEE Trans. Magn. 42, 140 (2006). Copyright 2006 IEEE. Graph (b) is reprinted with permission from Noma et al., IEEE Trans. Magn. 41, 2920 (2005). Copyright 2005 IEEE.

the Slater-Pauling limit. The stacks under investigation were $30 \mathrm{~nm}$ and $40 \mathrm{~nm}$ thick in total. The golden ratio for the $\mathrm{Fe}_{70} \mathrm{Co}_{30}$ :Pd alloys was found to be 1.04 at. \% Pd [see Fig. 14(a)] and the highest moment laminate was comprised of $t_{\mathrm{FeCo}}=5.1 \mathrm{~nm}$ thick $\mathrm{Fe}_{70} \mathrm{Co}_{30}$ layers [see Fig. 14(b)] separated by $t_{\mathrm{Pd}}=0.14 \mathrm{~nm}$ thick Pd spacers. Their pure $\mathrm{Fe}_{70} \mathrm{Co}_{30}$ reference layer yielded a saturation of $\mu_{0} M_{\mathrm{S}}=2.38 \mathrm{~T}$ and they assumed a magnetically dead layer of $1.5 \mathrm{~nm}$ thickness for the entire stack, ${ }^{197}$ which was subtracted from the volume of the magnetic material of the stack, thus effectively increasing the saturation magnetisation.

Considering the large number of studies that report improved moments for $\mathrm{Fe}$ atoms in $\mathrm{Fe} / \mathrm{Pd}$ laminates (see Section II B 1), the results by Noma et al. do not seem unrealistic, which is supported by an XMCD analysis of the above high-moment laminates by Noma et al. ${ }^{198}$ (stating a maximum Fe moment of $2.8 \mu_{\mathrm{B}}$ ). However, no subsequent study confirmed the moment increase for the entire stack, including the Pd spacer (see Walock et al. ${ }^{199}$ ). The assumption of magnetically dead layers by Noma et al. is somewhat unusual for NM-TM interfaces and by deducing it from the overall stack thickness a slightly higher saturation value is won. Putting it back into the calculation for the overall stack (a)

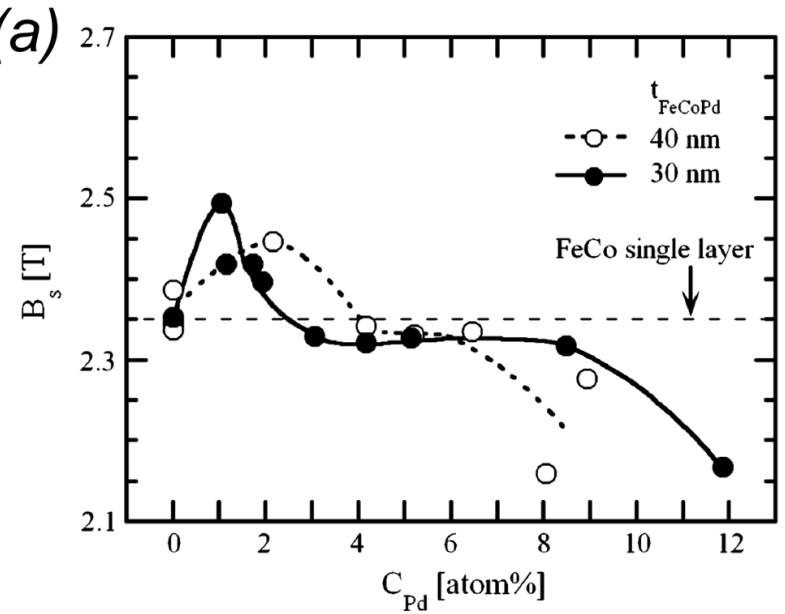

(b)

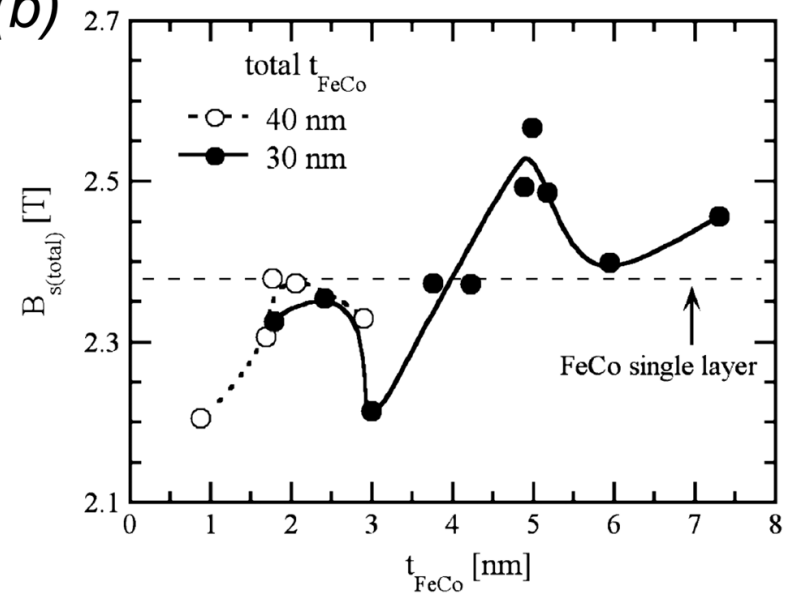

FIG. 14. Saturation magnetisations beyond the Slater-Pauling limit as reported by Noma et al., ${ }^{4}$ for thin films of (a) FeCo:Pd alloys and (b) $\mathrm{FeCo} / \mathrm{Pd} \mathrm{lami-}$ nates. A maximum saturation was found for the $\mathrm{Fe}_{70} \mathrm{Co}_{30}$ : $\mathrm{Pd}$ alloy for 1.04 at. $\%$ of $\mathrm{Pd}$; and for the laminate for $t_{\mathrm{FeCo}} 5.1 \mathrm{~nm}$ thick $\mathrm{Fe}_{70} \mathrm{Co}_{30}$ layers separated by $t_{\mathrm{Pd}}=0.14 \mathrm{~nm}$ Pd. Graphs (a) and (b) are reprinted with permission from Noma et al., IEEE Trans. Magn. 42, 140 (2006). Copyright 2006 IEEE.

thickness, one would get a saturation magnetisation of $\mu_{0} M_{\mathrm{S}}=2.45 \mathrm{~T}$ for the highest moment laminate, matching the Slater-Pauling limit. Due to the absence of error bars (or an uncertainty discussion), it is hard to assess the accuracy of the results, but the scatter of measured saturation values, such as in Figs. 14(a) and 14(b), would suggest at least $\Delta\left(\mu_{0} M_{\mathrm{S}}\right)=0.05 \mathrm{~T}$.

Although the concerns listed above question the absolute values of the saturation magnetisation in those films, the relative improvement of about $8 \%$ compared to the pure $\mathrm{FeCo}$ thin film reference sample seems possible and the $\mathrm{FeCo} / \mathrm{Pd}$ laminate might be a suitable candidate to at least reach the Slater-Pauling limit in thin films (at room temperature) due to the transition to a $2 \mathrm{D}$ magnetic system. ${ }^{200}$ Another study by Brankovic et al. ${ }^{56}$ where FeCoPd alloys were electrodeposited, and not plasma-sputtered, concluded that $\mathrm{Fe}_{61} \mathrm{Co}_{38} \mathrm{Pd}_{1}$ at least does not suffer from moment loss compared to high-moment $\mathrm{Fe}_{63} \mathrm{Co}_{37}$. The additional benefit of improved corrosion resistance was highlighted, which is a big advantage for pole tips in future HAMR systems. 


\section{FeCo epitaxially grown ultrathin films}

In a study on MBE-grown $\mathrm{Fe}_{\mathrm{X}} \mathrm{Co}_{1-\mathrm{X}}$ ultrathin films on (1 00 ) diamond substrates capped with Au, Ambrose et al. ${ }^{169}$ reported the fabrication and stabilisation of up to $2.5 \mathrm{~nm}$ thick FCC $\mathrm{Fe}_{70} \mathrm{Co}_{30}$ films [see Fig. 15(a)] with an outstanding saturation magnetisation of $\mu_{0} M_{\mathrm{S}}=(2.70 \pm 0.38) \mathrm{T}$ at room temperature [see Fig. 15(b)].

Unfortunately, this study by Ambrose et al. stayed the only one of its kind investigating in the saturation magnetisation of ultrathin FeCo films. By proper inclusion of error bars the authors pointed out the ambiguity about the saturation magnetisation actually crossing the Slater-Pauling limit, but given numerous studies on increased $\mathrm{Fe}$ moments in quasi-2D systems (see Section II B 1), similar theoretical studies $^{201,202}$ and experimental reports ${ }^{203,204}$ on a moment increase for quasi-2D Co layers, and the high Curie point of FeCo alloys (see Section II A), the increase in the saturation magnetisation of ultrathin $\mathrm{FeCo}$ alloys at room temperature reported by Ambrose and coworkers seems possible. The high sensitivity of the magnetic moment of TM MLs not only to the seed material but also to its texture should be taken into account when attempting to reproduce the results (see, e.g., Moulas et al. reporting on $\mathrm{Fe}_{X} \mathrm{Co}_{1-X}$ MLs on Pt ( $\left.\begin{array}{lll}1 & 1 & 1\end{array}\right)$ substrates $\left.^{205}\right)$.
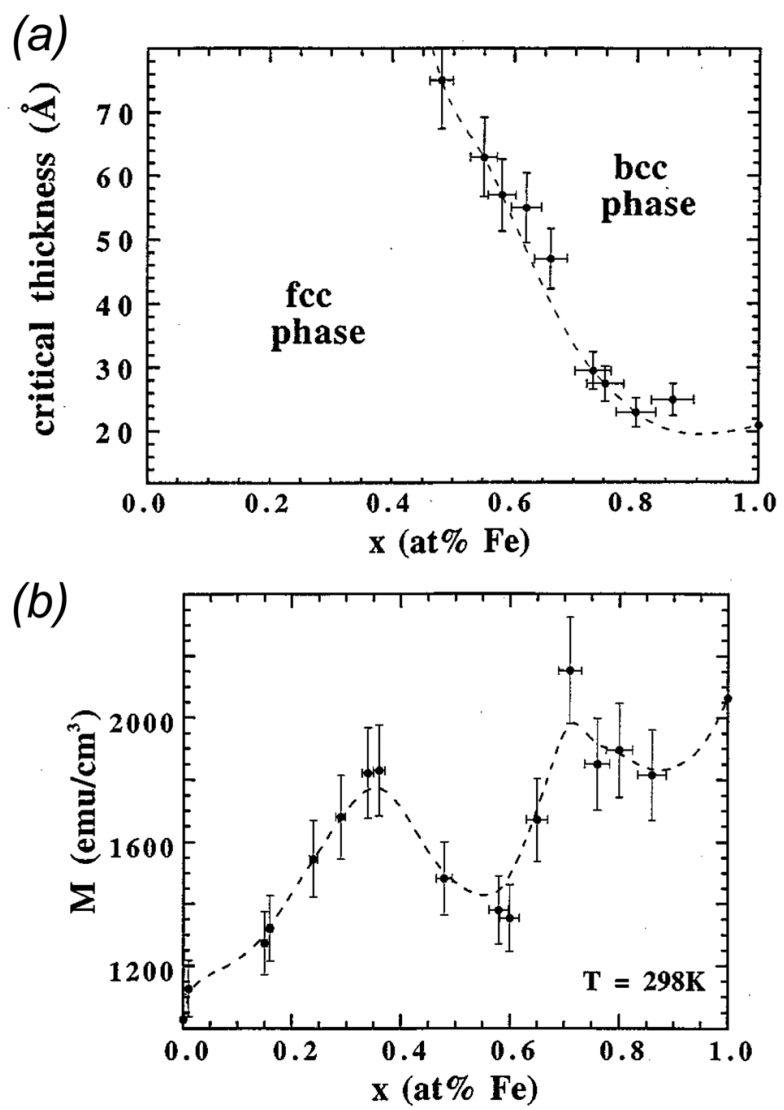

FIG. 15. (a) Critical thickness for the transition from FCC to BCC structure of FeCo alloys MBE-grown by Ambrose and coworkers. (b) Saturation magnetisation values recorded at room temperature for ultrathin $\mathrm{FCC} \mathrm{Fe} \mathrm{X}_{\mathrm{X}} \mathrm{Co}_{1-X}$ layers, revealing a maximum of $\mu_{0} M_{\mathrm{S}}=(2.70 \pm 0.38) \mathrm{T}$ for $X=0.7$. Graphs (a) and (b) are reprinted with permission from J. Appl. Phys. 85, 5066 (1999). Copyright 1999 AIP Publishing LLC. ${ }^{169}$

\section{Summary: TM/NM interfaces, superlattices, and TM-NM alloys}

Summarising the experimental data shows that TM-NM alloys clearly do not yield an improvement of the saturation magnetisation compared to pure Fe or FeCo alloys. For ultrathin high-moment films, one finds a critical thickness limit of about $t_{\mathrm{c}}=2.5 \mathrm{~nm}$, which corresponds roughly to the second peak for parallel coupling in the Ruderman-Kittel-KasuyaYoshida (RKKY) model for indirect exchange coupling in thin films. ${ }^{35}$ The observed high saturation magnetisation in films of $t<t_{\mathrm{c}}$ is believed to come from a narrowing of the $3 d$ band in Fe atoms, exhibiting $m=2.7 \mu_{\mathrm{B}} /$ atom on average, which corresponds to a saturation magnetisation of $\mu_{0} M_{S}^{B C C}=2.7 \mathrm{~T}$ and $\mu_{0} M_{S}^{F C C}=2.6 \mathrm{~T}$ for BCC and FCC ordered Fe, respectively. Thermal instability in thinnest layers causes the Curie temperature to drop dangerously close to room temperature, except for FeCo alloys.

A property of ultrathin TM layers, which was so far ignored, would also favour FeCo-based films over pure Fe: magnetic anisotropy. For ultrathin films, surface anisotropy plays an important role and a strong out-of-plane anisotropy was reported, e.g., for $\mathrm{Fe} / \mathrm{Au}$ nanolaminates ${ }^{176}$ and for $\mathrm{Fe}$ MLs at an Au (l 111 ) interface, ${ }^{206}$ undesired for write pole applications, whereas ultrathin films of FeCo alloys seem not to suffer from these anisotropy issues. ${ }^{169}$

\section{III-V-SC/TM interfaces}

Although no magnetic moment improvement of TMs at III-V SC interfaces has been reported, the moment conservation of $\mathrm{Fe}$ due to the absence of magnetically dead layers is a good incentive to take a second look, particularly since only SCs can be used as substrates - unlike the metallic films discussed above which can only work as seed layers. III-V SCs consist of elements of the third and fifth column of the periodic table:

- the essential group (III) elements are $\mathrm{Al}, \mathrm{Ga}$, In and

- the essential group (V) elements are N, P, As, Sb.

The most widely studied interface is $\mathrm{Fe} / \mathrm{GaAs}$, closely followed by $\mathrm{Fe} / \mathrm{InAs} .{ }^{207,208}$ In both cases, a nearly completely conserved magnetic moment was reported based on the XMCD analyses; however, concerns were raised regarding the origin of the magnetic moment-being the result of an increased orbital moment at the expense of the spin moment (in applied terms: the anisotropy increases and makes the ultra-thin film harder). ${ }^{209}$ Unfortunately, the magnetic moment seems not be conserved for $\mathrm{Co} / \mathrm{GaAs}^{210}$ or $\mathrm{Ni} / \mathrm{GaAs},{ }^{211}$ whereas the latter shows the most severe changes in magnetic anisotropy. ${ }^{212,213}$ Interdiffusion has been identified to be a main concern and was shown to contribute to the formation of undesired $\mathrm{Fe}-\mathrm{Ga}$ phases. ${ }^{214}$

From a pragmatic point of view, given the commercial availability of high-grade wafers, GaAs seems to be the best choice for a high-moment substrate when dealing with Fe and its compounds. Its crystal lattice can conveniently be adjusted by alloying (for instance with $\mathrm{In}^{215}$ ) to further improve the magnetic film structure by tailoring epitaxial strain. But care has to be taken to suppress interdiffusion, 
which impacts on the long-term stability of the high magnetisation.

\section{MnFe}

A purely theoretical study, aiming for the potential utilisation of the high Mn moment, was conducted by Patwari and Victora. ${ }^{216}$ They calculated magnetic moments for alloys of $\mathrm{Mn}_{\mathrm{X}} \mathrm{Fe}_{4-\mathrm{X}} \mathrm{Z}(\mathrm{Z}=\mathrm{N}, \mathrm{C}, \mathrm{B}$, and $\mathrm{Be})$ and found an increase in the Mn moment with expanding lattice parameters, for FCC and BCC structures alike, with a maximum magnetisation polarisation of $\mu_{0} M=2.5 \mathrm{~T}$ and $\mu_{0} M=2.8 \mathrm{~T}$, respectively, as shown in Fig. 16.

So far, no experimental evidence was found for a purely FM coupling in the proposed Mn alloys resulting in an overall moment gain and experimental studies indicate the opposite. $^{217,218}$ Recent studies by Hudl et al. ${ }^{219}$ and Kiss et al. ${ }^{220}$ on crystallised FeMnBSi and amorphous FeMnB alloys, respectively, report inhomogeneous ferromagnetism for certain compositions, however only a small overall saturation magnetisation. Modelling of the according compounds showed the disadvantage of large unit cells and that not all of the TM atoms benefitted from enhanced magnetic moments. In a theoretical study in 2006, Galanakis et al. ${ }^{221}$ showed that Heusler alloys in general also follow the Slater-Pauling curve and thus are unlikely to exceed $\mu_{0} M_{\mathrm{S}}=2.45 \mathrm{~T}$.

\section{E. FeN}

There is one iron compound which seems to ignore the limit given by the Slater-Pauling curve: $\alpha^{\prime \prime}$ ordered $\mathrm{Fe}_{16} \mathrm{~N}_{2}$, which corresponds to a BCT phase with a well ordered $\mathrm{IE}^{222}$ and lattice parameters of $a=5.72 \AA$ and $c=6.29 \AA^{223}$ An initial study by Kim and Takahashi ${ }^{224}$ on films deposited via reactive atomic beam epitaxy in a $\mathrm{N}_{2}$ atmosphere (akin to MBE) reported saturation magnetisations of $\mu_{0} M=2.64 \mathrm{~T}$ for the best achieved Fe-FeN compound [see Fig. 17(a)] and a saturation of $\mu_{0} M \approx 2.8 \mathrm{~T}$ for its $\alpha^{\prime \prime} \mathrm{Fe}_{16} \mathrm{~N}_{2}$ phase was

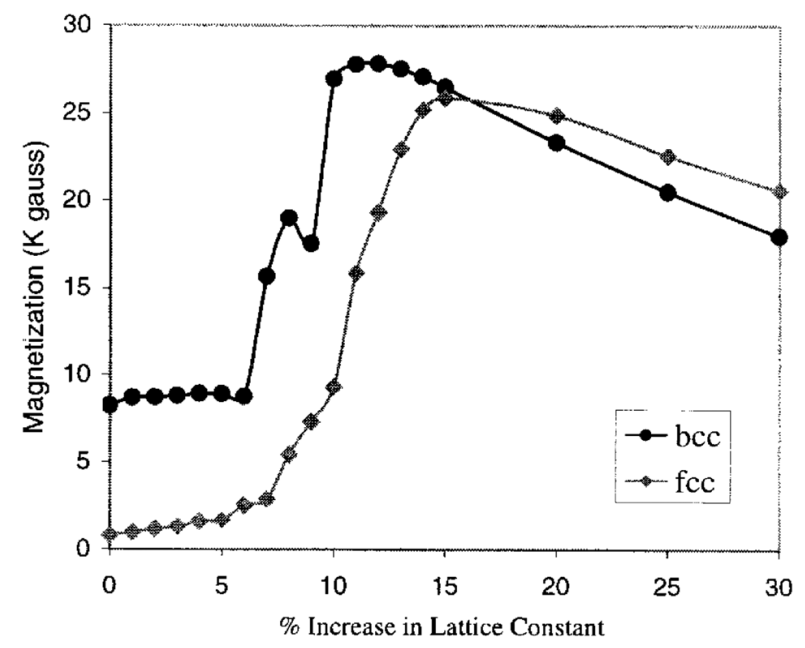

FIG. 16. Calculated magnetisation values of MnFe alloys assumed to order BCC or FCC as reported by Patwari and Victora. Graphs (a) and (b) are reprinted with permission from Patawari and Victora, Phys. Rev. B 64, 214417 (2001). Copyright 2001 the American Physical Society. ${ }^{216}$
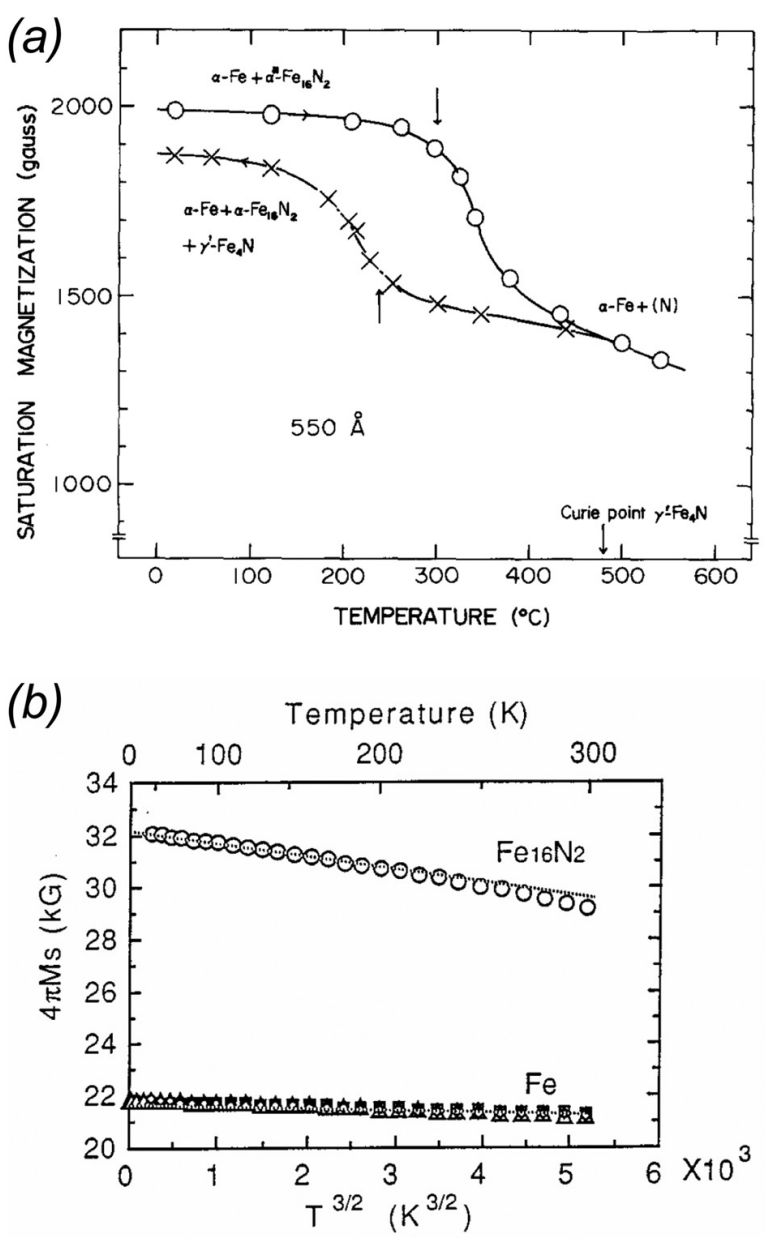

FIG. 17. (a) Extrapolated saturation magnetisation values for different phases of $\mathrm{FeN}$ in an MBE-grown epitaxial film as reported by Kim and Takahashi. (b) Saturation magnetisation values of MBE-grown single-crystal $\alpha^{\prime \prime} \mathrm{Fe}_{16} \mathrm{~N}_{2}$ as reported by Sugita and coworkers. Graph (a) is reprinted with permission from Appl. Phys. Lett. 20, 492 (1972). Copyright 1972 AIP Publishing LLC. ${ }^{224}$ Graph (b) is reprinted with permission from J. Appl. Phys. 79, 5576 (1996). Copyright 1996 AIP Publishing LLC. ${ }^{215}$

extrapolated, corresponding to an average $\mathrm{Fe}$ moment of $\approx 3 \mu_{\mathrm{B}}$ /atom.

Subsequent studies by members of the same research group (i.e., Takahashi, Sugita, Komuro et al.), which continued to rely on the MBE sample synthesis, reported similarly increased saturation values for $\alpha^{\prime \prime} \mathrm{Fe}_{16} \mathrm{~N}_{2}$ and by using $\left(\mathrm{Ga}_{0.8} \mathrm{In}_{0.2}\right)$ As substrates [plus Fe seed layers], an epitaxial single-crystal phase in the $\left(\begin{array}{lll}0 & 0 & 1\end{array}\right)$ orientation [and the $\left(\begin{array}{lll}1 & 1 & 0\end{array}\right)$ orientation, respectively] was stabilised. A magnetic moment of up to $m=3.5 \mu_{\mathrm{B}} / \mathrm{Fe}$ atom was reported for the pure phase-the equivalent of $\mu_{0} M_{\mathrm{S}}=2.9 \mathrm{~T}$ at room temperature and $\mu_{0} M_{\mathrm{S}}=3.2 \mathrm{~T}$ at $5 \mathrm{~K}$ as shown in Fig. $17(\mathrm{~b}){ }^{79,215,225,226}$ In addition to initial VSM measurements the results were corroborated by $\mathrm{FMR}^{227}$ and Moessbauer studies. ${ }^{228}$ There is a still ongoing debate whether or not these values were correct or, at least, why they are not reliably reproducible by other means. ${ }^{223,229-233}$

The Curie point of the metastable $\alpha^{\prime \prime} \mathrm{Fe}_{16} \mathrm{~N}_{2}$ phase is well above room temperature; however, its actual measurement is hampered by its decomposition at $T_{\mathrm{DC}}>470 \mathrm{~K}$ 
according to Takahashi and Shoji. ${ }^{233}$ Sugita et al. ${ }^{79}$ reported this decomposition to happen at $T_{\mathrm{DC}}=670 \mathrm{~K}$ and extrapolated the Curie point to be about $T_{\mathrm{C}}=810 \mathrm{~K}$ (for MBEgrown films). Zhang and co-workers reported the decomposition of the $\alpha^{\prime \prime}$ phase in sputtered films to be as low as $T_{\mathrm{DC}} \approx 500 \mathrm{~K}^{234}$

Studies resorting to alternative fabrication methods nearly exclusively report saturation values below or barely touching the Slater-Pauling limit-in agreement with full potential calculations ${ }^{235-237}$ and spin-polarised linearised muffin-tin orbital calculations, ${ }^{32,238,239}$ in which some of the $\mathrm{Fe}$ atoms in the crystal do show increased magnetic moment, but the average moment does certainly not allow a "giant" magnetisation of the compound:

- synthesis by reactive DC and RF magnetron sputtering, ${ }^{75,240-259}$

- ion-beam sputtering, ${ }^{260-262}$

- ion implantation, ${ }^{263-265}$

- pulsed-laser deposition, ${ }^{266,267}$

- annealing of nitrogen-martensite ${ }^{32,268}$ (the original method for fabrication of $\alpha^{\prime \prime} \mathrm{Fe}_{16} \mathrm{~N}_{2}$ invented by Jack ${ }^{269}$ ),

- halide phase vapour deposition (CVD), ${ }^{270}$

- compacted nanoparticle ensembles ${ }^{271-276}$ (a recent reinvestigation of the FeN system in an effort to harness the large anisotropy for RE-free permanent magnets ${ }^{277}$ ).

There is a number of studies on sputter-deposited FeN thin films, which report saturation magnetisations beyond Slater-Pauling for the $\alpha^{\prime \prime} \mathrm{Fe}_{16} \mathrm{~N}_{2}$ phase in a mix of different FeN phases, ${ }^{278-286}$ but often with large uncertainties and always lower than for the MBE-grown pure $\alpha^{\prime \prime}$ phase. The fact that different groups-applying what seems to be a near-identical sputtering fabrication routine-reported such different results of the magnetic properties, in the range of $2.1 \mathrm{~T}<\mu_{0} M_{\mathrm{S}}<2.8 \mathrm{~T}$, raised considerable doubts regarding data reliability within the magnetism community. Note that the literature base is actually much larger (particularly for sputter-deposited thin films), but a selection was made to include the major research groups in the field. In many of the above listed studies, the layer quality was improved by (post-) annealing, sometimes even aided by the application of a small magnetic field small magnetic field during deposition. The average magnetic moment of $\mathrm{Fe}$ in these experiments was mostly higher than the bulk BCC Fe moment, but nowhere near a "giant" moment. As one of the most recent investigations of FeN thin films fabricated by reactive sputtering, Dirba et al. ${ }^{259}$ also reported a moderate moment increase; however, the authors explicitly state that it has nothing to do with the formation of $\alpha^{\prime \prime} \mathrm{Fe}_{16} \mathrm{~N}_{2}$ but simply lattice expansion due to $\mathrm{N}_{2}$ inclusion ${ }^{287}$ as shown in Fig. 18(a) (in agreement with a similar earlier study by Kusumi et $\left.a l .{ }^{243}\right)$. There is a general trend that recent studies on sputtered FeN report more conservative magnetisation values, particularly those conducted by groups which only recently approached the topic. Although more than one route to produce macroscopic quantities of FeN nanoparticles with a high $\alpha^{\prime \prime} \mathrm{Fe}_{16} \mathrm{~N}_{2}$ content was announced (and patented) within the last 10 years, no commercial supplier puts anything but a conservative number on the magnetisation of their samples. (a)
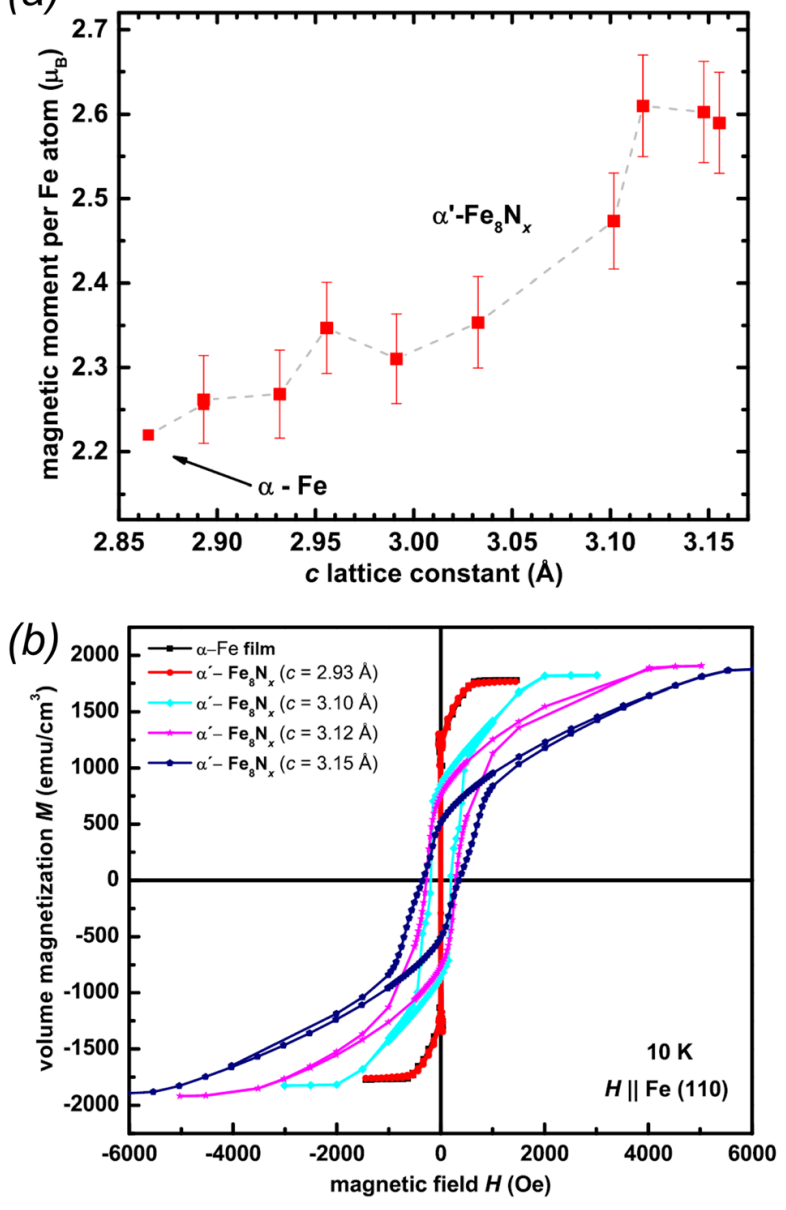

FIG. 18. Results of Dirba et al. on FeN films fabricated by reactive sputtering on $\mathrm{MgO}\left(\begin{array}{lll}1 & 0 & 0\end{array}\right)$ substrates, ordering only in the $\alpha^{\prime} \mathrm{Fe}_{8} \mathrm{~N}$ and $\alpha \mathrm{Fe}$ phases. (a) Magnetic moment per Fe atom with respect to the lattice constant. (b) Increase of saturation magnetisation, required saturation field and hardness with increasing lattice spacing of the Fe atoms. Graphs (a) and (b) are reprinted with permission from J. Appl. Phys. 117, 173911 (2015). Copyright 2015 AIP Publishing LLC. ${ }^{259}$

Critical review articles hesitate to dismiss the idea of the giant magnetic moment, however they never actually support it completely (see, for instance, Refs. 32, 223, 229, and 231). Probably the most comprehensive review on the FeN system, summarising theoretical understanding as well as experimental results up to 1999 , is given by Coey and Smith ${ }^{288}$ and is critical towards the "giant" Fe moment claim. Despite certain progress in getting closer to MBE-quality with plasmasputtering technology [see, for instance, Tian et al. ${ }^{289}$ for growth on $\mathrm{NaCl}\left(\begin{array}{lll}1 & 0 & 0\end{array}\right)$ substrates, Kappaganthu and Sun ${ }^{290}$ for growth on Si ( $\left.\begin{array}{lll}1 & 0 & 0\end{array}\right)$ wafers, and Ji et al. ${ }^{291}$ for growth on Fe-seeded GaAs ( $\left.\begin{array}{lll}0 & 0 & 1\end{array}\right)$ wafers reminiscent of the "golden" MBE recipe by Takahashi and coworkers] no high-moment single-crystal $\alpha^{\prime \prime} \mathrm{Fe}_{16} \mathrm{~N}_{2}$ was stabilised and reliable reproducibility is still an issue. One explanation why the giant moment sometimes occurs and sometimes not is based on the degradation of the compound, i.e., a decomposition of the $\alpha^{\prime \prime} \mathrm{Fe}_{16} \mathrm{~N}_{2}$ into the lower-moment $\alpha^{\prime} \mathrm{Fe}_{8} \mathrm{~N}$ phase. ${ }^{292,293}$ The mixed nature of the FeN compounds, which makes it very hard to actually (quantitatively) determine the $\alpha^{\prime \prime} \mathrm{Fe}_{16} \mathrm{~N}_{2}$ phase for calculating the magnetic moment in 
thicker layers or foils, ${ }^{294}$ and also the limited thickness of MBE-grown samples impacting on the accuracy of saturation magnetisation measurements, still keep the error bars on measured magnetisation values unpleasantly large. First theoretical explanation attempts for a saturation moment beyond the Slater-Pauling limit were given by Ji et al. ${ }^{285,295}$ based on partially localised electron states due to the octahedral structure of FeN clusters in $\alpha^{\prime \prime} \mathrm{Fe}_{16} \mathrm{~N}_{2}$ (see also Sims et $a l .{ }^{296}$ and Shi et $a l .^{297}$ ); however, XMCD measurements which were supposed to corroborate the claim did only confirm that there are increased-moment $\mathrm{Fe}$ sites in ordered $\mathrm{Fe}_{16} \mathrm{~N}_{2}$ and disordered $\mathrm{Fe}_{8} \mathrm{~N}$ crystals alike. ${ }^{298}$ In a 2013 study, Ji et al. ${ }^{299}$ correlated the giant magnetic moment in face-target sputtered $\mathrm{Fe}_{16} \mathrm{~N}_{2}$ thin films to interfacial strain (see also Refs. 300 and 301). An interface-related increase in the magnetic moment of $\mathrm{Fe}$ in the MBE-grown thin films has already previously been mentioned by $\mathrm{Coey}^{222}$ as possible reason for the large saturation magnetisation in those films. After annealing bulk FeN in huge magnetic fields of $\mu_{0} H=9 \mathrm{~T}$ at $T=150^{\circ} \mathrm{C}$ for $20 \mathrm{~h}$, Jiang et al. reported a significant increase of the portion of $\alpha^{\prime \prime} \mathrm{Fe}_{16} \mathrm{~N}_{2}$ in the compound ${ }^{302}$ (at expense of the disordered $\mathrm{Fe}_{8} \mathrm{~N}$ phase); however, no significant magnetisation since the overall $\alpha^{\prime \prime}$ phase content was only $22 \%$ after annealing.

In an effort to stabilise the high-moment phase by alloying, Wang and coworkers ${ }^{303,304}$ investigated the $\mathrm{Fe}_{100-\mathrm{X}-\mathrm{Y}}$ $\mathrm{Ti}_{\mathrm{X}} \mathrm{N}_{\mathrm{Y}}$ system (with $\mathrm{X}=10-12$ and $\mathrm{Y}=5-8$ ), fabricated by reactive co-sputtering, and reported a saturation magnetisation touching the Slater-Pauling limit. Most remarkably, their nanogranular FeTiN films were also very soft $\left(H_{\mathrm{C}}<2 \mathrm{Oe}\right)$ and showed thermal stability at least up to $T \approx 800 \mathrm{~K}$. However, as for pure $\alpha^{\prime \prime} \mathrm{Fe}_{16} \mathrm{~N}_{2}$ films, efforts reproducing the promising results were fruitless. In a subsequent study by Byeon et al. ${ }^{305}$ the compound's softness was confirmed $\left(H_{\mathrm{C}} \approx 3 \mathrm{Oe}\right)$, but neither its magnetisation $\left(\mu_{0} M_{\mathrm{S}} \approx 2 \mathrm{~T}\right.$ ) nor its thermal stability was verified (decomposition of the high-moment phase starting from $T \approx 500 \mathrm{~K}$ ). These results for $\mathrm{Ti}$ are among those of a range of alloying elements like $\mathrm{Al}^{306} \mathrm{Ni},{ }^{307,308} \mathrm{Pt},{ }^{254} \mathrm{Ta}, \mathrm{Zr}$, Hf, and $\mathrm{Nb}$, which do improve softness and thermal stability of the FeN compound, but only yield moderate saturation magnetisation of $\mu_{0} M_{\mathrm{S}} \leq 2.3 \mathrm{~T} .^{288,309}$ There are also high-moment reports for $(\mathrm{Fe}, \mathrm{Co}){ }_{16} \mathrm{~N}_{2}$ by Wang and coworkers; ${ }^{309-311}$ however, lacking definite confirmation by a large base of experiments conducted by different research groups. In contrast to these findings, Sun and Wang, ${ }^{159}$ Liu et al., ${ }^{312}$ and Atiq et al. ${ }^{254}$ reported a lower saturation magnetisation of $\mu_{0} M_{\mathrm{S}} \leq 2.45 \mathrm{~T}$ for the FeCoN system; Takahashi et al. ${ }^{313}$ reported a diminishing thermal stability. However, there is a general agreement that the addition of Co aids the formation of the $\alpha^{\prime \prime} \mathrm{Fe}$ phase. ${ }^{314}$ Partial carbonisation and the creation of a $\mathrm{Fe}_{16} \mathrm{C}_{2}$ phase were under debate for being the origin of a more stabile $\alpha^{\prime \prime} \mathrm{Fe}_{16} \mathrm{~N}_{2}$ phase, ${ }^{315}$ but so far there is no definite proof of the advantages in adding other IEs to the FeN system. ${ }^{316,317}$

Overall, there is still no agreement on the magnetisation of $\alpha^{\prime \prime} \mathrm{Fe}_{16} \mathrm{~N}_{2}$, reported to be $2.1 \mathrm{~T} \leq \mu_{0} M_{\mathrm{S}} \leq 3.2 \mathrm{~T}$, and no unambigious identification of a "giant" Fe moment (e.g., via $\mathrm{XMCD}$ ) unique to it. There is also no known reliable procedure to fabricate thin films of high purity of this metastable phase on an industrial scale. But even with the realisation of this important prerequisite, to eventually replace current FeCo pole tips the following points should definitely be taken into consideration:

- $\alpha^{\prime \prime} \mathrm{Fe}_{16} \mathrm{~N}_{2}$ has repeatedly been reported to be rather hard (with a coercive field strength up to two orders of magnitude larger than bulk permendur ${ }^{318}$ in compacted nanograin ensembles due to a large crystal anisotropy ${ }^{273}$ ), which will be an issue at $\mathrm{GHz}$ write frequencies (only $\mathrm{FeN}$ with low $\mathrm{N}_{2}$ content is confirmed to be a soft ferromagnet concomitant with a lower magnetisation such as bulk BCC $\mathrm{Fe}$ as shown in Fig. 18(b) and, as for the saturation magnetisation of $\alpha^{\prime \prime} \mathrm{Fe}_{16} \mathrm{~N}_{2}$, there is large ambiguity regarding its hardness with a coercive field as low as $H_{\mathrm{C}}=5 \mathrm{Oe}$ assumed for the pure $\alpha^{\prime \prime}$ phase $^{2}$ ).

- The crystal anisotropy of $\alpha^{\prime \prime} \mathrm{Fe}_{16} \mathrm{~N}_{2}$ thin (and particularly ultra-thin) films does not favour the in-plane direction, ${ }^{319,320}$ which would necessitate drastic changes to the write pole fabrication or its general design for optimal HDD operation (in conjunction with the compound being quite hard in the first place, this increases the required external field to $H>0.5 \mathrm{~T}$ for in-plane saturation of an $\alpha^{\prime \prime} \mathrm{Fe}_{16} \mathrm{~N}_{2}$ thin film in the quasi-static case alone).

- The thermal stability of the $\alpha^{\prime \prime} \mathrm{Fe}_{16} \mathrm{~N}_{2}$ phase is nowhere close to industry standards due to the low decomposition temperature, ${ }^{321}$ and particularly in the light of upcoming HAMR drives becomes an unthinkable hazard to device reliability.

In order to verify the general suitability of $\mathrm{Fe}_{16} \mathrm{~N}_{2}$ for use in the HDD write heads, perhaps it would be more fruitful to recall the requirements dictated by what we labelled secondary magnetic properties of pole pieces (see Section ID) rather than a sole focus on saturation magnetisation.

For improved fundamental understanding of this unique material system, it would be highly desirable to repeat the creation of the single $\alpha^{\prime \prime}$ phase grown by MBE (i.e., reactive atomic beam epitaxy), such as done by Takahashi and Sugita et al., to check the influence of different seed materials on the saturation magnetisation. For instance, Yamaguchi et al. found it difficult to grow $\mathrm{Fe}_{X} \mathrm{~N}$ films with $X>3$ via MBE on Si (l $\left.\begin{array}{lll}1 & 1 & 1\end{array}\right)$ substrates $^{322}$ and Ito and coworkers reported only moderate saturation magnetisations of $\mu_{0} M_{\mathrm{S}}<2.4 \mathrm{~T}$ for $\gamma^{\prime}$ $\mathrm{Fe}_{4} \mathrm{~N}$ MBE-grown on $\mathrm{SrTiO}_{3}\left(\begin{array}{lll}0 & 0 & 1\end{array}\right)$ substrates. ${ }^{323}$ Further studies resorting to MBE synthesis should aim for quantifying the influence of epitaxial strain and, provided the fabrication of an unstrained $\alpha^{\prime \prime}$ phase succeeds, if it is $\mathrm{Fe}_{16} \mathrm{~N}_{2}$ which does have a giant magnetisation or not.

\section{F. Heavy RE metals: Pure elements, superlattices, and alloys}

RE metals owe their huge magnetic moment unpaired electrons on the $4 f$ shell and collective FM coupling is not mediated directly, but via the conducting electrons, resulting in the oscillating behaviour of the RKKY coupling. ${ }^{324}$ Many of the RE metals have a magnetic moment per atom far above FM TMs such as FeCo. Nevertheless, there is no 
reason for boundless optimism, since all heavy RE metals with large atomic moment also have large atomic radii, i.e., interatomic spacing, and therefore often only similar saturation magnetisation compared to TMs. They do not order densely packed in the BCC phase, such as many TMs, but hexagonally (quasi) HCP. Their largest disadvantage is their limited operating temperature - they are only FM in the cryogenic temperature regime. In addition, they are highly reactive and prone to degradation. ${ }^{325}$ The RE metals having the highest atomic moment are Dy and Ho. Tb has a slightly reduced magnetic moment, but benefits from a much higher Curie point, and Gd has the largest Curie temperature of all FM lanthanides. Those four RE metals were chosen as representatives of their kind in this review.

\section{Pure heavy RE metals: Ho, Dy, Tb, and Gd}

Valid for bulk RE single crystals of Ho, Dy, Tb, and Gd, the following saturation magnetisations at $T \rightarrow 0 \mathrm{~K}$ were calculated according to magnetic moment values by Rhyne and McGuire $^{326}$ and structural information by Jensen and Macintosh; ${ }^{324}$ points of magnetic transition $\left(T_{\mathrm{C}}, T_{\mathrm{N}}\right.$, and the metamagnetic transition between $\mathrm{AFM}$ and $\mathrm{FM} T_{\mathrm{M}}$ ) are taken from Belov et al.: ${ }^{327}$

- Ho: $\mu_{0} M_{\mathrm{S}}=3.87 \mathrm{~T}, T_{\mathrm{N}}=133 \mathrm{~K}$, and $T_{\mathrm{M}}=20 \mathrm{~K}$.

- Dy: $\mu_{0} M_{\mathrm{S}}=3.82 \mathrm{~T}, T_{\mathrm{N}}=179 \mathrm{~K}$, and $T_{\mathrm{M}}=85 \mathrm{~K}$.

- Tb: $\mu_{0} M_{\mathrm{S}}=3.40 \mathrm{~T}, T_{\mathrm{N}}=230 \mathrm{~K}$, and $T_{\mathrm{M}}=219 \mathrm{~K}$.

- Gd: $\mu_{0} M_{\mathrm{S}}=2.66 \mathrm{~T}$ and $T_{\mathrm{C}}=294 \mathrm{~K}$.

So even taking into account a smaller atomic density, due to the large atomic radii of these $4 f$-based magnetic metals (compared to BCC- or FCC-ordered TMs), they exceed the saturation magnetisation of FeCo-based alloys at cryogenic temperatures. For cryogenic applications they clearly hold the lead, such as for pole pieces made from $\mathrm{Ho}^{328}$ or Dy. ${ }^{329}$ Fig. 19 shows a medley of saturation magnetisation vs. temperature measurements in bulk single crystals to give

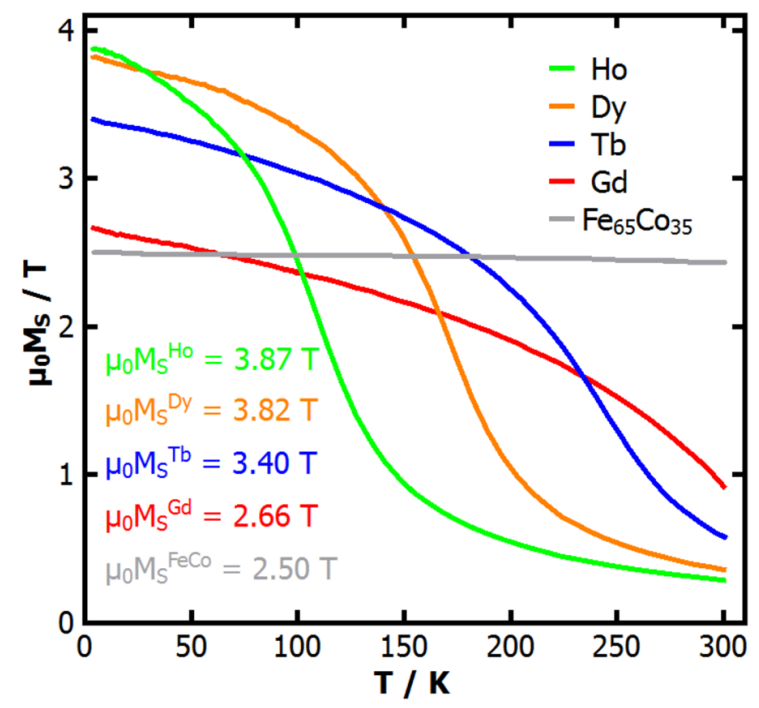

FIG. 19. Saturation magnetisation vs. temperature plots for bulk specimen of $\mathrm{Fe}_{65} \mathrm{Co}_{35}, \mathrm{Gd}, \mathrm{Tb}$, Dy, and Ho. Graph is a modified version of Figure 1 of Ref. 165. Reproduced with permission from Scheunert et al., J. Phys. D: Appl. Phys. 47, 415005 (2014). Copyright (2014) IOP Publishing. an indication on the moment drop with increasing temperature and how the points of magnetic transition shift with increasing field strength (including an FeCo reference).

\section{Inter-RE alloys and laminates}

Due to their chemical similarity heavy RE metals alloy well and for some alloys (such as Gd-rich compositions of DyGd $^{330-333}$ ) parallel coupling of all RE moments and a resulting high saturation magnetisation was observed. ${ }^{334,335}$ However with this more or less simple dilution of magnetic moments, no saturation magnetisation substantially higher than pure Dy can be achieved, ${ }^{165}$ as elucidated at the examples of magnetron-sputtered DyGd and DyTb thin films in Figs. 20(a) and 20(b), respectively. The points of magnetic transition did not improve over the high- $T_{\mathrm{C}}$ element in the alloy. A comprehensive overview on binary inter-heavy-RE alloys was given by Bozorth, ${ }^{336}$ giving information on the magnetic order, Curie (or Néel) points and magnetic moments. One report points out the slight increase of $M_{\mathrm{S}}$ for bulk Ho-Sm alloys with an atomic Sm content of less than

\section{(a)}

\section{DyGd}

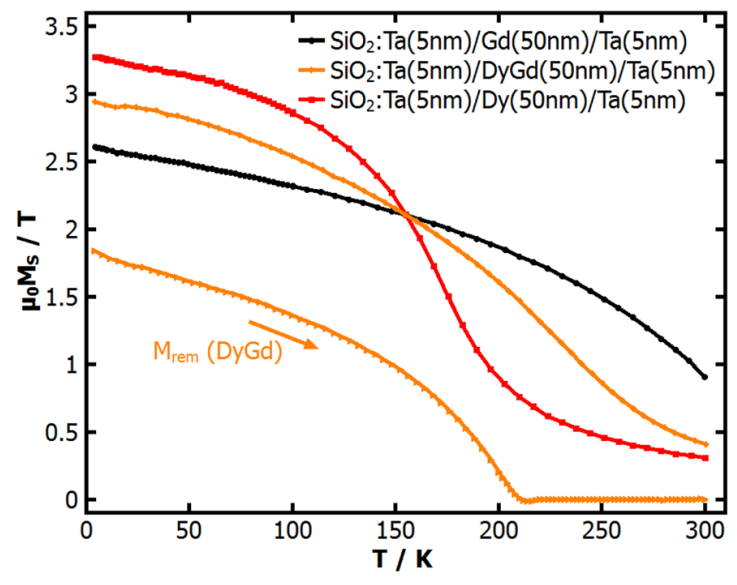

(b)

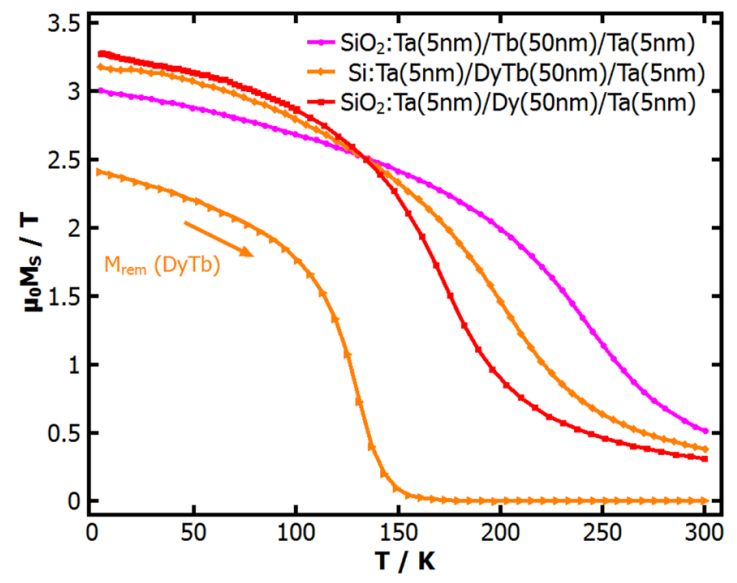

FIG. 20. In-plane high-field magnetisation vs. temperature profiles (and remnant fields) of elemental RE thin films in comparison to inter-RE alloys of (a) DyGd and (b) DyTb. Graph reproduced with permission from Scheunert et al., J. Phys. D: Appl. Phys. 47, 415005 (2014). Copyright (2014) IOP Publishing. ${ }^{165}$ 


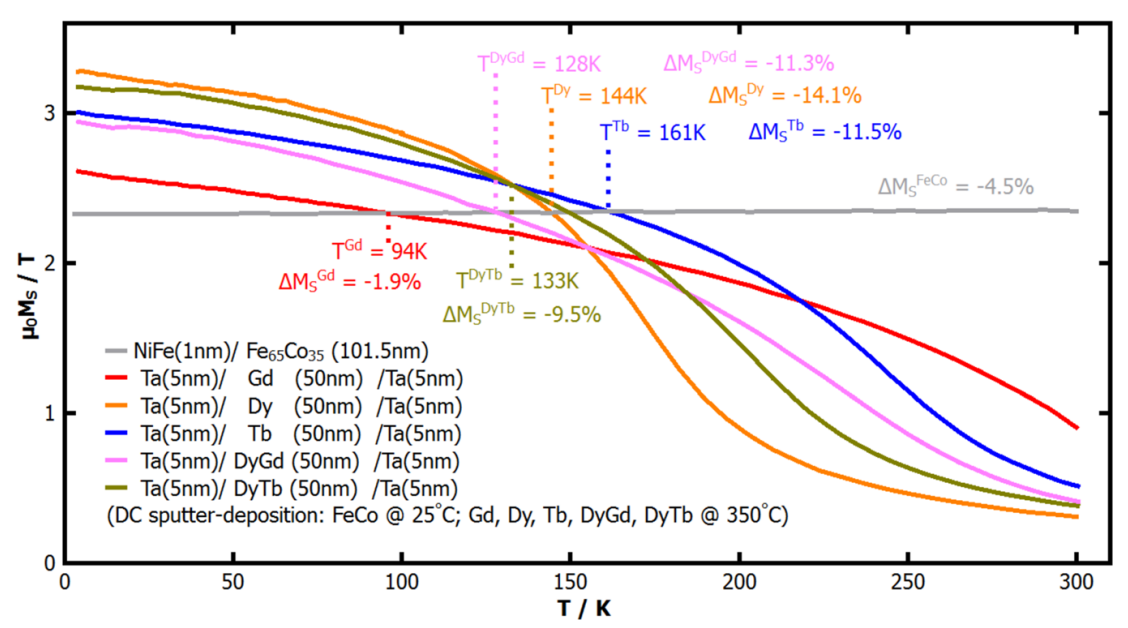

FIG. 21. High-field magnetisation vs. temperature plots for thin films of $\mathrm{Fe}_{65} \mathrm{Co}_{35}, \mathrm{Gd}, \mathrm{Tb}, \mathrm{Dy}, \mathrm{DyGd}$, and DyTb. The marked temperatures indicate where the magnetisations of the RE films match the FeCo reference. Moment losses compared to singlecrystal RE metals are given in relative loss of saturation magnetisation $\Delta M_{\mathrm{S}}$. Graph reproduced with permission from Scheunert et al., J. Phys. D: Appl. Phys. 47, 415005 (2014). Copyright 2014 IOP Publishing. ${ }^{165}$

6\% (Ref. 337) over elemental polycyrstalline Ho, but probably only because the Sm helps aligning the magnetic moment of neighbouring grains. A series of patents on inter-RE alloys of $\mathrm{Dy}-\mathrm{Er}^{338}$ and Ho-Er ${ }^{339}$ suggests tailoring magnetic properties such as softness, yet again no moment gain was achieved $^{340}$ and the magnetic order in Ho-Er alloys, proposed to be FM, was shown to be rather complex by Cowley et al. ${ }^{341}$

A wide variety of RE-RE superlattices has been studied for their magnetic transition and coupling behaviour. ${ }^{342,343}$ Although mostly not in the focus of those studies, their saturation magnetisation measurements show no improvement of the laminate over pure bulk RE saturation magnetisation. If not coupled antiparallel, due to RKKY-described changes in the exchange constant $\mathcal{J}_{\text {RKKY }}$ with layer thickness, resulting moments still do not exceed those of pure elements. Measurements on Dy/Er multilayer stacks might be interpreted to show an increased Dy moment; ${ }^{344}$ however, the experimentalists, Dumesnil et al., pointed out the missing correction for the additional Er moments which brings the overall stack saturation well below pure bulk Dy. Strained Dy thin films in Lu/Dy/Lu trilayer stacks ${ }^{345}$ and Zr/Dy multilayers $^{346}$ were shown to have significantly increased $T_{\mathrm{C}} \approx 170 \mathrm{~K}$ and a completely suppressed AFM phase, however it was later shown for strained Dy in Ta/Dy/Ta trilayers, ${ }^{347}$ which exhibits similar behaviour that the $T_{\mathrm{C}}$ increase comes at the expense of magnetic moment. A similar observation was made for $\mathrm{Tb}$ in $\mathrm{Ta} / \mathrm{Tb} / \mathrm{Ta}$ trilayers with a direct transition to the FM state at $T_{\mathrm{C}}=216 \mathrm{~K}$ concomitant with a moment drop. ${ }^{165}$

The saturation magnetisation (or at least high-field magnetisation) of the RE thin films depends very sensitively on the crystal structure. For most seed materials or substrates, the RE metals are strained and tend to order FCC (at least in proximity to the seed interface $)^{348,349}$ and it was found that the strained HCP phase and in particular the FCC phase lead to poor magnetic moments and strongly reduced saturation, shown for $\mathrm{Gd},{ }^{350-352} \mathrm{Dy},{ }^{347}$ and $\mathrm{Tb} .{ }^{165}$ Only the growth of the unstrained HCP equilibrium phase promotes high magnetic moments, which normally requires annealing of the specimen $^{353}$ and/or perfect-match seed layers. ${ }^{342,354}$

The medley of (in-plane) magnetisation vs. temperature profiles of selected RE thin films in Fig. 21 allows for a quick comparison to bulk specimen (as in Fig. 19): there is a moment loss for most RE thin films, even more severe than for FeCo layers of similar thickness $(t \approx 50-100 \mathrm{~nm})$.

\section{Gd/W laminates}

There are a large number of publications on improved magnetic properties of REs in laminates with non-FM metals. With an emphasis on large saturation moments, there are two studies on Gd/W laminates by Heys, Donovan and coworkers $^{355,356}$ which stand out (see Fig. 22). They reported a massive increase in the Gd moment of up to $m=34 \mu_{B}$ /atom. Although this is a gigantic increase in the magnetic moment, which is far from any theoretical prediction, the overall saturation magnetisation of the entire laminate was still below single crystal bulk $\mathrm{Gd}$ and can be extrapolated to $\mu_{0} M_{\mathrm{S}}=2.57 \mathrm{~T}$, measured at $T=5 \mathrm{~K}$. No information was given on the Curie temperature, which is likely lower than single-crystal Gd and thus well below room temperature. However, there is a number of studies reporting enhanced Curie points for strained Gd films grown on Mo, ${ }^{357}$ which could also apply in this case. Contrary to this speculation are recent findings by Bertelli et al. ${ }^{352}$ who correlated an extraordinary increase of the Curie temperature in Ta-sandwiched sub-10 nm FCC Gd films with epitaxial strain, since this phenomenon is accompanied by a severe moment drop, rendering the increased working temperature effectively useless for high-moment applications. ${ }^{165}$

Unfortunately, no subsequent study succeeded in reproducing the initial giant magnetic moment results ${ }^{358,359}$ which raises considerable doubts on the high-moment claim, and magnetisation vs. temperature measurements of those studies indicate Curie temperatures similar or lower than bulk Gd. There is no other study on RE-non-magnetic-metal laminates which would suggest sufficient moment enhancement yet an improved saturation magnetisation.

\section{Gd-implanted GaN}

There is no report on a general moment conservation at III-V SC interfaces for RE metals, such as for Fe (see Section IIC). However, there are encouraging individual reports on Gd-implanted GaN. Truly "colossal" Gd moments of up to $m \approx 4000 \mu_{B}$ /atom have been reported by Dhar et al. $^{360}$ for Gd-implanted reactive MBE-grown GaN 
(a)

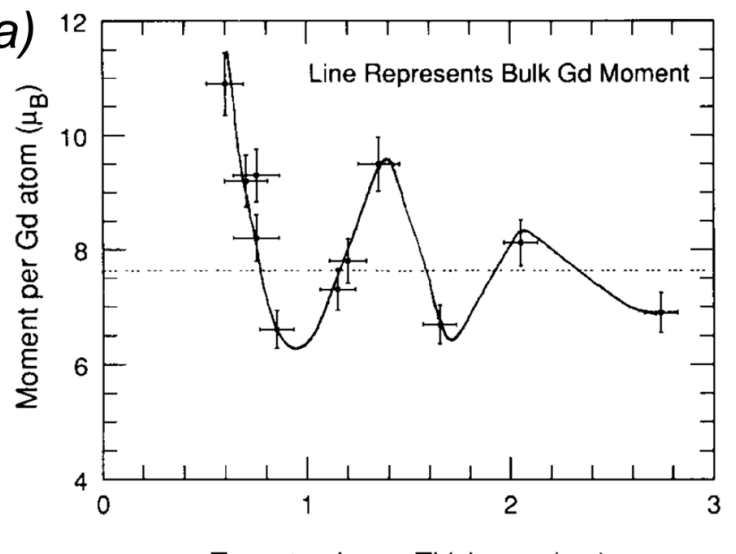

Tungsten Layer Thickness ( $\mathrm{nm}$ )

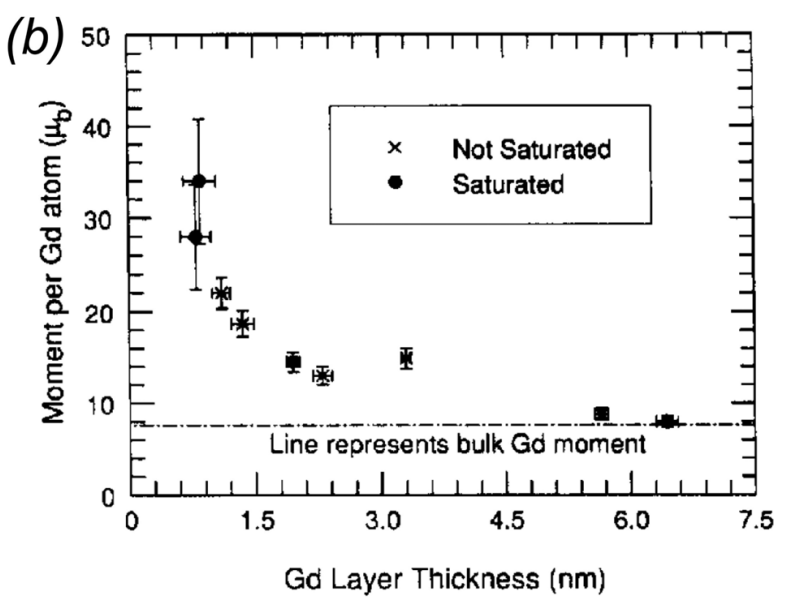

FIG. 22. Magnetic moments of $\mathrm{Gd}$ atoms in $\mathrm{Gd} / \mathrm{W}$ laminates as reported by (a) Heys and Donovan $\left(t_{\mathrm{Gd}}=4.4 \mathrm{~nm}\right)$ and (b) Heys et al. $\left(t_{\mathrm{W}}=1.7 \mathrm{~nm}\right)$. The magnetic moments were extrapolated from VSM measurements conducted at $T=5 \mathrm{~K}$. Graph (a) is reprinted from Heys and Donovan, J. Magn. Magn. Mater. 126, 326 (1993). Copyright 1993 Elsevier. ${ }^{355}$ Graph (b) is reprinted from Heys et al., J. Magn. Magn. Mater. 131, 265 (1994). Copyright 1994 Elsevier. ${ }^{356}$

layers on sapphire substrates. Those magnetic moment values were extrapolated from SQUID magnetisation measurements of the entire specimen at $T=2 \mathrm{~K}$ and $T=300 \mathrm{~K}$ as shown in Figs. 23(a) and 23(b), respectively. Saturation measurements however revealed a very modest magnetisation per volume of only $\mu_{0} M \approx 0.01 \mathrm{~T}$. In a subsequent study by Dhar et al., ${ }^{361} \mathrm{Gd}$ was implanted by focussed ion beam (FIB), with even higher magnetic moments per Gd atom of $m \approx 5000 \mu_{\mathrm{B}} /$ atom at $T=4 \mathrm{~K}$ and $m \approx 3300 \mu_{\mathrm{B}} /$ atom at room temperature. Again, for the whole sample the highest magnetic polarisation measured was quite modest with $\mu_{0} M \approx 0.04 \mathrm{~T}$. This study also reported an FM phase at room temperature which was later confirmed by XMCD measurements. ${ }^{362}$ From magnetisation measurements on metalorganic vapour phase epitaxy (MOVPE)-grown GaN thin films with FIB-implanted Gd, Hejtmanek et al. ${ }^{363}$ extrapolated the Curie temperature to be $T_{\mathrm{C}} \approx 700 \mathrm{~K}$; however, the "colossal" magnetic moment of Gd could not be confirmed.

A conclusive theoretical model for the origin of the "colossal" Gd moment has yet to be found ${ }^{364,365}$ and is complicated by the coexistence of multiple FM phases. ${ }^{366}$ Dhar et al. ${ }^{360}$ suggested long-range polarisation of the GaN matrix
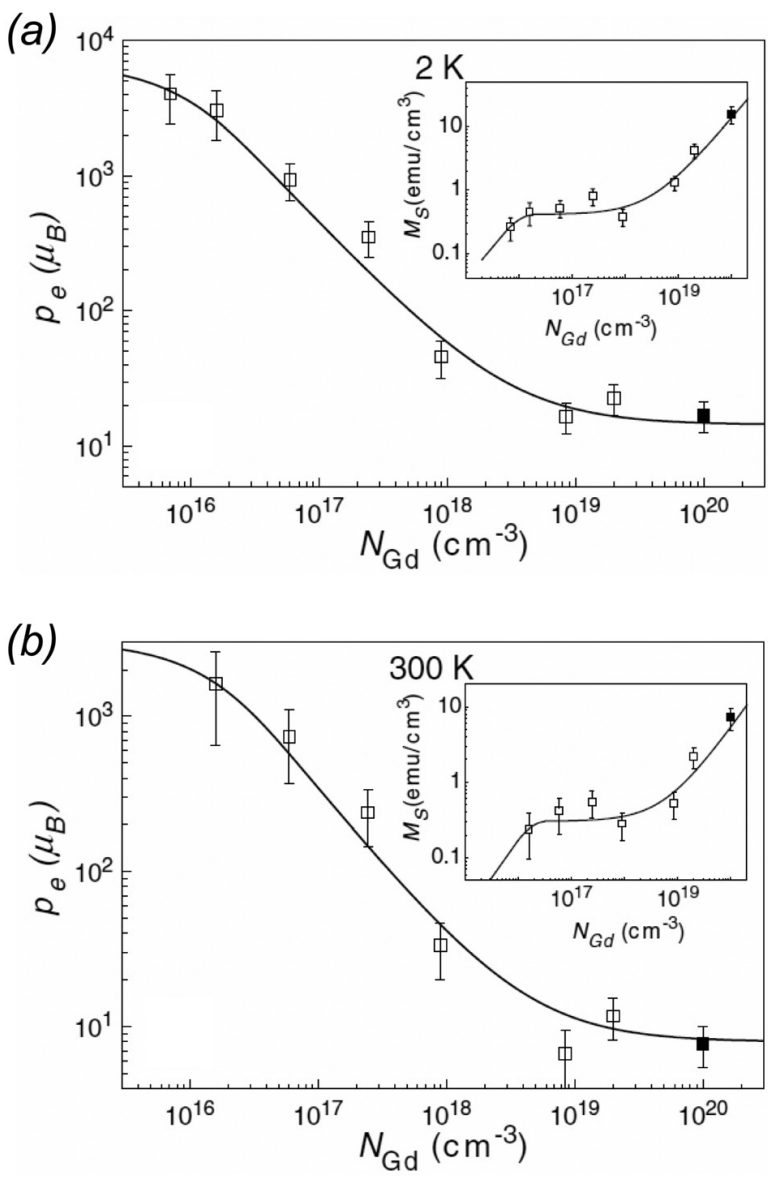

FIG. 23. Extrapolated magnetic moment values of Gd atoms implanted in MBE-grown GaN layers with the original saturation magnetisation measurement in the inset, recorded at (a) $T=2 \mathrm{~K}$ and (b) $T=300 \mathrm{~K}$. Graphs (a) and (b) are reprinted with permission from Dhar et al., Phys. Rev. Lett. 94, 037205 (2005). Copyright 2005 the American Physical Society. ${ }^{360}$

by the atomic moment of the $\mathrm{Gd}$, which is supported by the work done by Khaderbad et al. ${ }^{367}$ on the influence of defect reduction by annealing the Gd-GaN specimen. Further explanations were attempted by Dalpian and Wei, ${ }^{368}$ suggesting an electron-induced stabilisation of an FM phase; Gohda and Oshiyama, ${ }^{369}$ suggesting intrinsic ferromagnetism due to cation vacancies; and Liu et al. ${ }^{370}$ who also suggested defect-induced coupling.

More recent experimental studies on RE-implanted $\mathrm{GaN}^{371,372}$ (and $\mathrm{AlGaN}^{373}$ ) report a variety of complex magnetic structures and confirm FM ordering of some other doping elements (such as Dy). But these results neither undoubtedly confirmed a "colossal" moment nor seemed the reproduction of the FM systems a fully developed method ${ }^{374}$ and impurities as well as the formation of RE oxides were found to be another complication. ${ }^{375}$

In summary, it can be said that, even assuming a "colossal" moment for Gd atoms (and large spontaneous magnetic moments of other heavy RE metals), the overall saturation magnetisation is far too small for high-moment applications. Theoretical understanding so far contradicts potential use in future devices, as the additional magnetic moment requires a large surrounding $\mathrm{GaN}$ matrix. Unfortunately, there is no study on the potential of $G a N$ as 
seed layer material for the growth of high-moment Gd thin films, but a study by Xiao et al. ${ }^{376}$ on the electronic properties of $\mathrm{Gd}$, evaporated on a $\mathrm{GaN}\left(\begin{array}{llll}0 & 0 & 0 & 1\end{array}\right)$ surface, reports interdiffusion and the formation of a metallic Ga-phase-a clear hinderance for smooth high-moment thin films.

\section{Summary: Heavy RE metals, alloys, and compounds}

With their outstandingly high magnetic moment per atom heavy RE metals such as Dy, Ho, Tb, and Gd hold the lead for any bulk high moment application at cryogenic temperatures, with $\mathrm{Dy}^{377}$ and $\mathrm{Ho}^{328}$ mostly being the material of choice at lowest temperatures $T \rightarrow 0 \mathrm{~K}$ and $\mathrm{Gd}$ the favourite material for near room temperature applications due to its high Curie point. ${ }^{93}$ Attempts to increase the magnetic moment of RE atoms in thin films by means of straining the unit cell were unsuccessful, since-unlike TMs-RE metals "can already be considered isolated moments, owing their large atomic radii and spontaneous moments on the wellshielded $4 f$ shell, and changes in the interatomic distance result in magnetic moment changes being subject to the oscillatory nature of the Ruderman-Kittel-Kasuya-Yoshida (RKKY) coupling."165

Alloys of REs with FM TMs ${ }^{378}$ or non-magnetic materials $^{379}$ always lead to a reduction of the saturation magnetisation due to AFM coupling, inhibited coupling or just dilution of the RE moments. Inter-RE alloys were shown to couple FM; however, there is no confirmed report on a substantial moment increase over pure RE metals. There is surprisingly little data available on the magnetic moments of thin and ultrathin layers of REs or laminates for most studies have an emphasis on the magnetic ordering and coupling behaviour. The overall theme is a decrease of saturation magnetisation of thinner RE films, often explained in terms of granularity and strain issues ${ }^{347,350}$ or non-beneficial coupling to adjacent layers. ${ }^{380}$ Laminates, except for isolated reports on $\mathrm{Gd} / \mathrm{W}$, also show decreasing RE moments due to AFM coupling with magnetic $\mathrm{TMs}^{381}$ and for non-magnetic spacers, magnetic coupling predominantly follows RKKY behaviour, i.e., the sign of the exchange constant oscillates with spacer thickness and so does the measured (decreasing) magnetic moment. ${ }^{382}$ The Curie points for nearly every laminate or alloy are below the bulk value, those for thin films of RE are believed to drop as a result of strain or structural changes. Exceptions are severe changes in the magnetic structure for strained Dy and Tb, ${ }^{165,347,383}$ suppressing the AFM phase and raising the $T_{\mathrm{C}}$, unfortunately at the expense of magnetic moment.

\section{G. Combined RE-TM systems}

Thinking about the advantage of heavy RE metals, their large magnetic moment, and the benefit of a large Curie temperature for TMs, there is the natural conclusion to combine the two material classes and harnessing the best of both in a hybrid structure. However, due to the different origin of their ferromagnetism, chemical differences and fabrication issues, merging them is very difficult and, when aiming for high magnetic moments, can be considered rather fruitless so far.

\section{RE-TM/RE-NM alloys and direct laminates}

One way to combine $3 d$ and $4 f$ ferromagnets, and technologically the first one to become available, is to alloy them into inter-RE-TM alloys. A comprehensive review of compounds and alloys of $3 d$ TMs and REs from 1977 by Buschow $^{378}$ and a more recent one from 1997 by Andreenko and Nikitin ${ }^{384}$ with an emphasis on amorphous alloys provide a good overview. In nearly all cases, the TM and the RE couple with their moments in an antiparallel arrangement, resulting in AFM, FIM, or more complicated metamagnetic structures. It can be summarised that, as a result of the antiparallel coupling, the resulting alloy does suffer from a reduced saturation magnetisation which drops particularly quickly with increasing temperature due to compensation issues of the two involved sublattices, reflected in, e.g., the compensation point $T_{\text {comp. }}{ }^{35}$ Only for a large RE content their big magnetic moment generates large saturation magnetisation, yet it is always lower than for the pure RE and the gain in $T_{\mathrm{C}}$ is rather small; if there is any. On the opposite end of the scale, for TM-rich alloys the Curie temperature $T_{\mathrm{C}}$ drops quickly when adding a RE and so does the magnetic moment. What is desired in magneto-optical recording and for permanent magnets, large anisotropies due to the unique interaction of the two different sublattices and strictly antiparallel coupling, seems detrimental to achieving a large saturation magnetisation. Although NMs were found to be readily polarised by adjacent $\mathrm{RE}$ atoms, ${ }^{385}$ this effect is only relevant for very low RE concentration and there is no gain in terms of saturation magnetisation over elemental REs. The same applies to Sc-RE alloys $\mathrm{Sc}_{1-X} \mathrm{Gd}_{\mathrm{X}}$ and $\mathrm{Sc}_{1-\mathrm{X}} \mathrm{Dy}_{\mathrm{X}}$ $(\mathrm{X}<0.01) .{ }^{386}$ Loebich and $\mathrm{Raub}^{387}$ showed that Pd in binary $\mathbf{R E}-\mathbf{N M}(\mathrm{RE}=\mathrm{Gd}$, Dy, and Ho) did not yield similarly beneficial moment enhancements as reported for TM-NM alloys, as shown in Figs. 24(a) and 24(b) for GdPd and DyPd, respectively. Similarly disappointing behaviour was observed for RE-Ru ${ }^{388}$ and RE-Rh alloys. ${ }^{389-392}$

However, among the latter, one ternary alloy system was found in 2014 which showed signs of FM coupling: DyRhNi (more specifically: the (DyRh) ${ }_{85} \mathrm{Ni}_{15}$ composition). ${ }^{393}$ Although its saturation magnetisation is only $\mu_{0} M_{\mathrm{S}}=1.7 \mathrm{~T}$ and its Curie temperature only $T_{\mathrm{C}}=22 \mathrm{~K}$, excluding it from considerations of an $\mathrm{FeCo}$ alternative, it is worth being highlighted for its remarkable softness and for being one of only two reported cases of parallel RE-TM coupling in an alloy (the second one is discussed in Section II G 2). There is yet no in-depth investigation of its magnetic structure and what precisely causes its peculiar behavior.

A second way of combining $3 d$ and $4 f$ ferromagnets has become available due to progress in thin-film deposition, allowing otherwise hard-to-alloy elements in novel compounds, ${ }^{394}$ but also allowing direct RE-TM laminates. The term "direct" refers to both materials sharing one interface, without a non-magnetic spacer or anything else separating them. There are plenty of studies on the magnetic properties of thin film systems of TMs such as Fe, Ni, Co, and RE metals, such as Gd, Dy, Tb, and Ho being stacked in superlattices. ${ }^{381,395-398}$ Although the overall magnetic moment played a minor role in those investigations, there is a clear trend of 
(a)

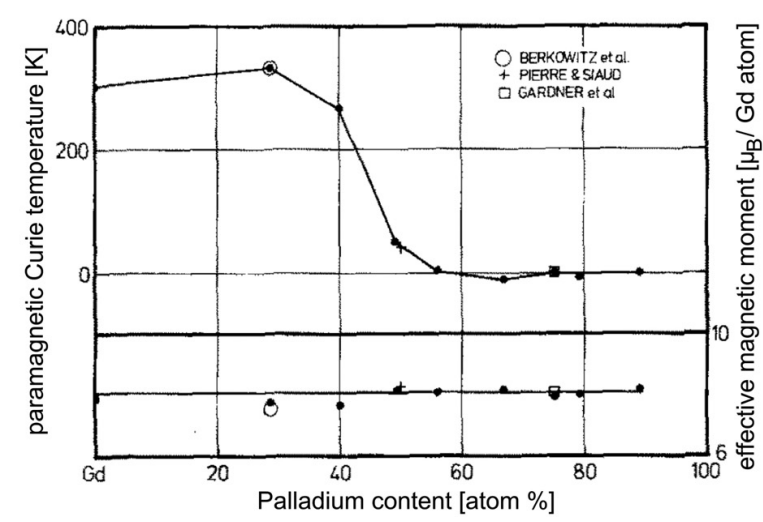

(b)

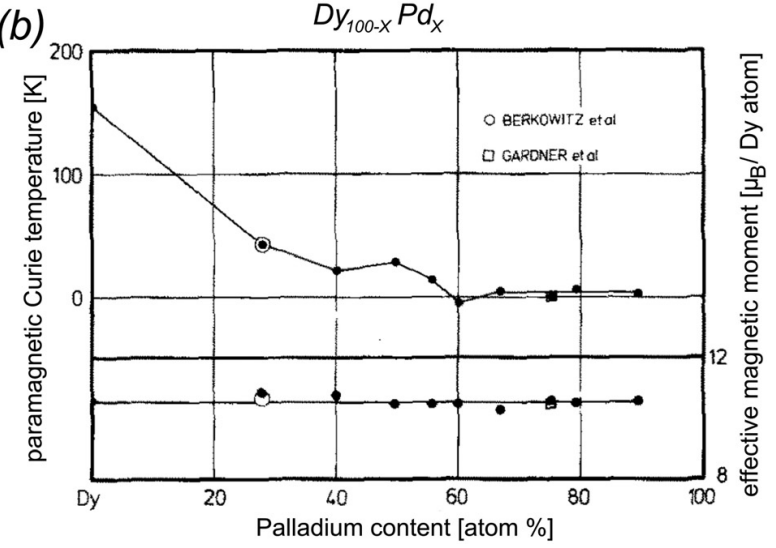

FIG. 24. Curie temperature (left scale) and magnetic moment of the RE (right scale) dependence on the Pd content of (a) GdPd and (b) DyPd alloys. Graphs (a) and (b) are reprinted with translated scales from Loebich, Jr. and Raub, J. Less-Common Met. 31, 111 (1973). Copyright 1973 Elsevier. ${ }^{387}$

all those systems to share antiparallel coupling of the TM to the RE, i.e., a saturation magnetisation drop, and no improvement in the $T_{\mathrm{C}}$. Such as for their alloy counterparts, many of the resulting RE-TM systems were shown to exhibit remarkable anisotropies, which makes them suitable candidates for magneto-optical recording which requires thin films with an out-of-plane easy axis. ${ }^{399,400}$

Summarising the above, there are no confirmed reports on a RE-TM/RE-NM alloy or direct laminate where the saturation magnetisation at room temperature would exceed that of FeCo or that of Dy at cryogenic temperatures. Secondary properties of resulting FIM, AFM, or metamagnetic materials, apart from low overall saturation magnetisation, are low permeability and often high coercivity — which are responsible for the success of strong permanent magnets such as $\mathrm{SmCo}_{5}$ or $\mathrm{FeNdB}$ - but which are unsuitable for write pole applications. A possible way to avoid completely antiparallel coupling in an alloy might be to employ extra "spacers" in a compound (small interstitial atoms which impact on the unit cell size and crystal structure), such as $\mathrm{H}_{2}, \mathrm{~B}, \mathrm{P}, \mathrm{C}, \mathrm{N}_{2}$ or perhaps some of the light REs to achieve a parallel magnetic coupling between two FM sublattices (in analogy to the case of ferrite and austenite steel which, while sharing the same chemical composition, order FM and PM, respectively, due to the formation of $\alpha$ - and $\gamma$-Fe sublattices). However, both, TMs and REs on their own, were shown to react extremely sensitive to changes in their interatomic spacing or electronic structure, which makes the outcome of such ternary compounds rather unpredictable. And for laminates, certain ML-thin spacing layers were proposed to mediate FM coupling. Candidates for both options, IEs in an alloy and a spacing layer in a laminate, will be discussed in this section.

\section{RE-Mn}

A study on arc-melted $\mathrm{RE}_{6} \mathrm{Mn}_{23}$ alloys by DeSavage et al. ${ }^{401}$ reported increased magnetic moments for $\mathrm{Gd}$ in $\mathrm{Gd}_{6} \mathrm{Mn}_{23}$ complex alloys at room temperature. Gd played an outstanding role in the alloy series, for every other RE under investigation clearly coupled antiparallel to the Mn resulting in reduced moments as reported before by Hubbard et al ${ }^{402}$ The observed moment gain of $\mathrm{RE}_{6} \mathrm{Mn}_{23}$ was explained by FM coupling of the exited spin state of Gd to the Mn spin. The moment of each $\mathrm{Gd}$ atom was determined to be $m=8.6 \mu_{B} / \mathrm{Gd}$ atom, see Fig. 25(a), which corresponds to $m=54.7 \mu_{B} / \mathrm{Gd}_{6} \mathrm{Mn}_{23}$ unit cell. ${ }^{403}$ This is equivalent to a rather modest saturation magnetisation of only $\mu_{0} M_{\mathrm{S}}=0.3 \mathrm{~T}$ for the entire alloy.

A subsequent study on the RE-Mn alloys of various compositions by Kirchmayr and Steiner ${ }^{404}$ confirmed a moment increase for $\mathrm{Gd}$ in $\mathrm{Gd}_{6} \mathrm{Mn}_{23}$, which again was explained as an FM coupling of the RE to the Mn. ${ }^{405}$ The Curie point was found to be well above room temperature with $T_{\mathrm{C}}=489 \mathrm{~K}$, see Fig. 25(b). A fundamental assumption in all studies was an unchanged moment for $\mathrm{Mn}$ of $m=0.4 \mu_{\mathrm{B}} / \mathrm{Mn}$ atom, derived from the $\mathrm{Lu}_{6} \mathrm{Mn}_{23}$ alloy, ${ }^{401}$ which lacked further experimental evidence and was contradicted in a recent study by Zhang. ${ }^{406}$

Following up on doubts regarding the FM coupling of $\mathrm{Gd}$ and $\mathrm{Mn}$ in $\mathrm{Gd}_{6} \mathrm{Mn}_{23}$, as outlined and corroborated by polarised-neutrons experiments in a 1979 study by Delapalme et al., ${ }^{407}$ a more recent and particularly comprehensive study by Lemoine ${ }^{403}$ also suggests FIM ordering, which appears much more reasonable giving the normally antiparallel coupling of heavy RE metals and Mn. This study also highlights the much higher magnetic moment per unit cell for $\mathrm{Ho}_{6} \mathrm{Mn}_{23}$, compared to $\mathrm{Gd}_{6} \mathrm{Mn}_{23}$, which is $m=64.4 \mu_{\mathrm{B}} /$ unit cell and comes at the expense of a slightly reduced temperature for the point of magnetic transition, $T_{\mathrm{C}}=445 \mathrm{~K}$, as illustrated in Table II. Owing its outstanding magnetic structure among RE-TM alloys, the GdMn system might still be a good starting point for the creation of a highmoment alloy making additional use of interstitial atoms such as in GdMnP, which will be discussed in Section II G3.

\section{RE/IE/TM and RE/Cr/TM systems}

In 2006, Zoto and Mankey ${ }^{408}$ suggested to couple $\mathrm{Ni}$ and Gd parallel in a trilayer stack via a mediating spacer from Pd. Expecting RKKY coupling, they altered the Pd spacer layer thickness $\left(0.5 \mathrm{~nm}<t_{\mathrm{Pd}}<2.5 \mathrm{~nm}\right)$ to achieve a desirable exchange constant $\mathcal{J}_{\text {RKKY }}$ promoting FM coupling of the $\mathrm{Gd}$ and the Ni. However, they only observed AFM coupling and hence moment reduction for Pd layer thicknesses of $t_{\mathrm{Pd}}<2.5 \mathrm{~nm}$, and no coupling at all for $t_{\mathrm{Pd}}>2.5 \mathrm{~nm}$. 
(a)

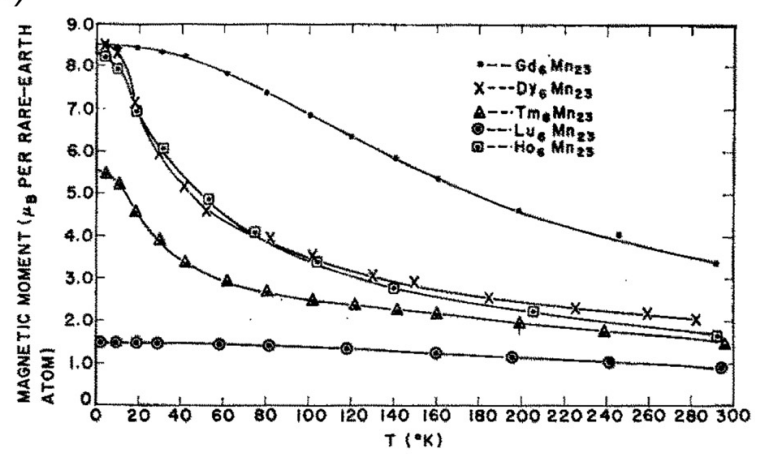

(b)

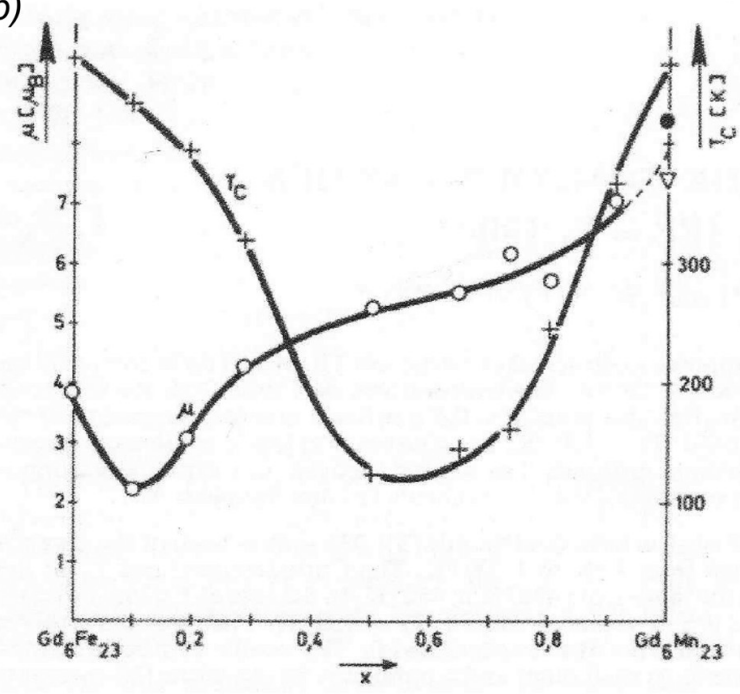

FIG. 25. (a) The magnetic moment per atom values of $\mathrm{Gd}_{6} \mathrm{Mn}_{23}$ as initially reported by DeSavage et al. in 1965. (b) Magnetic moments and Curie temperatures as reported in a subsequent study on $\mathrm{Gd}_{\mathrm{X}} \mathrm{Mn}_{1-\mathrm{X}}$ and $\mathrm{Gd}_{\mathrm{X}} \mathrm{Fe}_{1-\mathrm{X}}$ by Kirchmayr and Steiner in 1971. Note, in both graphs the magnetic moment values are only given for the RE atoms. Graph (a) is reprinted with permission from J. Appl. Phys. 36, 992 (1965). Copyright 1965 AIP Publishing LLC. ${ }^{401}$ Graph (b) is reprinted with permission from Kirchmayr and Steiner, J. Phys. Colloq. 32, 665 (1971). Copyright 1971 EDP Sciences. ${ }^{404}$

But the idea of using an intermediate atom to promote an effective FM exchange coupling between the RE and the TM that can stabilise the RE moment to yield technologically relevant Curie temperatures and capitalise on the large

TABLE II. Overview of structural and magnetic properties of $\mathrm{RE}_{6} \mathrm{Mn}_{23}$ alloys as reported by Lemoine. ${ }^{403}$

\begin{tabular}{lccccc}
\hline \hline & $\begin{array}{c}\text { Unit cell } \\
\text { volume } \\
{\left[\AA^{3}\right]}\end{array}$ & $\begin{array}{c}\text { Density } \\
{\left[\mathrm{g} \mathrm{cm}^{-3}\right]}\end{array}$ & $\begin{array}{c}\text { Trans. } \\
\text { temp. } \\
{[\mathrm{K}]}\end{array}$ & $\begin{array}{c}\text { Saturation } \\
\text { moment } \\
{\left[\mu_{\mathrm{B}} \mathrm{fu}^{-1}\right]}\end{array}$ & $\begin{array}{c}\mu_{0} M_{\mathrm{S}} \\
{[\mathrm{T}]}\end{array}$ \\
\hline $\mathrm{Y}_{6} \mathrm{Mn}_{23}$ & 1931.2 & 6.18 & 505 & 13.3 & 0.08 \\
$\mathrm{Nd}_{6} \mathrm{Mn}_{23}$ & 2039.7 & 6.93 & 441 & 16.1 & 0.09 \\
$\mathrm{Sm}_{6} \mathrm{Mn}_{23}$ & 1997.5 & 7.20 & 456 & 11.3 & 0.07 \\
$\mathrm{Gd}_{6} \mathrm{Mn}_{23}$ & 1968.6 & 7.45 & 489 & 54.7 & 0.32 \\
$\mathrm{~Tb}_{6} \mathrm{Mn}_{23}$ & 1928.9 & 7.63 & 457 & 49.6 & 0.30 \\
$\mathrm{Dy}_{6} \mathrm{Mn}_{23}$ & 1906.2 & 7.80 & 447 & 58.1 & 0.36 \\
$\mathrm{Ho}_{6} \mathrm{Mn}_{23}$ & 1887.8 & 7.93 & 445 & 64.4 & 0.40 \\
$\mathrm{Er}_{6} \mathrm{Mn}_{23}$ & 1867.2 & 8.07 & 430 & 50.7 & 0.32 \\
$\mathrm{Tm}_{6} \mathrm{Mn}_{23}$ & 1844.1 & 8.20 & 441 & 35.6 & 0.23 \\
$\mathrm{Lu}_{6} \mathrm{Mn}_{23}$ & 1813.2 & 8.47 & 398 & 11.2 & 0.07 \\
\hline \hline
\end{tabular}

RE moments was subsequently pursued by several groups. The mediators should in addition to stabilizing the RE moment also ideally have a net moment aligned FM with the $\mathrm{TM}$ and the RE, or at least a very small moment if aligned AFM, and cause a negligible volume expansion of the unit cell. Possible mediators that can provide such a coupling include antiferromagnets, such as $\mathrm{Cr}$ and $\mathrm{Mn}$, and IEs, such as $\mathrm{N}$ and $\mathrm{P}$.

In a 2010 density functional theory study, Sanyal et ll $^{409}$ explored possible FM coupling of the TM and the $\mathrm{RE}$ via a Cr mediator, which couples AFM to both magnetic materials as illustrated in Fig. 26(a), and predicted large saturation magnetisations of the resulting stack as shown in Fig. 26(b). Suggested TMs (due to their already large moment and high Curie temperature) were $\mathrm{Fe}$ and $\mathrm{FeCo}$; and $\mathrm{Tb}, \mathrm{Gd}$, and Dy were proposed as their RE counterparts. The importance of growing the right crystal structure was highlighted, particularly concerning the $\mathrm{Cr}$ layer: ${ }^{410}$ only if $\mathrm{Cr}$ grows in a phase which allows a layer-wide collinear magnetic ordering of all atoms, it can work as an effective mediator between the adjacent $\mathrm{Fe}$ and $\mathrm{Gd}$ - thus promoting full FM coupling.

Converting the magnetic moment per atom values into magnetisation polarisation (using experimentally confirmed values rather than those from Hund's rule and assuming single-crystal structure) gives the saturation magnetisations

(a)
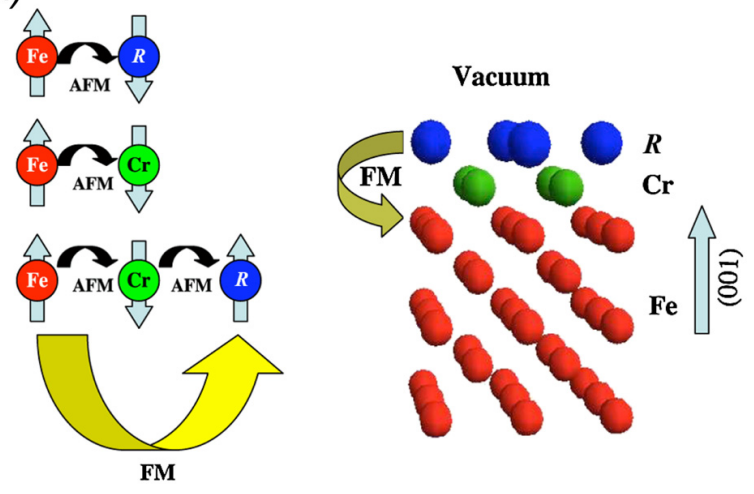

(b)

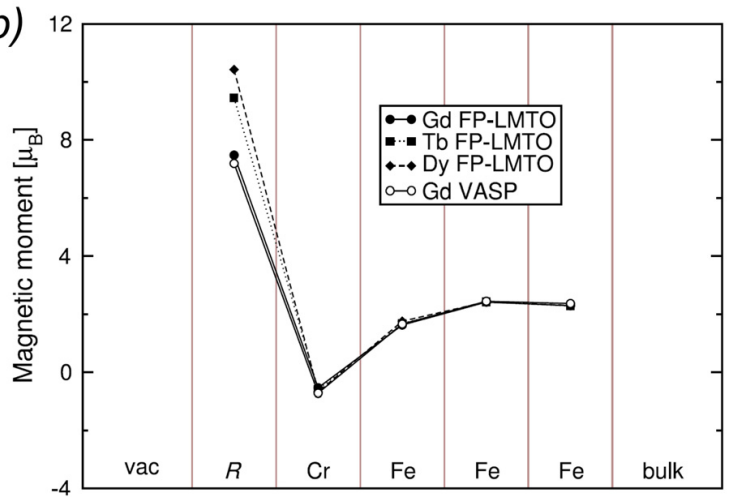

FIG. 26. (a) Conceptual sketch of how to achieve FM coupling between TMs and REs via a Cr spacer. (b) Predicted moment values for each ML of magnetic material in a $\mathrm{RE} / \mathrm{Cr} / \mathrm{Fe}$ trilayer stack according to density functional calculations. RE moment values are a superposition of calculated spin moments and atomic moments derived from Hund's rule. Graphs (a) and (b) are reprinted with permission from Sanyal et al., Phys. Rev. Lett. 104, 156402 (2010). Copyright 2010 the American Physical Society. ${ }^{409}$ 
TABLE III. Predicted saturation magnetisation values for FM coupling between a TM and a RE, mediated by an AFM Cr spacer (based on density functional calculations by Sanyal et al. ${ }^{409}$ ). Magnetic moments and atomic densities of the bulk equilibrium phases are assumed (in contrast to the original study in which atomic moments were derived via Hund's rule and hence tend to be rather large). Bold numbers indicate stack compositions with saturation magnetisation larger than the Slater-Pauling limit of $\mu_{0} M_{\mathrm{S}}=2.45 \mathrm{~T}$.

\begin{tabular}{|c|c|c|c|c|}
\hline$\mu_{0} M_{\mathrm{S}} / \mathrm{T}$ & $\begin{array}{c}\text { 1ML RE } \\
\text { 3ML Cr } \\
5 \mathrm{ML} \mathrm{TM}\end{array}$ & $\begin{array}{c}3 \mathrm{ML} \mathrm{RE} \\
5 \mathrm{ML} \mathrm{Cr} \\
7 \mathrm{ML} \mathrm{TM}\end{array}$ & $\begin{array}{c}3 \mathrm{ML} \mathrm{RE} \\
3 \mathrm{ML} \mathrm{Cr} \\
5 \mathrm{ML} \mathrm{TM}\end{array}$ & $\begin{array}{c}\text { 3ML RE } \\
\text { 3ML Cr } \\
\text { 7ML TM }\end{array}$ \\
\hline $\mathrm{Gd} / \mathrm{Cr} / \mathrm{Fe}$ & 1.58 & 1.71 & 1.89 & 1.93 \\
\hline $\mathrm{Gd} / \mathrm{Cr} / \mathrm{Fe}_{2} \mathrm{Co}$ & 1.71 & 1.81 & 1.98 & 2.04 \\
\hline $\mathrm{DyGd} / \mathrm{Cr} / \mathrm{Fe}$ & 1.70 & 1.91 & 2.15 & 2.15 \\
\hline $\mathrm{DyGd} / \mathrm{Cr} / \mathrm{Fe}_{2} \mathrm{Co}$ & 1.83 & 2.01 & 2.24 & 2.26 \\
\hline $\mathrm{Tb} / \mathrm{Cr} / \mathrm{Fe}$ & 1.75 & 2.00 & 2.26 & 2.25 \\
\hline $\mathrm{Tb} / \mathrm{Cr} / \mathrm{Fe}_{2} \mathrm{Co}$ & 1.88 & 2.10 & 2.36 & 2.37 \\
\hline $\mathrm{Dy} / \mathrm{Cr} / \mathrm{Fe}$ & 1.82 & 2.12 & 2.42 & 2.39 \\
\hline $\mathrm{Dy} / \mathrm{Cr} / \mathrm{Fe}_{2} \mathrm{Co}$ & 1.96 & 2.23 & 2.52 & 2.51 \\
\hline$\mu_{0} M_{\mathrm{S}} / \mathrm{T}$ & $\begin{array}{l}\text { 1ML RE } \\
1 \mathrm{ML} \mathrm{Cr} \\
5 \mathrm{ML} \mathrm{TM}\end{array}$ & $\begin{array}{c}\text { 1ML RE } \\
1 \mathrm{ML} \mathrm{Cr} \\
7 \mathrm{ML} \mathrm{TM}\end{array}$ & $\begin{array}{c}\text { 3ML RE } \\
1 \mathrm{ML} \mathrm{Cr} \\
7 \mathrm{ML} \mathrm{TM}\end{array}$ & $\begin{array}{c}\text { 3ML RE } \\
1 \mathrm{ML} \mathrm{Cr} \\
5 \mathrm{ML} \mathrm{TM}\end{array}$ \\
\hline $\mathrm{Gd} / \mathrm{Cr} / \mathrm{Fe}$ & 1.97 & 2.01 & 2.20 & 2.21 \\
\hline $\mathrm{Gd} / \mathrm{Cr} / \mathrm{Fe}_{2} \mathrm{Co}$ & 2.14 & 2.19 & 2.33 & 2.31 \\
\hline $\mathrm{DyGd} / \mathrm{Cr} / \mathrm{Fe}$ & 2.12 & 2.13 & 2.46 & 2.50 \\
\hline $\mathrm{DyGd} / \mathrm{Cr} / \mathrm{Fe}_{2} \mathrm{Co}$ & 2.29 & 2.31 & 2.59 & 2.61 \\
\hline $\mathrm{Tb} / \mathrm{Cr} / \mathrm{Fe}$ & 2.19 & 2.19 & 2.58 & 2.64 \\
\hline $\mathrm{Tb} / \mathrm{Cr} / \mathrm{Fe}_{2} \mathrm{Co}$ & 2.35 & 2.37 & 2.71 & 2.75 \\
\hline $\mathrm{Dy} / \mathrm{Cr} / \mathrm{Fe}$ & 2.28 & 2.26 & 2.74 & 2.83 \\
\hline $\mathrm{Dy} / \mathrm{Cr} / \mathrm{Fe}_{2} \mathrm{Co}$ & 2.45 & 2.44 & 2.87 & 2.94 \\
\hline
\end{tabular}

listed in Table III. In addition to the already suggested heavy RE metals, the $\mathrm{Dy}_{50} \mathrm{Gd}_{50}$ alloy was added. As apparent from Table III, a desirably high saturation magnetisation can only be achieved with RE metals having a higher moment than $\mathrm{Gd}$, such as $\mathrm{Tb}$ or Dy, preferably with $\mathrm{Fe}_{70} \mathrm{Co}_{30}$ being the TM. Since one ML of Cr always couples antiparallel to the remaining stack and its magnetic moment is a loss, only for thinnest $\mathrm{Cr}$ layers and high RE content reasonable magnetisation can be achieved.

Freeman and collaborators investigated Gd-N-Fe systems using all-electron density functional theory calculations including spin and orbital magnetic moments, as well as lattice relaxation. Starting with an $\mathrm{FeN}$ in a $\mathrm{NaCl}$ structure with AFM ordering, they replaced a central Fe atom in the cubic unit cell with Gd. This gave rise to a FM structure with a moment of $3.13 \mu_{B}$ per Fe atom but with a total magnetisation density corresponding to only $2.27 \mathrm{~T}$. They subsequently investigated GdN-Fe superlattices with one or two Gd-N planes ( $\mathrm{NaCl}$ structure) inserted into BCC Fe (see Fig. 27). However, it was found that these structures tend to be overall AFM. A couple of structures did exhibit a net FM magnetisation but with magnetisation densities below $2.10 \mathrm{~T} / \mu_{0}$.

Plenty more high-moment RE-TM combinations can be imagined, making use of a variety of IEs. A good starting point for considerations of such systems is $\mathrm{MnP}_{2}$. This compound crystallises in a hexagonal structure ${ }^{412}$ with two dissimilar Mn-sites, one with a small $\left(\approx 0.15 \mu_{B}\right)$ spin moment and the other with a much larger spin moment, about $2.78 \mu_{B}$, (a)

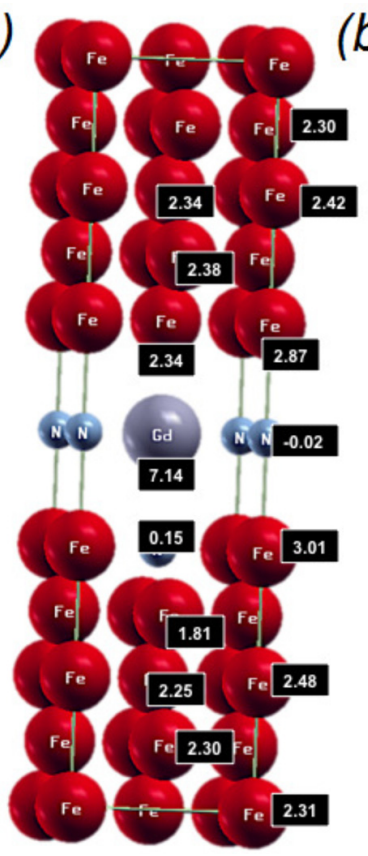

(b)

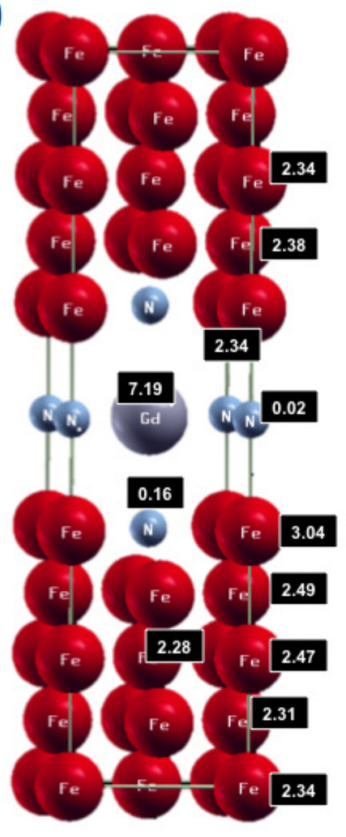

FIG. 27. Gd-N-Fe structures investigated by Freeman and co-workers ${ }^{411}$ obtained by inserting Gd-N layers into a BCC Fe structure. The structures in (a) and (b) were found to be FM but with total magnetisation densities of only $\mu_{0} M_{\mathrm{S}}^{(\mathrm{a})}=2.09 \mathrm{~T}$ and $\mu_{0} M_{\mathrm{S}}^{(\mathrm{b})}=2.02 \mathrm{~T}$. The numbers indicate the magnetic moment on each atom in units of $\mu_{B}$.

but the smaller P atoms promote an AFM coupling between the Mn atoms. ${ }^{413}$ One can substitute Fe for Mn and obtain a hexagonal FM structure where the two Fe sites have spin moments of 0.83 and $2.19 \mu_{B}$, respectively. While the saturation magnetisation density is not very high for $\mathrm{FeP}_{2}$ (corresponding to about only $1.03 \mathrm{~T}$ ), the $\mathrm{MnP}_{2}$ and $\mathrm{FeP}_{2}$ compounds nevertheless bring up the idea of using $\mathrm{P}$ as some interstitial "glue" between TM or RE atoms in more complex structures, where the RE couples similar to the IE. ${ }^{414}$ Model calculations of FeCoP, MnCoP, and MnFeP by Freeman and Heinonen ${ }^{411}$ showed that the $\mathrm{Mn}$ atoms could attain a large spin moment, up to $3.00 \mu_{B}$ in $\mathrm{MnFeP}$, but the moment reduction at the other TM site led to a low overall saturation magnetisation density (no more than $1.30 \mathrm{~T}$ ). One might try alleviate that loss of moment by replacing this TM with a high-moment RE. Indeed, initial tight-binding calculations ${ }^{411}$ indicated that GdMnP could attain a saturation magnetisation density of $\mu_{0} M_{\mathrm{S}}=2.73 \mathrm{~T}$ exceeding the maximum on the Slater-Pauling curve, with spin moments of 6.60 and $1.90 \mu_{B}$ on the $\mathrm{Gd}$ and $\mathrm{Mn}$ sites, respectively. However, the tightbinding calculations did not include volume expansion-such expansions typically take place when a large RE is inserted into some other host lattice, with the result that the net magnetisation density decreases substantially due to the volume expansion, while atomic spin and orbital moments can remain high. Indeed, structural optimisation using more sophisticated density functional-based methods resulted in a volume expansion of about $50 \%$ relative to the $\mathrm{MnP}_{2}$ mother compound with a net (spin) magnetisation of no more than $\mu_{0} M_{\mathrm{S}}=2.23 \mathrm{~T}$.

From all possible RE-TM systems which rely on mediating atoms, so far only MBE-grown $\mathrm{Gd} / \mathrm{Cr} / \mathrm{Fe}^{409,410,415}$ and 


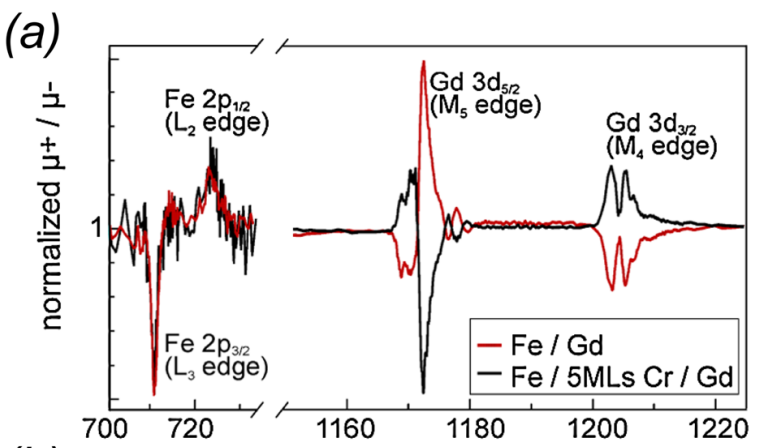

(b)

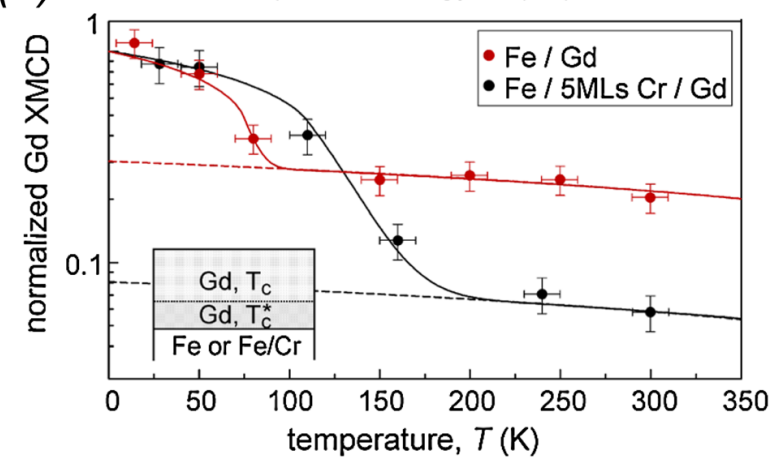

FIG. 28. (a) XMCD measurements showing the FM coupling of Gd and $\mathrm{Fe}$ in a $\mathrm{Gd} / \mathrm{Cr} / \mathrm{Fe}$ trilayer compared with an AFM coupling in a $\mathrm{Gd} / \mathrm{Fe}$ bilayer. (b) Temperature dependent XMCD scans suggesting increased Curie temperature for $\mathrm{Gd}$ in $\mathrm{Gd} / \mathrm{Cr} / \mathrm{Fe}$ assuming a higher $T_{\mathrm{C}}^{\star}$ for well-coupled $\mathrm{Gd}$ adjacent to the $\mathrm{Cr}$ and bulk $T_{\mathrm{C}}$ for the remaining Gd layer. Graphs (a) and (b) are reprinted with permission from Sanyal et al., Phys. Rev. Lett. 104, 156402 (2010). Copyright 2010 the American Physical Society. ${ }^{409}$

plasma-sputter-deposited $\mathrm{Gd} / \mathrm{Cr} / \mathrm{FeCo}^{351}$ have been investigated experimentally for their high-moment potential. Although FM coupling between those Gd and Fe MLs adjacent to the $\mathrm{Cr}$ spacer was proven by XMCD measurements $^{410}$ (shown in Fig. 28(a) in a comparison to an Fe/Gd bilayer), no high saturation magnetisation has been reported yet [see Fig. 29(a)]. XMCD measurements suggest there is an increase in the Curie temperature of those Gd MLs adjacent to the Cr spacer [see Fig. 28(b)], but there is no direct evidence so far. One reason for the reduced overall stack saturation magnetisation was the formation of FCC Gd for sputter-deposited films which suffers from reduced magnetisation $^{351}$ [see Fig. 29(b)]. A second reason might be the coupling behaviour of the RE to its adjacent capping or seed layer which has not been accounted for in the theoretical model. In a realistic system, the RE is not exposed to vacuum and requires protection from atmospheric $\mathrm{O}_{2}$ to prevent immediate oxidisation. Another general problem was the comparatively large thickness of the magnetic layers, which did not permit complete AFM coupling of the FM material to the $\mathrm{Cr}$ spacer, hence not fully mediating FM coupling between the RE and the TM and thus reducing the overall magnetisation of the stack.

The magnetic moments assumed for each layer are also open for debate. There are a number of reports on reduced $\mathrm{Fe}$ and $\mathrm{Gd}$ moments at $\mathrm{Cr}$ interfaces ${ }^{415}$ and, even worse, $\mathrm{Cr}$ was reported to have an increased moment at $\mathrm{Fe}$ interfaces, ${ }^{416-418}$ which would be a severe loss to the total
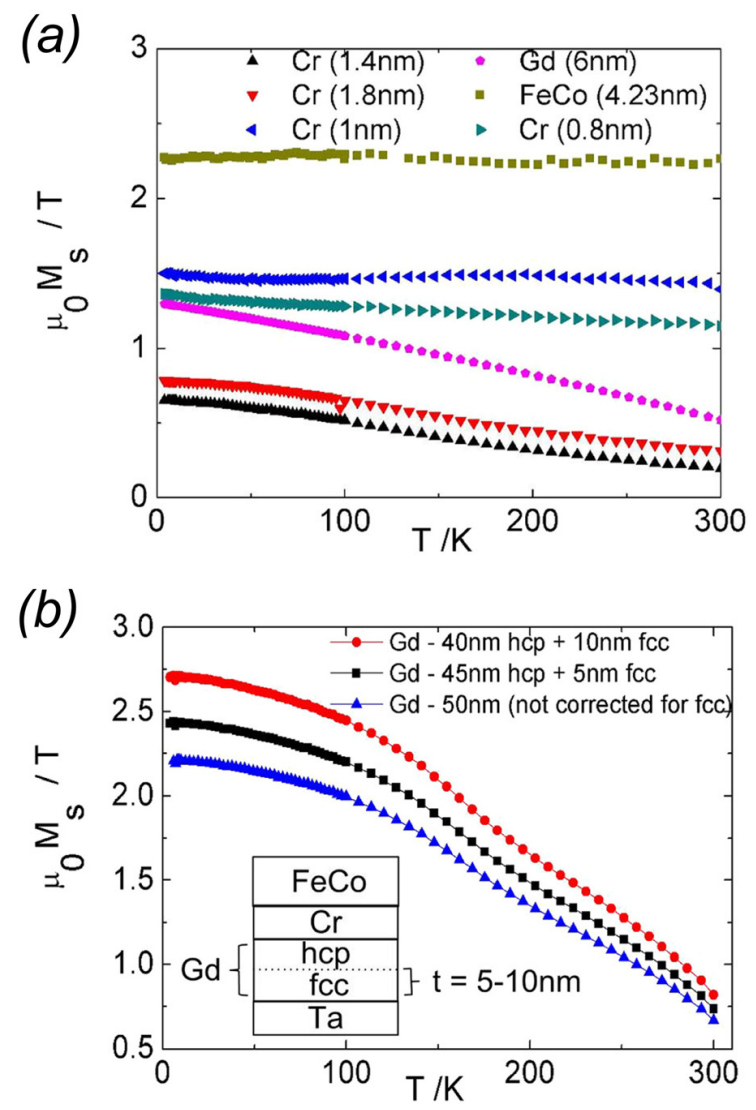

FIG. 29. (a) In-plane saturation magnetisation measurements of sputterdeposited $\mathrm{Gd} / \mathrm{Cr} / \mathrm{FeCo}$ trilayers. (b) Based on the findings of a considerable FCC phase with reduced magnetic moment at the seed interface of the Gd, the effective magnetic moment of an as-deposited $50 \mathrm{~nm}$ thick Gd film was effectively corrected for. Graphs (a) and (b) are reprinted with permission from Appl. Phys. Lett. 102, 092403 (2013). Copyright 2013 AIP Publishing LLC. ${ }^{351}$

magnetisation. An alternative material, serving the same purpose of FM coupling of REs and TMs by acting as an AFM mediator, could be Mn. It is known to couple antiparallel to other TMs such as Fe (see Section II D) and REs such as Gd (see Section IIG2). Beyond pure AFM mediators there is also the possibility to use alloys with similar magnetic properties, but perhaps more suitable crystal structure to achieve epitaxial match. Given the limitation of only up to three monolayers of spacer material in the stack for optimal coupling, a Cr or Mn alloy could be chosen which additionally promotes improved texture of the following magnetic material.

It is worth noting that $\mathrm{Cr}$ does not necessarily couple fully AFM to all heavy RE metals; however experimental evidence indicates AFM coupling for $\mathrm{Gd} / \mathrm{Cr}$ bilayers ${ }^{419}$ and multilayers. ${ }^{420}$ Furthermore, there are sporadic reports of AFM coupling of the surface layer of $\mathrm{Gd}$ (and $\mathrm{Tb}$ ) to the bulk-with a similar number of studies reporting FM coupling. ${ }^{421}$ Nevertheless, this phenomenon might explain the generally low magnetisation of $\mathrm{Re} / \mathrm{Cr} / \mathrm{TM}$ trilayer stacks: even though there is clearly antiparallel coupling of $\mathrm{Gd}$ and $\mathrm{Cr}$ at the interface (and thus effectively parallel coupling of the interface Gd layer to the Fe film), the bulk of the Gd film couples antiparallel to the $\mathrm{Fe}$ (or $\mathrm{FeCo}$ ) film. 


\section{Summary: Combined RE-TM systems}

So far, there is no confirmed report of a magnetic moment, and hence saturation magnetisation gain for a combined RE-TM system. Coey $^{35}$ summarised the theoretical understanding, which supports observations on alloy systems that have been investigated so far: "Exchange coupling between atoms with $d$ shells that are more than half full with atoms whose $d$ shell is less than half full is antiferromagnetic. The rare-earths in this context should be considered as light $d$ elements because (of) their atomic configuration (...). There is, therefore, antiparallel coupling of the spin moments of the ferromagnetic $3 d$ elements $\mathrm{T}=\mathrm{Fe}, \mathrm{Co}$, and $\mathrm{Ni}$, and the spin moment of a rare-earth metal. (...) This leads to antiparallel coupling of the atomic moments in R-T alloys with $\mathrm{R}=\mathrm{Gd}-\mathrm{Yb}$." RE-Mn alloys, thought to couple FM in $\mathrm{RE}_{6} \mathrm{Mn}_{23}$, are in fact FIM. The RE-Mn system stabilised by small IEs might be worth exploring, starting from the GdMnP compound with a predicted best-case magnetisation of $\mu_{0} M_{\mathrm{S}}=2.73 \mathrm{~T}$.

Experimental results on $\mathrm{RE} / \mathrm{Cr} / \mathrm{TM}$ ultrathin trilayer stacks have not shown any moment improvement either. However, first experimental investigations in the $\mathrm{Gd} / \mathrm{Cr} / \mathrm{Fe}$ system showed clear indication of parallel coupling of the $\mathrm{RE}$ and the TM in proximity to the $\mathrm{Cr}$ spacing layer, which is a promising starting point for further research. A theoretical study on the magnetic properties of a RE-Cr-TM alloy would offer insight, whether or not the idea of using $\mathrm{Cr}$ (or $\mathrm{Mn}$ ) as a mediator for FM coupling could also work in a ternary alloy.

\section{H. A remark on TM and RE clusters and nanoparticles}

The behaviour of TM clusters and nanoparticles is best depicted at the example of Fe. Similar to reduceddimension ultrathin films of $\mathrm{Fe}$, theory also predicts a moment increase and $T_{\mathrm{C}}$ decrease for nanoparticles and free clusters of $\mathrm{Fe}^{422-424}$ and $\mathrm{FeCo} .{ }^{425}$ Numerous experimental studies have confirmed those predictions for free clusters and nanogranular compounds, ${ }^{426-429}$ and also clusters embedded in NM matrices were shown to have increased magnetic moments. ${ }^{426,430,431}$ On the contrary, there are also reports of TM nanoparticles with reduced moment, possibly as a result of oxidation. ${ }^{432-435}$

For RE metals, certain MLs in surface proximity were predicted to have increased moments ${ }^{436}$ which might be harnessed in clusters. There is literally no literature available on the saturation magnetisation of pure RE clusters or nanoparticles, which is primarily due to fabrication and handling issues resulting from high $\mathrm{RE}$ reactivity which gets even worse for nanostructures with increased surface-to-volume ratio. ${ }^{437}$ Review articles by Bucher et al. ${ }^{438}$ and Bansmann et $a l .{ }^{439}$ indicate magnetic moments well below bulk values. An approach to bypass handling problems is to compact nanoparticles into a tight ensemble in-situ; however, coupling between the grains impacts on the magnetic properties. Magnetic moments were also found to drop for those nanogranular compounds-examples are studies on Gd by Yue et al. $^{440}$ and on Gd, Dy, Tb by O'Shea and Perera. ${ }^{441}$ Most attempts in synthesising pure RE nanoparticles resulted in crystallites with severe lattice stretch often ordering directly in the low-moment FCC phase-a phenomenon which is known as "size-induced structural phase transformation.",442 Therefore, even if corrosion can be avoided, it appears highly unlikely that free high-moment RE metal nanoparticles can ever be synthesised. An alternative route would be to embed RE particles in an appropriate matrix or to cover them in a shell which helps stabilising the equilibrium high-moment HCP phase. For either scenario, there is no confirmed report of a moment increase so far, but rather the opposite as for $\mathrm{Gd}$, Dy, and $\mathrm{Tb}$ nanoparticles in a $\mathrm{Ti}$ matrix. ${ }^{441,443}$

A general problem for all nanostructures is posed by the conflicting relationship of magnetic moment and the SPM limit. Smaller particles or clusters do benefit from surfaceenhanced magnetic moments or, in the case of TMs, also moment enhancement due to a lattice stretch (comparable to ultrathin films). But smaller particles are also more prone to thermal instability of their magnetisation. For TMs, only Fe clusters in an NM matrix were shown to keep their FM state at higher temperatures, having a less severe moment drop with increasing temperature. A common problem for all magnetic nanostructures, but particularly pronounced for RE metals, is $\mathrm{O}_{2}$ diffusion and resulting degradation of the magnetic properties. A very comprehensive review, listing magnetic moments and transition temperatures of various nanocluster systems, is given by Bansmann et al. ${ }^{439}$

\section{SUMMARY}

The limit for saturation magnetisation of bulk materials has not changed for decades now: at cryogenic temperatures pure $4 f$-based RE metals still hold the lead and at room temperature FeCo sets the unwavering standard. Thin films ( $\approx 100 \mathrm{~nm}$ layer thickness) were shown to roughly follow bulk guidelines. However, they often suffer from reduced magnetisation due to granularity issues ${ }^{444,445}$ or, as for heavy RE metals, from strain ${ }^{165}$ and only keep bulk magnetisation for epitaxial films of equal crystal structure. Ultrathin films $(t<2.5 \mathrm{~nm})$ of TMs benefit from surface-enhanced magnetic moments or the stabilisation of an otherwise metastable crystal structure; however, their easy axis direction often moves out-of-plane due to the growing influence of surface anisotropy. In general, the magnetic moment of TM atoms is maximal if they are isolated and therefore the magnetic moment in a crystal increases if the nearest-neighbour distance is increased. This can be achieved for instance by a lattice stretch of an Fe crystallite, ${ }^{431}$ effectively turning the Fe into a high-moment strong ferromagnet. But this stretch has, of course, its limits for applications which require large saturation magnetisation, i.e., a large magnetic moment per unit volume. Ultrathin films of RE metals seem to keep their high bulk moment at cryogenic temperatures, given epitaxial layer quality. But on most substrates ultrathin RE metals order FCC with a significantly stretched lattice compared to the equilibrium HCP phase, ${ }^{348}$ with drastically smaller magnetic moment and saturation magnetisation than for singlecrystal specimen. ${ }^{165}$ Magnetically dead layers at interfaces due to hybridisation are a problem particularly in laminates 
for TMs and REs alike, however much more challenging for the latter since practically no moment conserving substrate or universal seedlayer material is known.

All high-saturation-magnetisation candidates listed in this review can be classified into the six most relevant categories below, whereas the standard was set to be bulk $\mathrm{Fe}_{65-70} \mathrm{Co}_{35-30}$ with $\mu_{0} M_{\mathrm{S}}=2.45 \mathrm{~T}$. Confirmed materials have a broad literature base and were repeatedly shown to have their large saturation magnetisation. Potential material systems were either only theoretically predicted, their high moment was only reported in isolated studies or there are conflicting moment values. Unsuitable materials cannot be used for write pole tips or similar applications which require large saturation magnetisations. The classification bulk includes thin films of thickness $>50 \mathrm{~nm}$ (although their magnetisation is mostly a bit less than the bulk due to finite size effects).

\section{Confirmed-bulk, room temperature}

- $\mathrm{Fe}_{65-70} \mathrm{Co}_{35-30}\left(\mu_{0} M_{\mathrm{S}}=2.45 \mathrm{~T}\right)$

2. Potential-bulk, room temperature

- $\mathrm{Fe}_{16} \mathrm{~N}_{2}\left[\mu_{0} M_{\mathrm{S}}=2.9 \mathrm{~T}\right.$; although experimental reports so far indicate only $\mu_{0} M_{\mathrm{S}} \approx 2.3 \mathrm{~T}$ (Ref. 32)]

- $\left(\mathrm{Fe}_{70} \mathrm{Co}_{30}\right)_{98.96} \mathrm{Pd}_{1.04}\left(\mu_{0} M_{\mathrm{S}}=2.49 \mathrm{~T}\right)$

- TM-X-RE alloys (X=Cr, Mn, IE); e.g., GdMnP $\left(\mu_{0} M_{\mathrm{S}} \leq 2.73 \mathrm{~T}\right)$

3. Confirmed-bulk, cryogenic temperatures

- Ho $\left(\mu_{0} M_{\mathrm{S}}=3.87 \mathrm{~T}\right)$

- Dy $\left(\mu_{0} M_{\mathrm{S}}=3.82 \mathrm{~T}\right)$

- $\mathrm{Tb}\left(\mu_{0} M_{\mathrm{S}}=3.40 \mathrm{~T}\right)$

- $\operatorname{Gd}\left(\mu_{0} M_{\mathrm{S}}=2.66 \mathrm{~T}\right)$

4. Potential-ultrathin films, cryogenic temperatures

- $\mathrm{Fe} / \mathrm{NM}(\mathrm{NM}=\mathrm{Au}, \mathrm{Ag})\left(\mu_{0} M_{\mathrm{S}}=2.6 \mathrm{~T}\right)$

- $\mathrm{Fe} / \mathrm{Pd}\left(\mu_{0} M_{\mathrm{S}}=2.7 \mathrm{~T}\right)$

- $\mathrm{Fe}_{16} \mathrm{~N}_{2}\left(\mu_{0} M_{\mathrm{S}}=3.2 \mathrm{~T}\right)$

- Ho, Dy, Tb, Gd (if unstrained and of epitaxial quality-high moment only for HCP equilibrium phase)

- TM/X/RE trilayers (X=Cr, Mn, IE); e.g., 3ML Dy/ $1 \mathrm{ML} \mathrm{Cr} / 5 \mathrm{ML} \mathrm{Fe}_{70} \mathrm{Co}_{30}\left(\mu_{0} M_{\mathrm{S}}=2.94 \mathrm{~T}\right)$

5. Potential-ultrathin films, room temperature

- $\mathrm{C} / \mathrm{Fe}_{70} \mathrm{Co}_{30} / \mathrm{Au}$ trilayers $\left(\mu_{0} M_{\mathrm{S}}=2.70 \mathrm{~T}\right)$

- $\mathrm{Fe}_{70} \mathrm{Co}_{30} / \mathrm{Pd}$ as in laminates $\left(\mu_{0} M_{\mathrm{S}}=2.57 \mathrm{~T}\right)$

- $\mathrm{Fe}_{16} \mathrm{~N}_{2}\left(\mu_{0} M_{\mathrm{S}}=2.9 \mathrm{~T}\right)$

- TM/X/RE trilayers $(\mathrm{X}=\mathrm{Cr}, \mathrm{Mn}, \mathrm{IE})$; e.g., 3ML Dy/ $1 \mathrm{ML} \mathrm{Cr} / 5 \mathrm{ML} \mathrm{Fe}_{70} \mathrm{Co}_{30}\left(\mu_{0} M_{\mathrm{S}}<2.94 \mathrm{~T}\right)$

6. Unsuitable

- Fe-NM (NM = Au, Ag, Pt, Pd, Rh) and FeSc alloys

- MnFe binary alloys

- RE-TM binary alloys (including RE-Mn alloys)

- RE-NM (NM = Au, Ag, Pt, Pd, Rh) and RE-Sc alloys

- direct RE-TM laminates

- Gd/W laminates

- Gd-GaN compounds

In general, high saturation magnetisation comes at the expense of softness as well as initial permeability-both of which are of pivotal importance for solenoid pole pieces operating at high frequencies. This might be referred to as the classic "dilemma" of soft magnetic materials (in reference to the trilemma of PMR). This trend does not exclusively apply to $3 d$ TMs, whose coercivity increases with increasing saturation magnetisation while the initial permeability decreases, which is shown in Figs. 30(a) and 30(b), respectively.

But a similar tendency can also be observed for elemental RE metals: those with high saturation magnetisation are also the hardest and suffer from lowest initial permeability, as depicted in Fig. 31 at the examples of Gd, Tb, and Dy thin films. A main contributor to RE coercivity, however, comes from polycrystalline ordering and the coercivity of single crystals of $\mathrm{Gd}, \mathrm{Tb}$, and Dy (measured in the easy-axis direction) is at least one order of magnitude smaller than the here presented thin films. ${ }^{327,447}$ Continually improving the layer quality, to single-crystal level if possible, will hence likely directly lead to a further reduction of the coercivity. ${ }^{165}$ Softness of hard high-moment RE metals such as Dy can also be improved by adding small quantities of other RE metals with favourable crystal anisotropy, or using those as seed materials, but this comes at the expense of magnetic moment. This is also in analogy to the TM case: $\mathrm{Fe}_{65} \mathrm{Co}_{35}$ can be softened by adding $\mathrm{Ni}$ or seeding it with permalloy, but the saturation magnetisation is reduced [see Fig. 6(a)].

When pursuing a general increase in the magnetic moment of TMs, not necessarily aiming for large saturation, the neighbours of choice are NMs, particularly $\mathrm{Pd}$. This holds true for magnetic TMs diluted not only in an alloy but also in the thin-film stacks. For REs, only other RE metals (or quasi-REs) with very similar electronic structure assure moment conservation, such as in the case of $\mathrm{Y}$ and Gd. Fe and Fe-rich compounds show no signs of a deteriorating magnetic moment in proximity to GaAs, which makes it a useful substrate for high-moment applications.

Utilising the moment increase of FM materials at lower temperatures by actively cooling the pole tip is limited to macroscopic devices: there is no viable cooling method available at the present time for their micro- or nanoscale counterparts as in HDD write heads.

\section{OUTLOOK}

It is a most remarkable curiosity that, even after more than a century of research and device miniaturisation penetrating the nanoregime, FeCo alloys are still the number one choice for pole pieces in electromagnets operating at room temperature such as in HDD write heads. The unrivalled saturation magnetisation of bulk $\mathrm{Fe}_{65-70} \mathrm{Co}_{35-30}$, in conjunction with secondary magnetic properties like softness, high initial permeability, as well as crystal anisotropy and magnetostriction which can be comfortably tuned by alloying or multilayering, makes it the champion among a huge variety of high-moment material candidates.

Thin films of $\alpha^{\prime \prime} \mathrm{Fe}_{16} \mathrm{~N}_{2}$ have shown their potential to replace $\mathrm{Fe}_{2} \mathrm{Co}$ considering their saturation magnetisation, however only MBE-synthesis succeeded, which is unsuitable for large-scale industrial fabrication, and the $\alpha^{\prime \prime}$ phase was reported to decompose dangerously close to room temperature. In addition, the delicate $\alpha^{\prime \prime}$ phase has comparatively large anisotropy, which will pose a serious challenge for 


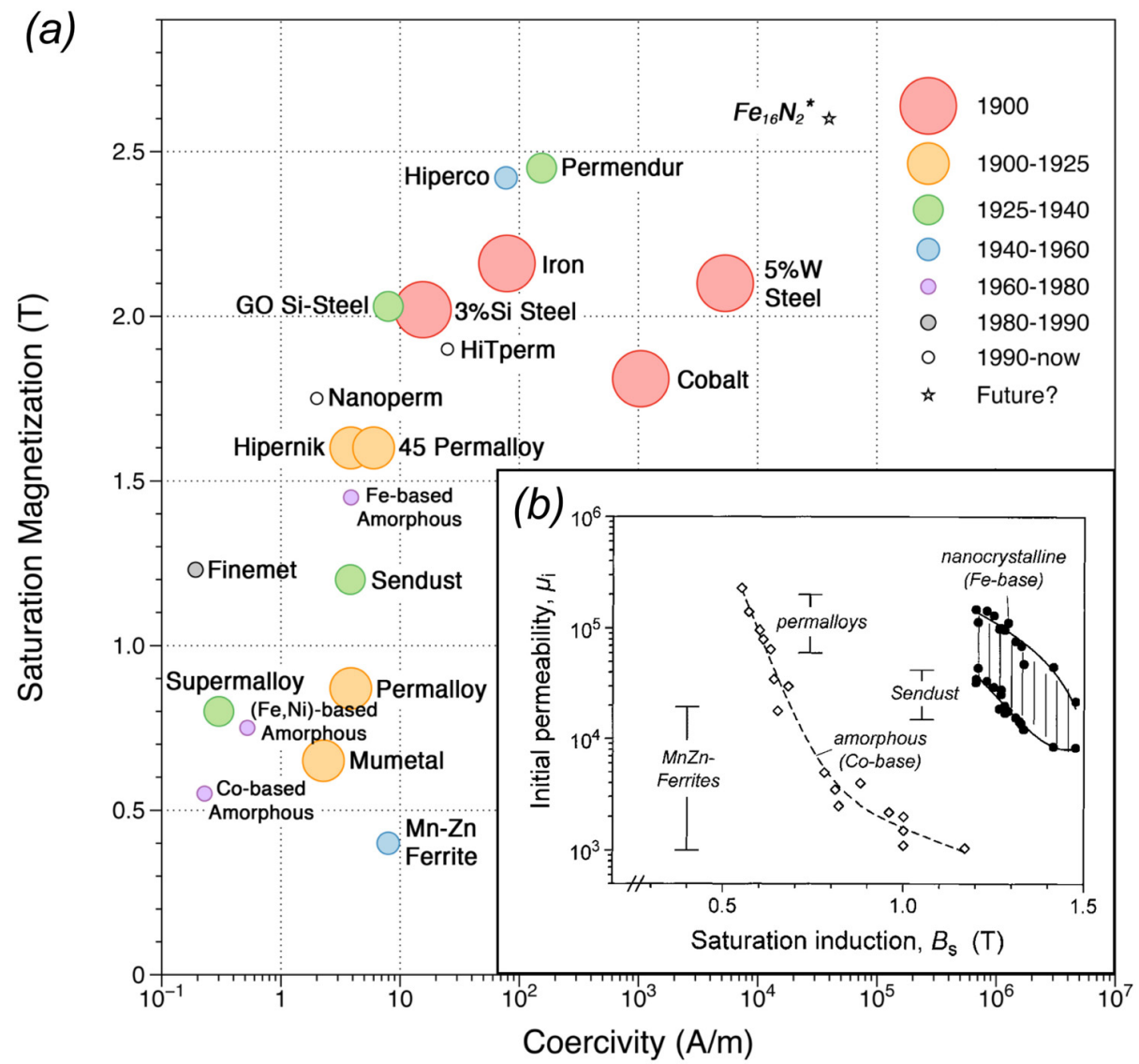

FIG. 30. The classic "dilemma" of soft magnetic materials: high saturation magnetisation of reasonably soft TMs is concomitant with (a) large coercivity $H_{\mathrm{C}}$ and (b) low initial permeability $\mu_{\mathrm{i}}$. Graph (a) from Frontiers of Engineering: Reports on Leading-Edge Engineering. Copyright 2013 National Academy of Science. Reprinted with permission from National Academies Press. ${ }^{318}$ Graph (b) from Herzer, Handbook of Magnetic Materials. Copyright 1997 Elsevier. Reprinted with permission from Elsevier. ${ }^{446}$
$\mathrm{GHz}$ operating frequency applications, if it can be reliably synthesised and integrated in a recording head at all.

With increasing miniaturisation and write poles shrinking, there is a growing and justified interest in epitaxially grown ultrathin film systems with a thickness below $t_{\mathrm{c}}=2.5 \mathrm{~nm}$. Those hold the potential for cracking the SlaterPauling limit by resorting to interface-improved magnetic moments (Fe/NM, FeCo/Pd), tailored metastable crystal structures ( $\mathrm{FeCo}, \alpha^{\prime \prime} \mathrm{Fe}_{16} \mathrm{~N}_{2}$ ) or FM coupling of TMs and REs via ML-thin AFM spacers such as in $\mathrm{Dy} / \mathrm{Cr} / \mathrm{Fe}_{70} \mathrm{Co}_{30}$ or $\mathrm{Tb} / \mathrm{Cr} / \mathrm{Fe}_{70} \mathrm{Co}_{30}$ (of the general group of $\mathrm{RE} / \mathrm{Cr} / \mathrm{TM}$ ). There is large ambiguity about the magnetisation vs. temperature profile of these ultrathin films as well as their magnetic anisotropy and saturation behaviour and more research needs to be done to identify points of magnetic transition. So far, only $\mathrm{Fe}_{70} \mathrm{Co}_{30}$ ultrathin films, $\mathrm{Fe}_{70} \mathrm{Co}_{30} / \mathrm{Pd}$ laminates, and $\mathrm{Fe}_{16} \mathrm{~N}_{2}$ seem to exhibit their large saturation at room temperature, but the number of experimental studies is very limited and reported moment gains are often well within the uncertainty range - or contradicted by different studies. Due to the small sample volume and difficult indirect structural analysis, determining the actual saturation magnetisation of ultrathin films still poses a serious challenge. Although the idea to search for a material with large magnetisation within the larger group of Heusler alloys is voiced occasionally, a huge base of experimental results as well as theoretical understanding so far strongly argue against this concept.

Replacing the FeCo write pole tip with a RE metal such as $\mathrm{Gd}, \mathrm{Tb}$, Dy and their alloys is indeed an alternative route to bypass the Slater-Pauling limit, given sufficient corrosion protection and cooling. However, experimental results on the magnetisation behaviour of RE films with thickness in the relevant range, which show polycrystalline ordering, raise concerns about their softness, which is a requirement for write pole application to assure a quick writing process. Although in principle useful considering their saturation magnetisation at cryogenic temperatures (see Fig. 21), these films exceed the magnetisation of much softer FeCo alloys only for large applied fields of $\mu_{0} H>2 \mathrm{~T}$ (at $T \rightarrow 0 \mathrm{~K}$ ), beyond the reach of $\mathrm{Cu}$-based solenoids even when employing a tiered flux concentrator, which limits their applicability to superconducting solenoids. It was suggested to alloy elemental RE metals with other REs to compensate the anisotropies of different crystallites and hence to acquire a softer material, such as in HoSm or DyEr. This approach has to be pursued with caution since some (polycrystalline) inter-RE alloys with promising thermo-magnetic profiles, such as DyTb, were reported to have huge magnetostriction. In a pole tip scenario, this might have a drastically negative effect on the write field gradient (worse than in the case of TMs) and would thus become an additional severe obstacle for application in thinfilm solenoids. The synthesis and analysis of single-crystal quality epitaxial thin films of (elemental) RE metals could shed some light on possible further use as write pole tips, since these films are expected to be much softer and hence to actually saturate in moderate applied fields.

More exotic candidates to crack the Slater-Pauling limit are among the RE-TM alloys. Due to the AFM coupling 


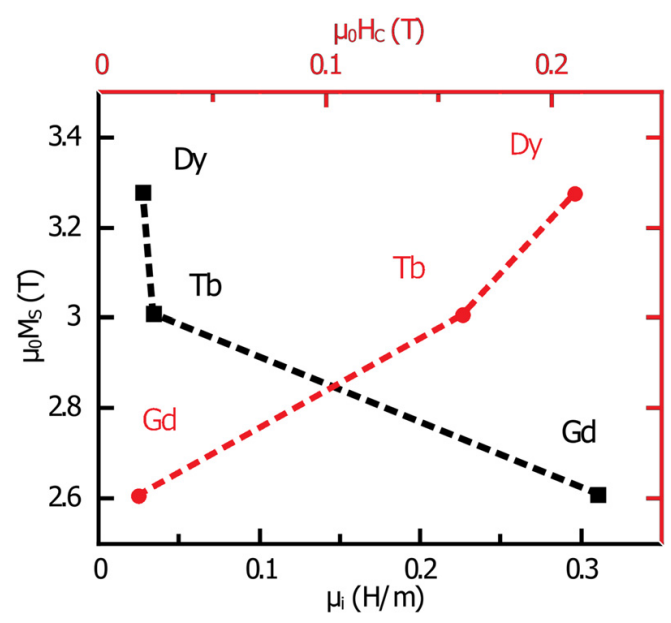

FIG. 31. As for TMs, high saturation magnetisation of elemental RE metals is concomitant with large coercivity $H_{\mathrm{C}}$ (red dots) and low initial permeability $\mu_{\mathrm{i}}$ (black squares). Note, the data is based on in-plane SQUID measurements at $T=4 \mathrm{~K}\left(\mu_{0} H_{0}=0.01 \mathrm{~T}\right)$ of polycrystalline yet strongly $\left(\begin{array}{lll}0 & 0 & 2\end{array}\right)$ textured thin films of $\mathrm{Gd}, \mathrm{Tb}$, and $\mathrm{Dy}$ in $\mathrm{SiO}_{2}: \mathrm{Ta}(5 \mathrm{~nm}) / \mathrm{RE}(50 \mathrm{~nm}) / \mathrm{Ta}(5 \mathrm{~nm})$ trilayer stacks as in Ref. 165.

of RE metals and TMs, if in direct contact, IEs might be a solution to adjust the interatomic spacing accordingly (REIE-TM), for instance in GdMnP. Going a step further and creating ternary alloys using mediating elements such as $\mathrm{Cr}$, $\mathrm{Mn}$, or their respective alloys, which are know to couple AFM to heavy RE metals and FeCo, might also result in a high-moment material with large operating temperature. However, the suggested three-component materials lack any experimental evidence.

In the context of HAMR technology, magnetic properties of the pole piece material, which are considered less important than saturation moment for conventional PMR systems, might be of relevance again. The requirement of precise switching in the Hopkinson peak of the recording medium can best be served by write pole materials which have a large Curie temperature, are soft and have large initial permeability. Elemental RE metals, however, are hardly suitable, since they are highly temperature sensitive and also comparatively hard. Among the TMs previous pole piece materials might again be of interest, not only for their much higher initial permeability but also for better corrosion resistance at higher temperatures and lower magnetostriction compared to pure $\mathrm{Fe}_{65-70} \mathrm{Co}_{30-35}$.

The following list gives some examples of soft amorphous or nanocrystalline TM alloys and compounds which might serve as HAMR pole-piece materials, having sufficiently large $T_{\mathrm{C}}>700 \mathrm{~K}$ and thermal stability: $:^{1,446,448}$

- permalloy $\left(\mathrm{Ni}_{80} \mathrm{Fe}_{20}\right)^{449}$ or supermalloy $\left(\mathrm{Ni}_{78} \mathrm{Fe}_{17} \mathrm{Mo}_{5},{ }^{98}\right.$ $\left.\mu_{0} M_{\mathrm{S}} \approx 0.7 \mathrm{~T}\right)$

- permalloy-FeCoN laminates ${ }^{143}\left(\mu_{0} M_{\mathrm{S}} \approx 2.4 \mathrm{~T}\right)$

- FeCo: $\mathrm{Ni}^{450}$ or FeCo:Pd ${ }^{56}\left(\mu_{0} M_{\mathrm{S}} \approx 2.4 \mathrm{~T}\right)$

- hitperm $\left[\left(\mathrm{Fe}_{70} \mathrm{Co}_{30}\right)_{88} \mathrm{Zr}_{7} \mathrm{~B}_{4} \mathrm{Cu}_{1},{ }^{451,452} \mu_{0} M_{\mathrm{S}}=1.9 \mathrm{~T}\right]$

- metglas $\left(\mathrm{Fe}_{67} \mathrm{Co}_{18} \mathrm{~B}_{14} \mathrm{Si}_{1},{ }^{98} \mu_{0} M_{\mathrm{S}}=1.8 \mathrm{~T}\right)$

- $\mathrm{Fe}_{50} \mathrm{Co}_{50}$ (softened by IE such as in $\mathrm{Co}_{40} \mathrm{Fe}_{40} \mathrm{~B}_{20}-\mathrm{SiO}_{2}{ }^{453}$ $\left.\mu_{0} M_{\mathrm{S}}=2.4 \mathrm{~T}\right)$

- sendust $\left[\mathrm{Fe}_{85} \mathrm{Si}_{9} \mathrm{Al}_{6}, \mu_{0} M_{\mathrm{S}}=1.0 \mathrm{~T}\right.$ (Ref. 2)]
- FeAlN (e.g., in laminates with $\mathrm{Al}_{2} \mathrm{O}_{3},{ }^{454} \mu_{0} M_{\mathrm{S}} \approx 1.9 \mathrm{~T}$ )

- finemet $\left(\mathrm{Fe}_{73.5} \mathrm{Si}_{13.5} \mathrm{Nb}_{3} \mathrm{~B}_{9} \mathrm{Cu}_{1},{ }^{98} \mu_{0} M_{\mathrm{S}} \approx 1.3 \mathrm{~T}\right)$

- CoZr alloys (e.g., CoZrTa softened by laminating with $\mathrm{SiO}_{2},{ }^{455}$ and $\mathrm{CoNbZr}$ softened by Tb-doping, ${ }^{456}$ $\left.\mu_{0} M_{\mathrm{S}} \approx 1.4 \mathrm{~T}\right)$

It is important to highlight that actual device integration requires thin films - and particularly nanostructures-being fabricated in sufficient quality (i.e., purity, smoothness, crystallinity, long-term stability, and compatibility to adjacent materials). This needs to be evaluated for any potential $\mathrm{Fe}_{65-70} \mathrm{Co}_{30-35}$ alternative individually. The impact of miniaturisation (down to the nanometre regime) on the magnetic properties is not a trivial prediction and becomes even more difficult for $\mathrm{GHz}$ operation and faster.

Although not the main concern of this review, we hope to raise interest in some of the more exotic and perhaps not immediately applicable material systems we introduced. For instance new SC-TM combinations, i.e., a SC seed material tailored to conserve the moment of $\mathrm{Ni}$ or $\mathrm{Co}$ rather than $\mathrm{Fe}$, resorting to group III-V combinations other than GaAs, InAs, and $\mathrm{GaP}$ or perhaps candidates drawn from the II-II-V or II-VI pool-currently of great interest for diluted magnetic SCs for spintronic applications. ${ }^{457}$ A deeper understanding of the Gd-GaN and similar material systems might pave the way for more targeted research on (group III-V) SCs as substrates for conserving or even increasing the magnetic moment of RE metals such as Tb or Dy, for whom suitable seed layer combinations are still unknown. And understanding the origin of softness and what seems to be FM coupling in DyRhNi might help to identify new solutions for achieving parallel RE-TM coupling in alloys or laminates.

\section{ACKNOWLEDGMENTS}

The authors would like to thank M. Coey (Trinity College, Dublin) and R. Chantrell (University of York) for helpful discussions and D. Oron (Weizmann Institute of Science) for his ongoing support in publishing this review. Special thanks go to A. Freeman (Northwestern University) for first-principle work on the RE-IE-TM system, which helped identifying $\mathrm{GdMnP}$ as a possible high-moment candidate. We would also like to thank Seagate Technology (Ireland) for financial support to establish ANSIN (www.ansin.eu). The work by O. Heinonen was supported by the Department of Energy, Office of Science, Materials Science and Engineering Division.

${ }^{1}$ K. H. J. Buschow and F. R. de Boer, Physics of Magnetism and Magnetic Materials (Kluwer Academic Publishers, New York, Boston, Dordrecht, London, Moscow, 2003).

${ }^{2}$ M. H. Kryder, "An introduction to magnetic recording heads," in Magnetic Storage Systems Beyond 2000, NATO Science Series, edited by G. C. Hadjipanayis (Kluwer Academic Publishers, Dordrecht, Netherlands, 2001), Vol. 41; available at http://www.avc.com.hr/eurostar/Teh_Podrska/ DVR/DVRdocuments/An\%20Introduction\%20to\%20Magnetic\%20 Recording $\% 20$ Heads.pdf.

${ }^{3}$ E. I. Cooper, C. Bonhote, J. Heidmann, Y. Hsu, P. Kern, J. W. Lam, M. Ramasubramanian, N. Robertson, L. T. Romankiw, and H. Xu, "Recent developments in high-moment electroplated materials for recording heads," IBM J. Res. Dev. 49(1), 103-126 (2005). 
${ }^{4}$ K. Noma, M. Matsuoka, H. Kanai, Y. Uehara, K. Nomura, and N. Awaji, "Ultra-high magnetic moment films for write head," IEEE Trans. Magn. 42(2), 140 (2006).

${ }^{5}$ T. Coughlin, See http://www.forbes.com/sites/tomcoughlin/2015/01/29/ hdd-annual-unit-shipments-increase-in-2014/ for "HDD Annual Unit Shipments Increase In 2014" (Forbes online), 2015.

${ }^{6}$ Y. Shiroishi, K. Fukuda, I. Tagawa, H. Iwasaki, S. Takenoiri, H. Tanaka, H. Mutoh, and N. Yoshikawa, "Future options for HDD storage," IEEE Trans. Magn. 45(10), 3816 (2009).

${ }^{7}$ A. Michael, V. Thierry, M. Terry, and B. Charles, "Optically-assisted magnetic recording,” J. Magn. Soc. Jpn. 25, 328-333 (2001).

${ }^{8}$ T. Rausch, P. Herget, J. A. Bain, J. Zhu, D. D. Stancil, and T. E. Schlesinger, "Experimental test bed for hybrid recording," SPIE Proc. 4342, 502-510 (2002).

${ }^{9}$ T. W. McDaniel, "Ultimate limits to thermally assisted magnetic recording,” J. Phys.: Conden. Matter 17, R315 (2005).

${ }^{10}$ R. Rottmayer, S. Batra, D. Buechel, W. Challener, J. Hohlfeld, Y. Kubota, L. Li, B. Lu, C. Mihalcea, K. Mountfield, K. Pelhos, C. Peng, T. Rausch, M. A. Seigler, D. Weller, and X. Yang, "Heat-assisted magnetic recording," IEEE Trans. Magn. 42, 2417-2421 (2006).

${ }^{11}$ M. Kryder, E. Gage, T. McDaniel, W. Challener, R. Rottmayer, G. Ju, Y.-T. Hsia, and M. Erden, "Heat assisted magnetic recording," Proc. IEEE 96, 1810-1835 (2008).

${ }^{12}$ M. A. Seigler, W. Challener, E. Gage, N. Gokemeijer, G. Ju, B. Lu, K. Pelhos, C. Peng, R. Rottmayer, X. Yang, H. Zhou, and T. Rausch, "Integrated heat assisted magnetic recording head: Design and recording demonstration," IEEE Trans. Magn. 44, 119-124 (2008).

${ }^{13}$ R. L. Stamps, S. Breitkreutz, J. Ảkerman, A. V. Chumak, Y. Otani, G. E. W. Bauer, J.-U. Thiele, M. Bowen, S. A. Majetich, M. Kläui, I. L. Prejbeanu, B. Dieny, N. M. Dempsey, and B. Hillebrands, "The 2014 magnetism roadmap,” J. Phys. D: Appl. Phys. 47, 333001 (2014).

${ }^{14}$ A. Wu, Y. Kubota, T. Klemmer, T. Rausch, C. Peng, Y. Peng, D. Karns, X. Zhu, Y. Ding, E. Chang, Y. Zhao, H. Zhou, K. Gao, J.-U. Thiele, M. Seigler, G. Ju, and E. Gage, "HAMR areal density demonstration of $1+$ Tbpsi on spinstand,” IEEE Trans. Magn. 49, 779-782 (2013).

${ }^{15}$ N. Zhou, X. Xu, A. T. Hammack, B. C. Stipe, K. Gao, W. Scholz, and E. C. Gage, "Plasmonic near-field transducer for heat-assisted magnetic recording," Nanophotonics 3(3), 141-155 (2014).

${ }^{16} \mathrm{~B}$. V. Budaev and D. B. Bogy, "On the lifetime of plasmonic transducers in heat assisted magnetic recording," J. Appl. Phys. 112, 034512 (2012).

${ }^{17}$ B. X. Xu, Z. J. Liu, R. Ji, Y. T. Toh, J. F. Hu, J. M. Li, J. Zhang, K. D. Ye, and C. W. Chia, "Thermal issues and their effects on heat-assisted magnetic recording system (invited)," J. Appl. Phys. 111, 07 B701 (2012).

${ }^{18}$ D. Weller, G. Parker, O. Mosendz, E. Champion, B. Stipe, X. Wang, T. Klemmer, G. Ju, and A. Ajan, "A HAMR media technology roadmap to an areal density of 4 Tb/in,” IEEE Trans. Magn. 50, 1-8 (2014).

${ }^{19}$ H. J. Richter, "The transition from longitudinal to perpendicular recording," J. Phys. D: Appl. Phys. 40, R149-R177 (2007).

${ }^{20}$ S. Khizroev and D. Litvinov, "Perpendicular magnetic recording: Writing process," J. Appl. Phys. 95(9), 4521 (2004).

${ }^{21}$ M. Takahashi and S. Saito, "Advanced granular-type perpendicular recording media,” J. Magn. Magn. Mater. 320, 2868 (2008).

${ }^{22}$ R. Wood, "Future hard disk drive systems," J. Magn. Magn. Mater. 321, 555 (2009).

${ }^{23}$ M. H. Kryder and R. W. Gustafson, "High-density perpendicular recording - advances, issues, and extensibility," J. Magn. Mag. Mater. 287, 449-458 (2005).

${ }^{24}$ K. Z. Gao, O. Heinonen, and Y. Chen, "Read and write processes, and head technology for perpendicular recording," J. Magn. Magn. Mater. 321, 495-507 (2009).

${ }^{25}$ K. K. Teo, M. R. Elidrissi, K. S. Chan, and Y. Kanai, "Analysis and design of shingled magnetic recording systems," J. Appl. Phys. 111, 07B716 (2012)

${ }^{26}$ R. Wood, M. Williams, A. Kavcic, and J. Miles, "The feasibility of magnetic recording at 10 terabits per square inch on conventional media," IEEE Trans. Magn. 45, 917-923 (2009).

${ }^{27}$ W. D. Zhou, B. Liu, S. K. Yu, and W. Hua, "Inert gas filled head-disk interface for future extremely high density magnetic recording," Tribol. Lett. 33(3), 179 (2009).

${ }^{28}$ R. Wood, Y. Hsu, and M. Schultz, "Perpendicular magnetic recording technology," Technical Report, White Paper, Hitachi Storage Technology, 2007; https:/www.hgst.com/sites/default/files/resources/ PMR_white_paper_final.pdf.
${ }^{29}$ T. F. Ambrose, "Magnetic write head with thermoelectric cooling device," U.S. patent application US8031435 B2, Seagate Technology LLC (2006).

${ }^{30}$ M. A. Bashir, T. Schrefl, J. Dean, A. Goncharov, G. Hrkac, D. A. Allwood, and D. Suess, "Head and bit patterned media optimization at areal densities of $2.5 \mathrm{Tbit} / \mathrm{in}^{2}$ and beyond," J. Magn. Magn. Mater. 324(3), 269 (2012).

${ }^{31}$ Global Earth Physics - A Handbook of Physical Constants. Fundamental Physical Constants and Conversion Factors, edited by T. J. Ahrens and B. M. Moskowitz (American Geophysical Union, Washington DC, USA, 2013); available at http://spintronics.inha.ac.kr/unit.pdf.

${ }^{32}$ J. M. D. Coey, K. O’Donnell, Q. Qinian, E. Touchais, and K. H. Jack, "The magnetisation of $\alpha^{\prime \prime} \mathrm{Fe}_{16} \mathrm{~N}_{2}$," J. Phys.: Condens. Matter 6, L23-L28 (1994).

${ }^{33}$ B. D. Cullity and C. D. Graham, Introduction to Magnetic Materials, 2nd ed. (John Wiley \& Sons, Inc., Hoboken, New Jersey, 2009).

${ }^{34}$ C. M. Hurd, "Varieties of magnetic order in solids," Contemp. Phys. 23(5), 469 (1982).

${ }^{35}$ J. M. D. Coey, Magnetism and Magnetic Materials (Cambridge University Press, Cambridge, UK, 2012).

${ }^{36} \mathrm{~S}$. Blundell, Magnetism in Condensed Matter, 1st ed. (Oxford University Press, 2001).

${ }^{37}$ M. Getzlaff, Fundamentals of Magnetism (Springer, Berlin, New York, 2007).

${ }^{38}$ P. Hohenberg and W. Kohn, "Inhomogeneous electron gas," Phys. Rev. 136, B864-B871 (1964).

${ }^{39}$ W. Kohn and L. J. Sham, "Self-consistent equations including exchange and correlation effects," Phys. Rev. 140, A1133-A1138 (1965).

${ }^{40}$ U. V. Barth and L. Hedin, "A local exchange-correlation potential for the spin polarized case. I,” J. Phys. C: Solid State Phys. 5, 1629 (1972).

${ }^{41}$ J. P. Perdew, K. Burke, and Y. Wang, "Generalized gradient approximation for the exchange-correlation hole of a many-electron system," Phys. Rev. B 54, 16533-16539 (1996).

${ }^{42}$ Y. Ishikawa and S. Chikazumi, "Design of high power electromagnets," Jpn. J. Appl. Phys., Part 1 1, 155 (1962).

${ }^{43}$ S. Batra, M. Covington, T. M. Crawford, B. Crue, P. A. A. Van der Heijden, J. Jayashankar, E. C. Johns, M. Kryder, K. Minor, R. Rottmayer, U. Tran, and J. West, "A perpendicular write head design for high-density recording,” IEEE Trans. Magn. 38(1), 157 (2002).

${ }^{44}$ Y. Chen, X. Dang, Y. Liu, H. Jiang, K. Stoev, F. Liu, P. Luo, J. Wang, J. Chen, S. Gu, M. Lederman, M. Krounbi, and M. Re, "Inductive write heads using high-moment pole materials for ultrahigh-density demonstrations," IEEE Trans. Magn. 39(5), 2368-2370 (2003).

${ }^{45}$ K. Takano, Y. Liu, K. Liu, D. Z. Bai, T. Min, Y. Wu, and M. Dovek, "Micromagnetics of side shielded perpendicular magnetic recording heads," Phys. Procedia 16, 28 (2011).

${ }^{46}$ K. Hirata, Y. Ishida, T. Akashi, D. Shindo, and A. Tonomura, "Electron holography study of magnetization behavior in the writer pole of a perpendicular magnetic recording head by a $1 \mathrm{mV}$ transmission electron microscope," J. Electron Microsc. 61(5), 305 (2012).

${ }^{47}$ D. Weller, A. Moser, L. Folks, M. Best, W. Lee, M. Toney, and M. Schwickert, "High Ku materials approach to $100 \mathrm{Gbits} / \mathrm{in}^{2}$," IEEE Trans. Magn. 36(1), 10-15 (2000).

${ }^{48}$ S. Wang, D. Wei, and K.-Z. Gao, "Initial permeability and dynamic response of FeCo write pole,” IEEE Trans. Magn. 46, 1951-1954 (2010).

${ }^{49}$ R. Chantrell, J. Hannay, M. Wongsam, and A. Lyberatos, "High speed switching in magnetic recording media," IEEE Trans. Magn. 34, 349-354 (1998).

${ }^{50}$ H. Yin, J. Xue, V. R. Inturi, M. T. Kief, and M. C. Kautzky, "Write pole for recording head," U.S. patent: US20140169146 A1 (filed 2012, issued 2014), Seagate Technology LLC.

${ }^{51}$ N. Kanani, Electroplating: Basic Principles, Processes and Practice (Elsevier Ltd., Oxford, UK, 2004).

${ }^{52} \mathrm{~W}$. Sha, X. Wu, and K. G. Keong, Electroless Copper and NickelPhosphorus Plating: Processing, Characterisation and Modelling (Woodhead Publishing Ltd., Cambridge, UK, 2011).

${ }^{53}$ H. O. Pierson, Handbook of Chemical Vapor Deposition: Principles, Technology and Applications, 2nd ed. (William Andrew, Norwich, NY, 2000).

${ }^{54}$ D. M. Mattox, Handbook of Physical Vapor Deposition (PVD) Processing, 2nd ed. (Elsevier Ltd., Oxford, 2012).

${ }^{55}$ L. B. Hunt, "The early history of gold plating," Gold Bull. 6(1), 16 (1973).

${ }^{56}$ S. R. Brankovic, X. Yang, T. J. Klemmer, and M. Seigler, "Pulse electrodeposition of $2.4 \mathrm{~T} \mathrm{Co}_{37} \mathrm{Fe}_{63}$ alloys at nanoscale for magnetic recording application," IEEE Trans. Magn. 42, 132-139 (2006). 
${ }^{57}$ T. Watanabe, Nano-Plating, 1st ed. (Elsevier Ltd., Oxford, UK, 2004).

${ }^{58}$ C. D. M. Campos, A. Flacker, A. R. Vaz, S. A. Moshkalev, and E. G. O. Nobrega, "Electroplated nickel permanent magnetic films over polymeric membranes," J. Electrochem. Soc. 158(6), D330-D334 (2011).

${ }^{59}$ L. M. Monzon, Z. Diao, and J. M. D. Coey, "Electrodeposition of gadolinium in dimethylsulfoxide, and ethyldimethylbutylammonium bis(trifluoromethylsulfonyl)imide," in 216th ECS Meeting Abstracts 2009 (2009), Vol. MA2009-02, pp. 3096-3096; available at http://ma.ecsdl. org/content/MA2009-02/41/3096.

${ }^{60}$ L. M. A. Monzon, "Electrochemical characterisation of bis $(1,10-$ phenanthroline) gadolinium(III) trichloride formed in situ," J. Electroanal. Chem. 648, 47-53 (2010)

${ }^{61}$ L. M. Glukhov, A. A. Greish, and L. M. Kustov, "Electrodeposition of rare earth metals Y, Gd, Yb in ionic liquids," Russ. J. Phys. Chem. A 84, 104-108 (2010).

${ }^{62}$ X. Liu, G. Zangari, and M. Shamsuzzoha, "Structural and magnetic characterization of electrodeposited, high moment FeCoNi films," J. Electrochem. Soc. 150, C159-C168 (2003).

${ }^{63}$ H. O. Ali and I. R. A. Christie, "A review of electroless gold deposition processes,” Gold Bull. 17(4), 118 (1984).

${ }^{64}$ G. Scheunert, V. Hoffmann, R. Kullock, J. R. Whyte, R. Kirchner, S. Grafstroem, W.-J. Fischer, and L. M. Eng, "Utilizing dog-boning to build up high-aspect-ratio nanofences," J. Electrochem. Soc. 161, D26-D30 (2014).

${ }^{65}$ C. J. Brinker and A. J. Hurd, "Fundamentals of sol-gel dip-coating," J. Phys. III 4(7), 12 (1994).

${ }^{66}$ E. Hugonnot, M.-H. Delville, and J.-P. Delville, "Universal behavior of photochemical deposition in liquid solutions driven by a one-photon transition," Phys. Rev. E 75, 061602 (2007).

${ }^{67}$ B. Marchon, T. Pitchford, Y.-T. Hsia, and S. Gangopadhyay, "The headdisk interface roadmap to an areal density of $4 \mathrm{Tbit} / \mathrm{in}^{2}$," Adv. Tribol. 2013, 521086.

${ }^{68}$ B. S. Lim, A. Rahtu, and R. G. Gordon, "Atomic layer deposition of transition metals," Nat. Mater. 2, 749-754 (2003).

${ }^{69}$ T. Aaltonen, M. Ritala, Y.-L. Tung, Y. Chi, K. Arstila, K. Meinander, and M. Leskelä, “Atomic layer deposition of noble metals: Exploration of the low limit of the deposition temperature," J. Mater. Res. 19(11), 3353-3358 (2004).

${ }^{70}$ P. J. Kelly and R. D. Arnell, "Magnetron sputtering: A review of recent developments and applications," Vacuum 56, 159-172 (2000).

${ }^{71}$ J. G. Han, "Recent progress in thin film processing by magnetron sputtering with plasma diagnostics," J. Phys. D: Appl. Phys. 42, 043001 (2009).

${ }^{72}$ M. Vopsaroiu, M. Georgieva, P. J. Grundy, G. V. Fernandez, S. Manzoor, M. J. Thwaites, and K. O'Grady, "Preparation of high moment CoFe films with controlled grain size and coercivity," J. Appl. Phys. 97, 10N303 (2005).

${ }^{73}$ J. S. Colligon, "Ion-assisted sputter deposition," Philos. Trans. R. Soc. Lond. A 362, 103 (2004).

${ }^{74}$ Z. Lu, Y. Fukuma, W. H. Butler, H. Fujiwara, G. J. Mankey, and S. Matsunuma, "Magnetic anisotropy of FeCo films induced by obliquely sputtered Ru underlayers," IEEE Trans. Magn. 45(10), 4008 (2009).

${ }^{75}$ H. B. Nie, S. Y. Xu, C. K. Ong, Q. Zhan, D. X. Li, and J. P. Wang, "Inplane magnetic anisotropy in RF sputtered Fe-N thin films," Thin Solid Films 440, 35-40 (2003).

${ }^{76}$ Handbook of Thin Film Deposition Techniques: Principles, Methods, Equipment and Applications, edited by K. Seshan, 2nd ed. (CRC Press, Norwich, NY, 2002).

${ }^{77}$ J. E. Greene, "Tracing the 5000-year recorded history of inorganic thin films from -3000 BC to the early 1900s AD,” Appl. Phys. Rev. 1, 041302 (2014).

${ }^{78} \mathrm{~K}$. Dumesnil and S. Andrieu, "Epitaxial magnetic layers grown by MBE: model systems to study the physics in nanomagnetism and spintronic," in "Molecular Beam Epitaxy" (Elsevier, Oxford, 2013), Chap. 20.

${ }^{79}$ Y. Sugita, K. Mitsuoka, M. Komuro, H. Hoshiya, Y. Kozono, and M. Hanazono, "Giant magnetic moment and other magnetic properties of epitaxially grown $\mathrm{Fe}_{16} \mathrm{~N}_{2}$ single-crystal films (invited)," J. Appl. Phys. 70, 5977-5982 (1991).

${ }^{80} \mathrm{~W}$. F. Smith, Foundations of Materials Science and Engineering - Crystal Structures and Crystal Geometry (McGraw-Hill Higher Education, University of Central Florida, USA, 2004).

${ }^{81}$ D. O. Smith, "Development of a vibrating-coil magnetometer," Rev. Sci. Instrum. 27, 261-268 (1956).

${ }^{82}$ R. L. Fagaly, "Superconducting quantum interference device instruments and applications," Rev. Sci. Instrum. 77, 101101 (2006).
${ }^{83}$ M. Sawicki, W. Stefanowicz, and A. Ney, "Sensitive SQUID magnetometry for studying nanomagnetism," Semicond. Sci. Technol. 26, 064006 (2011).

${ }^{84}$ J. Stoehr, H. A. Padmore, S. Anders, T. Stammler, and M. R. Scheinfein, "Principles of x-ray magnetic dichroism spectromicroscopy," Surf. Rev. Lett. 05, 1297-1308 (1998).

${ }^{85}$ M. Farle, "Ferromagnetic resonance of ultrathin metallic layers," Rep. Prog. Phys. 61, 755 (1998).

${ }^{86}$ C. Wang, H. Seinige, and M. Tsoi, "Ferromagnetic resonance driven by an ac current: A brief review," Low Temp. Phys. 39, 247-251 (2013).

${ }^{87}$ J. Chang-Jun, F. Xiao-Long, and X. De-Sheng, "High frequency magnetic properties of ferromagnetic thin films and magnetization dynamics of coherent precession," Chin. Phys. B 24, 057504 (2015).

${ }^{88}$ N. A. Buznikov, I. T. Iakubov, A. L. Rakhmanov, and A. O. Sboychakov, "High-frequency magnetic permeability of nanocomposite film," J. Magn. Magn. Mater. 293, 938-946 (2005).

${ }^{89}$ D. Weller, O. Mosendz, H. J. Richter, G. Parker, S. Pisana, T. S. Santos, J. Reiner, O. Hellwig, B. Stipe, and B. Terris, "Sub-nanosecond heat assisted magnetic recording of FePt media," in Ultrafast Magnetism I, Springer Proceedings in Physics, edited by J.-Y. Bigot, W. Hübner, T. Rasing, and R. Chantrell (Springer International Publishing, 2015), Vol. 159, pp. 228-231.

${ }^{90}$ J.-Y. Juang and D. B. Bogy, "Air-bearing effects on actuated thermal pole-tip protrusion for hard disk drives," J. Tribol. 129(3), 570 (2007).

${ }^{91}$ J. Fritzsche, H. Li, H. Zheng, K. Amemiya, and F. E. Talke, "The effect of air bearing contour design on thermal pole-tip protrusion," Microsyst. Technol. 17, 813 (2011).

${ }^{92}$ T. Rausch, J. D. Trantham, J. W. Dykes, H. Dakroub, C. P. Henry, E. C. Gage, R. H. Andruet, J. G. Wessel, J. D. Kiely, B. D. Buch, and B. Vikramaditya, "Heat assisted magnetic recording device with pre-heated write element," U.S. patent: US9019646 B2 (filed 2013, issued 2015), Seagate LLC.

${ }^{93}$ S. Y. Dan'kov, A. M. Tishin, V. K. Pecharsky, and K. A. Gschneidner, "Magnetic phase transitions and the magnetothermal properties of gadolinium,” Phys. Rev. B 57, 3478 (1998).

${ }^{94}$ X. Moya, L. E. Hueso, F. Maccherozzi, A. I. Tovstolytkin, D. I. Podyalovskii, C. Ducati, L. C. Phillips, M. Ghidini, O. Hovorka, A. Berger, M. E. Vickers, E. Defay, S. S. Dhesi, and N. D. Mathur, "Giant and reversible extrinsic magnetocaloric effects in $\mathrm{La}_{0.7} \mathrm{Ca}_{0.3} \mathrm{MnO}_{3}$ films due to strain," Nat. Mater. 12, 52 (2012).

${ }^{95}$ K. A. Gschneidner, Jr., V. K. Pecharsky, and A. O. Tsokol, "Recent developments in magnetocaloric materials," Rep. Prog. Phys. 68, 1479 (2005).

${ }^{96}$ S. P. Mathew and S. N. Kaul, "Tuning magnetocaloric effect with nanocrystallite size," Appl. Phys. Lett. 98(17), 172505 (2011).

${ }^{97}$ B. G. Shen, J. R. Sun, F. X. Hu, H. W. Zhang, and Z. H. Cheng, "Recent progress in exploring magnetocaloric materials," Adv. Mater. 21, 4545 (2009).

${ }^{98}$ O. Gutfleisch, M. A. Willard, E. Brueck, C. H. Chen, S. G. Sankar, and J. P. Liu, "Magnetic materials and devices for the 21st century: Stronger, lighter, and more energy efficient," Adv. Mater. 23, 821 (2011).

${ }^{99}$ K. Gschneidner, Jr. and V. Pecharsky, "Thirty years of near room temperature magnetic cooling: Where we are today and future prospects," Int. J. Refrig. 31, 945-961 (2008).

${ }^{100}$ J. R. Hull and K. L. Uherka, "Magnetic heat pumps for near-room-temperature applications," Energy 14(4), 177 (1989).

${ }^{101}$ E. Brueck, O. Tegus, D. T. C. Thanh, and K. H. J. Buschow, "Magnetocaloric refrigeration near room temperature (invited)," J. Magn. Magn. Mater. 310, 2793 (2007).

${ }^{102}$ Y. Zhang, P. J. Lampen, T.-L. Phan, S.-C. Yu, H. Srikanth, and M.-H. Phan, "Tunable magnetocaloric effect near room temperature in $\mathrm{La}_{0.7-\mathrm{x}}$ $\mathrm{Pr}_{\mathrm{x}} \mathrm{Sr}_{0.3} \mathrm{MnO}_{3}(0.02 \leq x \leq 0.30)$ manganites," J. Appl. Phys. 111, 063918 (2012).

${ }^{103}$ A. Fukushima, H. Kubota, A. Yamamoto, Y. Suzuki, and S. Yuasa, "Peltier effect in metallic junctions with CPP structure," IEEE Trans. Magn. 41(10), 2571 (2005).

${ }^{104}$ A. Sugihara, M. Kodzuka, K. Yakushiji, H. Kubota, S. Yuasa, A. Yamamoto, K. Ando, K. Takanashi, T. Ohkubo, K. Hono, and A. Fukushima, "Giant Peltier effect in a submicron-sized $\mathrm{Cu}$ _ni/Au junction with nanometer-scale phase separation," Appl. Phys. Express 3, 065204 (2010).

${ }^{105}$ G. E. W. Bauer, A. H. MacDonald, and S. Maekawa, "Spin caloritronics," Solid State Commun. 150(11), 459 (2010).

${ }^{106}$ G. E. W. Bauer, E. Saitoh, and B. J. Van Wees, "Spin caloritronics," Nat. Materi. 11, 391 (2012). 
${ }^{107}$ A. Miner, "The compatibility of thin films and nanostructures in thermoelectric cooling systems," J. Heat Transfer 129, 805-812 (2006).

${ }^{108}$ Y. Hishinuma, T. H. Geballe, B. Y. Moyzhes, and T. W. Kenny, "Measurements of cooling by room-temperature thermionic emission across a nanometer gap," J. Appl. Phys. 94(7), 4690 (2003).

${ }^{109}$ K. W. Ng, S. H. Leong, Z. Yuan, B. Liu, and Y. Ma, "Heat assisted magnetic recording by combined field emission and moderate ionization in air," Appl. Phys. Lett. 91, 172511 (2007).

${ }^{110}$ L. Zhang, X. T. Zu, J. G. Peng, Z. Yan, and Z. Y. Zhong, "Thermo-magnetic recording on a $\mathrm{Co} / \mathrm{Pt}$ perpendicular film by a sharp metallic probe," Nanotechnology 19(8), 085714 (2008).

${ }^{111} \mathrm{C}$. O'Briain Fallon, "Thermal modelling of a magnetic recording write pole cooled by electron emission," Master of Engineering, Electronic Systems, Dublin City University, Dublin, Ireland, 2012.

${ }^{112}$ X. L. Ruan and M. Kaviany, "Advances in laser cooling of solids," J. Heat Transfer 129, 3-10 (2006).

${ }^{113}$ R. Epstein and M. Sheik-Bahae, "Optical refrigeration in solids: Fundamentals and overview," in Optical Refrigeration: Science and Applications of Laser Cooling of Solids (Wiley, 2009), Vol. 1, p. 258.

${ }^{114}$ G. Nemova and R. Kashyap, "Laser cooling of solids," Rep. Prog. Phys. 73, 086501 (2010).

${ }^{115}$ M. Sheik-Bahae, S. Melgaard, D. Seletskiy, A. Albrecht, M. Ghasemkhani, and R. Epstein, "Laser cooling in solids: Demonstration of $115 \mathrm{~K}$ all-solid-state cryocooler," in 2013 IEEE Photonics Conference (IPC) (2013), pp. 141-142.

${ }^{116}$ D. V. Seletskiy, S. D. Melgaard, S. Bigotta, A. Di Lieto, M. Tonelli, and M. Sheik-Bahae, "Laser cooling of solids to cryogenic temperatures," Nat. Photonics 4, 161-164 (2010).

${ }^{117}$ G. V. Brown, "Magnetic heat pumping near room temperature," J. Appl. Phys. 47, 3673 (1976).

${ }^{118}$ B. Yu, M. Liu, P. W. Egolf, and A. Kitanovski, "A review of magnetic refrigerator and heat pump prototypes built before the year 2010," Int. J. Refrig. 33, 1029 (2010).

${ }^{119} \mathrm{~A}$. Rowe and A. Tura, "Experimental investigation of a three-material layered active magnetic regenerator,” Int. J. Refrig. 29, 1286 (2006).

${ }^{120}$ L.-X. Chang, G. Xiong, L. Wang, P. Cheng, and B. Zhao, "A 24-Gd nano-capsule with large magnetocaloric effect," Chem. Commun. 49(11), 1055 (2013).

${ }^{121}$ H. H. Busta, "Vacuum microelectronics-I," J. Micromech. 2, 43 (1992).

${ }^{122}$ A. Shakouri, "Nanoscale thermal transport and microrefrigerators on a chip," Proc. IEEE 94(8), 1613 (2006).

${ }^{123}$ B. Guha, C. Otey, C. B. Poitras, S. Fan, and M. Lipson, "Near-field radiative cooling of nanostructures," Nano Lett. 12, 4546-4550 (2012).

${ }^{124}$ J. Zhang, D. Li, R. Chen, and Q. Xiong, "Laser cooling of a semiconductor by 40 kelvin," Nature 493, 504-508 (2013).

${ }^{125}$ G. Nemova and R. Kashyap, "Recent advances in laser cooling of solids," Proc. SPIE 8915, 89151T (2013).

${ }^{126} \mathrm{P}$. Weiss, "The magnetic properties of the alloys of the ferromagnetic metals: Iron-nickel, nickel-cobalt, cobalt-iron," Trans. Faraday Soc. 8, 149-156 (1912).

${ }^{127} \mathrm{G}$. W. Elmen, "Magnetic material and appliance," U.S. patent: US1739752 A (filed 1927, issued 1929), Bell Telephone Laboratory Inc.

${ }^{128}$ T. Sourmail, "Near equiatomic FeCo alloys: Constitution, mechanical and magnetic properties," Prog. Mater. Sci. 50, 816 (2005).

${ }^{129}$ Y. Chen, K. Sin, H. Jiang, Y. Tang, K. Sasaki, A. Torabi, L. Wang, M. Park, D. Bai, Y. Shen, P. Luo, F. Liu, K. Stoev, W. Lin, and J. Zhu, "High moment materials and fabrication processes for shielded perpendicular write head beyond $200 \mathrm{~Gb} / \mathrm{in}^{2}$," IEEE Trans. Magn. 43(2), 609 (2007).

${ }^{130}$ M. T. Kief, V. Inturi, M. Benakli, I. Tabakovic, M. Sun, O. Heinonen, S. Riemer, and V. Vas'ko, "High magnetic saturation poles for advanced perpendicular writers," IEEE Trans. Magn. 44, 113-118 (2008).

${ }^{131}$ H. S. Jung, W. D. Doyle, and S. Matsunuma, "Influence of underlayers on the soft properties of high magnetization FeCo films," J. Appl. Phys. 93(10), 6462 (2003).

${ }^{132}$ V. Inturi, H. Yin, M. Kief, M. Hadley, and C. Mathieu, "Practical FeCo films for perpendicular writer pole," IEEE Trans. Magn. 48(5), 1718 (2012).

${ }^{133}$ C. Kuhrt and L. Schultz, "Formation and magnetic properties of nanocrystalline mechanically alloyed Fe-Co and Fe-Ni," J. Appl. Phys. 73, 6588-6590 (1993).

${ }^{134}$ C. Binns, K. N. Trohidou, J. Bansmann, S. H. Baker, J. A. Blackman, J.P. Bucher, D. Kechrakos, A. Kleibert, S. Louch, K.-H. Meiwes-Broer, G. M. Pastor, A. Perez, and Y. Xie, "The behaviour of nanostructured magnetic materials produced by depositing gas-phase nanoparticles," J. Phys. D: Appl. Phys. 38, R357 (2005).

${ }^{135}$ A. P. Malozemoff, A. R. Williams, and V. L. Moruzzi, "Band-gap theory of strong ferromagnetism: Application to concentrated crystalline and amorphous Fe- and Co-metalloid alloys," Phys. Rev. B 29, 1620-1632 (1984).

${ }^{136}$ D. I. Bardos, "Mean magnetic moments in BCC Fe-Co alloys," J. Appl. Phys. 40, 1371-1372 (1969).

${ }^{137}$ J. G. Booth, "Ferromagnetic transition metal intermetallic compounds," in Handbook of Ferromagnetic Materials Booth, edited by E. P. Wohlfarth and K. H. J. Buschow (Elsevier, North-Holland, Amsterdam, Netherlands, 1998), Vol. 4, p. 211.

${ }^{138}$ R. M. Bozorth, Ferromagnetism (Van Nostrand, Princeton, NJ, USA, 1951).

${ }^{139}$ G. Parette and I. Mirebeau, "Magnetic moment distribution in iron-cobalt alloys," Phys. B 156-157, 721-723 (1989).

${ }^{140}$ O. Eriksson, A. Bergman, B. Skubic, L. Nordstroem, and E. Holmstroem, "First-principle modeling of magnetic moments in FeCo multilayers" (unpublished).

${ }^{141}$ J. M. MacLaren, T. C. Schulthess, W. H. Butler, R. Sutton, and M. McHenry, "Electronic structure, exchange interactions, and Curie temperature of FeCo," J. Appl. Phys. 85(8), 4833 (1999).

${ }^{142}$ D. W. Clegg and R. A. Buckley, "The disorder - order transformation in iron-cobalt-based alloys," Met. Sci. J. 7, 48 (1973).

${ }^{143}$ S. X. Wang, N. X. Sun, M. Yamaguchi, and S. Yabukami, "Sandwich films: Properties of a new soft magnetic material," Nature 407, 150-151 (2000).

${ }^{144}$ J. Horkans, D. J. Seagle, and I. C. H. Chang, "Electroplated magnetic media with vertical anisotropy," J. Electrochem. Soc. 137(7), 2056-2061 (1990).

${ }^{145}$ H. J. Cho, S. Bhansali, and C. H. Ahn, "Electroplated thick permanent magnet arrays with controlled direction of magnetization for MEMS application,” J. Appl. Phys. 87, 6340-6342 (2000).

${ }^{146}$ C. Campos, A. Flacker, S. Moshkalev, and E. Nobrega, "Comparative analysis of thin Ni and CoNiMnP magnetic films," Thin Solid Films 520, 4871-4874 (2012).

${ }^{147}$ S. Guan and B. J. Nelson, "Electrodeposition of low residual stress CoNiMnP hard magnetic thin films for magnetic MEMS actuators," J. Magn. Magn. Mater. 292, 49-58 (2005).

${ }^{148}$ Y. Okada, H. Hoshiya, T. Okada, and M. Fuyama, "Magnetic properties of FeCo multilayered films for single pole heads," IEEE Trans. Magn. 40, 2368-2370 (2004).

${ }^{149}$ T. Shimatsu, H. Katada, I. Watanabe, H. Muraoka, and Y. Nakamura, "Effect of lattice strain on soft magnetic properties in $\mathrm{FeCo} / \mathrm{NiFe}(\mathrm{Cr})$ thin films with 2.4 T," IEEE Trans. Magn. 39, 2365-2367 (2003).

${ }^{150}$ X. Liu, H. Kanda, and A. Morisako, "The effect of underlayers on FeCo thin films,” J. Phys.: Conf. Ser. 266, 012037 (2011).

${ }^{151}$ T. Kawai, Y. Asai, M. Ohtake, S. Takeda, and M. Futamoto, "Dynamic magnetic properties of $\mathrm{Fe}_{70} \mathrm{Co}_{30}(100)$ single-crystal thin films deposited at various substrate temperatures," EPJ Web Conf. 40, 13001 (2013).

${ }^{152}$ B. Bhoi, V. Singh, A. Perumal, and V. Srinivas, "Influence of synthesis processes on microstructure and magnetic properties in $\mathrm{Fe}_{70} \mathrm{Co}_{30}$ alloy nanoparticles," AIP Conf. Proc. 1347, 115 (2011).

${ }^{153}$ A. L. Elias, J. A. Rodriguez-Manzo, M. R. McCartney, D. Golberg, A. Zamudio, S. E. Baltazar, F. Lopez-Urias, E. Munoz-Sandoval, L. Gu, C. C. Tang, D. J. Smith, Y. Bando, H. Terrones, and M. Terrones, "Production and characterization of single-crystal FeCo nanowires inside carbon nanotubes," Nano Lett. 5(3), 467 (2005).

${ }^{154}$ I. Shao, M. W. Chen, R. C. Cammarata, P. C. Searson, and S. M. Prokes, "Deposition and characterization of $\mathrm{Fe}_{0.55} \mathrm{Co}_{0.45}$ nanowires," J. Electrochem. Soc. 154(11), D572 (2007).

${ }^{155}$ T. Nishiyama, M. Ohtake, F. Kirino, and M. Futamoto, "Preparation and structural characterization of FeCo epitaxial thin films on insulating single-crystal substrates,” J. Appl. Phys. 107(9), 09A306 (2010).

${ }^{156}$ M. E. McHenry, M. A. Willard, and D. E. Laughlin, "Amorphous and nanocrystalline materials for applications as soft magnets," Prog. Mater. Sci. 44, 291-433 (1999).

${ }^{157}$ I. Skorvanek, J. Marcin, J. Turcanova, J. Kovac, and P. Svec, "FeCobased soft magnetic nanocrystalline alloys," Acta Electrotech. Inf. 10(3), 14 (2010).

${ }^{158}$ J. Marcin, M. Capik, J. Kovac, P. Svec, I. Petryshynets, F. Kovac, and I. Skorvanek, "Tuning of magnetic properties and domain structure in FeCo- and FeSi-based soft magnetic alloys by thermal processing under magnetic field," Acta Electrotech. Inf. 13(1), 91 (2013). 
${ }^{159}$ N. X. Sun and S. X. Wang, "Soft high saturation magnetization $\left(\mathrm{Fe}_{0.7} \mathrm{Co}_{0.3}\right)_{1-\mathrm{x}} \mathrm{N}_{\mathrm{x}}$ thin films for inductive write heads," IEEE Trans. Magn. 36(5), 2506 (2000).

${ }^{160}$ K. Shintaku, K. Ise, T. Kiya, K. Yamakawa, and K. Ouchi, "Fe-Co-Al-O soft magnetic films for main pole of CF-SPT head and underlayer of perpendicular double-layered media," J. Magn. Magn. Mater. 287, 265-270 (2005).

${ }^{161}$ M. K. Minor, T. M. Crawford, T. J. Klemmer, Y. Peng, and D. E. Laughlin, "Stress dependence of soft, high moment and nanocrystalline FeCoB films," J. Appl. Phys. 91(10), 8453 (2002).

${ }^{162}$ H. Jung and W. Doyle, "CoFe-IrMn exchange-coupled soft underlayers for perpendicular media," IEEE Trans. Magn. 38, 2015-2017 (2002).

${ }^{163}$ H. Jiang, K. Sin, and Y. Chen, "High moment soft FeCoN/NiFe laminated thin films," IEEE Trans. Magn. 41(10), 2896 (2005).

${ }^{164}$ B. R. Craig, S. McVitie, J. N. Chapman, A. B. Johnston, and D. O. O'Donnell, "Transmission electron microscopy study of CoFe films with high saturation magnetization,” J. Appl. Phys. 100, 053915 (2006).

${ }^{165}$ G. Scheunert, C. Ward, W. R. Hendren, A. A. Lapicki, R. Hardeman, M. Mooney, M. Gubbins, and R. M. Bowman, "Influence of strain and polycrystalline ordering on magnetic properties of high moment rare earth metals and alloys," J. Phys. D: Appl. Phys. 47, 415005 (2014).

${ }^{166}$ J. M. MacLaren, M. E. McHenry, S. Crampin, and M. E. Eberhart, "Magnetic and electronic properties of $\mathrm{Au} / \mathrm{Fe}$ superlattices and interfaces," J. Appl. Phys. 67(9), 5406 (1990).

${ }^{167}$ D. C. A. Stoeffler, "Calculations of electronic and magnetic structures in ultra-thin magnetic systems," J. Magn. Magn. Mater. 165, 62 (1997).

${ }^{168}$ H. Muehlbauer, C. Mueller, and G. Dumpich, "Stabilization of high spin FCC $\mathrm{Fe}$ in $(\mathrm{Fe} / \mathrm{Pd}) \mathrm{n}$ multilayers," J. Magn. Magn. Mater. 192, 423 (1999).

${ }^{169}$ T. Ambrose, J. J. Krebs, K. Bussmann, and G. A. Prinz, "Magnetic and structural properties of face-centered-cubic $\mathrm{Fe}_{\mathrm{x}} \mathrm{Co}_{1-\mathrm{x}}$ alloys on diamond," J. Appl. Phys. 85, 5066 (1999).

${ }^{170}$ S. Crampin, "Fe on $\mathrm{Au}(001)$ : Magnetism and band formation," J. Phys.: Condens. Matter 5, 4647 (1993).

${ }^{171}$ C. L. Wooten, J. Chen, G. A. Mulhollan, J. L. Erskine, and J. T. Markert, "Direct observation of enhanced magnetic moments in $\mathrm{Fe} / \mathrm{Ag}(100)$," Phys. Rev. B 49(14), 10023 (1994).

${ }^{172}$ S. Ohnishi, A. J. Freeman, and M. Weinert, "Surface magnetism of Fe(001),” Phys. Rev. B 28(12), 6741 (1983).

${ }^{173}$ R. Richter, J. G. Gay, and J. R. Smith, "Spin separation in a metal overlayer,” Phys. Rev. Lett. 54(25), 2704 (1985).

${ }^{174} \mathrm{~S}$. Ohnishi, M. Weinert, and A. J. Freeman, "Interface magnetism in metals: Ag/Fe(001)," Phys. Rev. B 30, 36 (1984).

${ }^{175}$ S. Bluegel, B. Drittler, R. Zeller, and D. H. Dederichs, "Magnetic properties of $3 \mathrm{~d}$ transition metal monolayers on metal substrates," Appl. Phys. A 49(6), 547 (1989).

${ }^{176}$ F. Pan, K. Tao, and X. Liu, "Enhancement of magnetic moment of iron atoms in the Fe/Au nanomultilayers," J. Appl. Phys. 74(3), 1929 (1993).

${ }^{177}$ E. Jal, M. Dabrowski, J.-M. Tonnerre, M. Przybylski, S. Grenier, N. Jaouen, and J. Kirschner, "Magnetization profile across Au-covered BCC Fe films grown on a vicinal surface of $\mathrm{Ag}(001)$ as seen by x-ray resonant magnetic reflectivity," Phys. Rev. B 87, 224418 (2013).

${ }^{178}$ D. Stoeffler, K. Ounadjela, J. Sticht, and F. Gautier, "Magnetic polarization of the Pd spacer and interlayer magnetic couplings in Fe/Pd (001) superlattices: First principles calculations," Phys. Rev. B 49(1), 299 (1994).

${ }^{179}$ L. Cheng, Z. Altounian, D. H. Ryan, J. O. Stroem-Olsen, and M. Sutton, "Pd polarization and interfacial moments in Pd-Fe multilayers," Phys. Rev. B 69, 144403 (2004).

${ }^{180}$ S. D. Bader, E. R. Moog, and P. Gruenberg, "Magnetic hysteresis of epitaxially-deposited iron in the monolayer range: A Kerr effect experiment in surface magnetism,” J. Magn. Magn. Mater. 53(4), L295 (1986).

${ }^{181}$ C. A. F. Vaz, J. A. C. Bland, and G. Lauhoff, "Magnetism in ultrathin film structures,” Rep. Prog. Phys. 71, 056501 (2008).

${ }^{182}$ F. Wilhelm, P. Poulopoulos, V. Kapaklis, J.-P. Kappler, N. Jaouen, A. Rogalev, A. N. Yaresko, and C. Politis, "Au and Fe magnetic moments in disordered Au-Fe alloys," Phys. Rev. B 77, 224414 (2008).

${ }^{183}$ B. Sanyal, P. Biswas, T. Saha-Dasgupta, A. Mookerjee, A.-U. Huda, N. Choudhury, M. Ahmed, and A. Halder, "Electrical and magnetic properties of AuFe alloys," J. Phys.: Condens. Matter 11, 1833 (1999).

${ }^{184}$ K. Fukamichi, T. Satoh, and T. Masumoto, "High magnetic moment of Fe-Au-B sputtered amorphous alloys," J. Appl. Phys. 53(11), 7741 (1982).
${ }^{185}$ A. Bhattacharjee, M. Ahmed, A. Mookerjee, and A. Halder, "Effect of alloying on the electronic structure and magnetic properties of $\mathrm{Fe}, \mathrm{Co}$ and Ni with Au and Ag," Bull. Mater. Sci. 26(1), 199 (2003).

${ }^{186}$ X. Y. Li, L. T. Kong, and B. X. Liu, "Evidence of a high-spin ferromagnetic state in fcc-structured Fe-Ag (Au) alloys synthesized by ion beam manipulation,” J. Phys.: Condens. Matter 17, 4647 (2005).

${ }^{187}$ M. Birsan, B. Fultz, and L. Anthony, "Magnetic properties of bcc Fe-Pd extended solid solutions,” Phys. Rev. B 55(17), 11502 (1997).

${ }^{188}$ S. Kauffmann-Weiss, S. Hamann, M. E. Gruner, L. Schultz, A. Ludwig, and S. Faehler, "Enhancing magnetocrystalline anisotropy of the $\mathrm{Fe}_{70} \mathrm{Pd}_{30}$ magnetic shape memory alloy by adding $\mathrm{Cu}$," Acta Mater. 60, 6920 (2012).

${ }^{189}$ H.-J. Moon, W. Kim, S.-J. Oh, J. Park, J.-G. Park, E.-J. Cho, J. I. Lee, and H.-C. Ri, "Magnetic properties and electronic structures of (Ru,Fe) and (Rh,Fe) alloys," J. Korean Phys. Soc. 36(1), 49 (2000).

${ }^{190}$ D. H. Ryan, J. O. Stroem-Olsen, W. B. Muir, J. M. Cadogan, and J. M. D. Coey, "Magnetic properties of iron-rich Fe-Sc glasses," Phys. Rev. B 40, 11208-11214 (1989).

${ }^{191}$ S. Inoue, H. Y. Y. Ko, and T. Suzuki, "Magnetic properties of singlecrystalline FeRh alloy thin films," IEEE Trans. Magn. 44(11), 2875-2878 (2008).

${ }^{192}$ J. Cao, N. T. Nam, S. Inoue, H. Y. Y. Ko, N. N. Phuoc, and T. Suzuki, "Magnetization behaviors for FeRh single crystal thin films," J. Appl. Phys. 103(7), 07F501 (2008).

${ }^{193}$ S. Yuasa, H. Miyajima, Y. Otani, and A. Sakuma, "Magnetism of bodycentered tetragonal $\mathrm{FeRh}_{1-\mathrm{x}} \mathrm{Pd}_{\mathrm{x}}$ alloys (I) magnetic properties," J. Phys. Soc. Jpn. 64, 4906-4913 (1995).

${ }^{194}$ J. van Driel, R. Coehoorn, G. J. Strijkers, E. Bruck, and F. R. de Boer, "Compositional dependence of the giant magnetoresistance in $\mathrm{Fe}_{\mathrm{x}} \mathrm{Rh}_{1-\mathrm{x}}$ thin films," J. Appl. Phys. 85(2), 1026 (1999).

${ }^{195}$ J.-U. Thiele, S. Maat, and E. E. Fullerton, "FeRh/FePt exchange spring films for thermally assisted magnetic recording media," Appl. Phys. Lett. 82(17), 2859-2861 (2003).

${ }^{196}$ J.-U. Thiele, S. Maat, E. E. Fullerton, and J. L. Robertson, "Magnetic and structural properties of FePt-FeRh exchange spring films for thermally assisted magnetic recording media," IEEE Trans. Magn. 40, 2537 (2004).

${ }^{197}$ K. Noma, M. Matsuoka, H. Kanai, Y. Uehara, K. Nomura, and N. Awaji, "Enhancement of saturation magnetization in sputtered FeCoPd alloy and $[\mathrm{FeCo}-\mathrm{Pd}]_{\mathrm{n}}$ super-lattice films at room temperature," IEEE Trans. Magn. 41(10), 2920 (2005).

${ }^{198}$ K. Noma, H. Kanai, Y. Uehara, T. Nakamura, H. Osawa, S. Doi, K. Nomura, and N. Awaji, "X-ray magnetic circular dichroism studies of the enhancement of $\mathrm{Fe}$ spin moment in $\left(\mathrm{Fe}_{70} \mathrm{Co}_{30} \mathrm{Pd}\right)_{\mathrm{n}}$ superlattice films with high saturation magnetization," J. Appl. Phys. 101, 09D111 (2007).

${ }^{199}$ M. J. Walock, H. Ambaye, M. Chshiev, F. R. Klose, W. H. Butler, and G. J. Mankey, "High magnetization FeCo/Pd multilayers," J. Vac. Sci. Technol., A 26(4), 731 (2008).

${ }^{200}$ D. C. A. Stoeffler, K. Onuadjela, J. Sticht, and F. Gautier, "Ab initio study of the interlayer magnetic couplings in Fe/Pd(001) superlattices and of the polarization induced in the Fe and Pd layers," J. Appl. Phys. 75(10), 6467 (1994).

${ }^{201}$ L. Szunyogh, B. Ujfalussy, U. Pustogowa, and P. Weinberger, "Overlayer-dependent magnetic moment and anisotropy of a Co monolayer on Cu(100),” Phys. Rev. B 57(15), 8838 (1998).

${ }^{202}$ A. Walczak, T. Slusarski, A. Lehmann-Szweykowska, and G. Kamieniarz, "Structural and magnetic properties of Co thin films on Au(111) substrates," Acta Phys. Pol., A 121(3), 653 (2012); available at http://przyrbwn.icm.edu.pl/APP/PDF/121/a121z3p16.pdf.

${ }^{203}$ P. Srivastava, F. Wilhelm, A. Ney, M. Farle, H. Wende, N. Haack, G. Ceballos, and K. Baberschke, "Magnetic moments and Curie temperatures of Ni and Co thin films and coupled trilayers," Phys. Rev. B 58(9), 5701 (1998).

${ }^{204}$ T. Nakagawa, Y. Takagi, Y. Matsumoto, and T. Yokoyama, "Enhancements of spin and orbital magnetic moments of submonolayer $\mathrm{Co}$ on $\mathrm{Cu}(001)$ studied by x-ray magnetic circular dichroism using superconducting magnet and liquid He cryostat," Jpn. J. Appl. Phys., Part 1 47(4), 2132 (2008).

${ }^{205}$ G. Moulas, A. Lehnert, S. Rusponi, J. Zabloudil, C. Etz, S. Ouazi, M. Etzkorn, P. Bencok, P. Gambardella, P. Weinberger, and H. Brune, "High magnetic moments and anisotropies for $\mathrm{Fe}_{\mathrm{x}} \mathrm{Co}_{1-\mathrm{x}}$ monolayers on Pt(111)," Phys. Rev. B 78, 214424 (2008).

${ }^{206}$ A. Guendel, T. Devolder, C. Chappert, J. E. Schmidt, R. Cortes, and P. Allongue, "Electrodeposition of $\mathrm{Fe} / \mathrm{Au}(111)$ ultrathin layers with perpendicular magnetic anisotropy," Phys. B 354, 282 (2004). 
${ }^{207}$ Y. Xu, D. Freeland, M. Tselepi, C. Guertler, W. Lee, J. Bland, S. Holmes, N. Patel, and D. Ritchie, "Ferromagnetic/III-V semiconductor heterostructures and magneto-electronic devices," IEEE Trans. Magn. 35, 3661-3663 (1999).

${ }^{208} \mathrm{C}$. M. Teodorescu and D. Luca, "Comparative study of magnetism and interface composition in Fe/GaAs(l $\left.\begin{array}{lll}1 & 0 & 0\end{array}\right)$ and Fe/InAs(l $\left.\begin{array}{lll}1 & 0 & 0\end{array}\right)$," Surf. Sci. 600, 4200-4204 (2006).

${ }^{209}$ T. Hupfauer, A. Matos-Abiague, M. Gmitra, F. Schiller, J. Loher, D. Bougeard, C. H. Back, J. Fabian, and D. Weiss, "Emergence of spin-orbit fields in magnetotransport of quasi-two-dimensional iron on gallium arsenide," Nat. Commun. 6, 7374 (2015).

${ }^{210}$ J. A. C. Bland, S. J. Blundell, M. Gester, R. D. Bateson, J. Singleton, U. J. Cox, C. A. Lucas, W. C. K. Poon, and J. Penfold, "Magnetization and magnetoresistance of Co/GaAs(001) films," J. Magn. Magn. Mater. 115, 359-365 (1992).

${ }^{211}$ C. Scheck, P. Evans, R. Schad, G. Zangari, J. R. Williams, and T. F. Isaacs-Smith, "Structure and magnetic properties of electrodeposited $\mathrm{Ni}$ films on n-GaAs(001)," J. Phys.: Condens. Matter 14, 12329 (2002).

${ }^{212}$ S. A. Haque, A. Matsuo, Y. Seino, Y. Yamamoto, S. Yamada, and H. Hori, "Effect of GaAs substrate on the magnetic properties of Ni film," Phys. B: Condens. Matter 305, 121-126 (2001).

${ }^{213}$ S. A. Haque, A. Matsuo, Y. Yamamoto, and H. Hori, "Threshold thickness and magnetic properties of a composite Ni/GaAs (001)," Jpn. J. Appl. Phys., Part 1 41, 3678 (2002).

${ }^{214}$ A. Filipe, A. Schuhl, and P. Galtier, "Structure and magnetism of the Fe/ GaAs interface,” Appl. Phys. Lett. 70, 129-131 (1997).

${ }^{215}$ Y. Sugita, H. Takahashi, M. Takahashi, M. Komuro, I. Masukazu, R. Imura, and T. Kambe, "Magnetic and electrical properties of singlephase, single-crystal $\mathrm{Fe}_{16} \mathrm{~N}_{2}$ films epitaxially grown by molecular beam epitaxy (invited)," J. Appl. Phys. 79(8), 5576 (1996).

${ }^{216}$ M. S. Patwari and R. H. Victora, "Search for high magnetic moment recording head material: Manganese compounds," Phys. Rev. B 64, 214417 (2001).

${ }^{217}$ M. J. Besnus, A. Herr, K. Le Dang, P. Veillet, A. S. Schaafsma, I. Vincze, F. Van der Woude, and G. H. M. Calis, "Magnetic moments in manganese containing intermetallic compounds," J. Phys. F: Met. Phys. 12, 2393 (1982).

${ }^{218}$ N. I. Medvedeva, D. C. Van Aken, and J. E. Medvedeva, “The effect of carbon distribution on the manganese magnetic moment in bcc Fe-Mn alloy," J. Phys.: Condens. Matter 23, 326003 (2011).

${ }^{219}$ M. Hudl, L. Häggström, E.-K. Delczeg-Czirjak, V. Höglin, M. Sahlberg, L. Vitos, O. Eriksson, P. Nordblad, and Y. Andersson, "Strongly enhanced magnetic moments in ferromagnetic $\mathrm{FeMnP}_{0.5} \mathrm{Si}_{0.5}$," Appl. Phys. Lett. 99, 152502 (2011).

${ }^{220}$ L. F. Kiss, T. Kemeny, J. Bednarcik, J. Kamarad, Z. Arnold, Z. Konopkova, and H.-P. Liermann, "Pressure dependence of magnetic properties in Fe-Mn-B amorphous alloys: Evidence for inhomogeneous ferromagnetism,” J. Phys.: Condens. Matter 25, 346002 (2013).

${ }^{221}$ I. Galanakis, P. Mavropoulos, and P. H. Dederichs, "Electronic structure and Slater-Pauling behaviour in half-metallic Heusler alloys calculated from first principles," J. Phys. D: Appl. Phys. 39, 765 (2006).

${ }^{222}$ J. M. D. Coey, "The magnetization of bulk alpha $\mathrm{Fe}_{16} \mathrm{~N}_{2}$ (invited)," J. Appl. Phys. 76, 6632 (1994).

${ }^{223}$ G. W. Fernando, R. E. Watson, M. Weinert, A. N. Kocharian, A. Ratnaweera, and K. Tennakone, "Magnetic moment of iron in metallic environments," Phys. Rev. B 61, 375-381 (2000).

${ }^{224}$ T. K. Kim and M. Takahashi, "New magnetic material having ultrahigh magnetic moment,” Appl. Phys. Lett. 20, 492 (1972).

${ }^{225}$ M. Komuro, Y. Kozono, M. Hanazono, and Y. Sugita, "Epitaxial growth and magnetic properties of $\mathrm{Fe}_{16} \mathrm{~N}_{2}$ films with high saturation magnetic flux density (invited), ' J. Appl. Phys. 67, 5126-5130 (1990).

${ }^{226}$ Y. Sugita, K. Mitsuoka, and M. Komuro, "Magnetic properties of $\mathrm{Fe}_{16} \mathrm{~N}_{2}$ Films,” IEEE Transl. J. Magn. Jpn. 6(12), 1061-1073 (1991).

${ }^{227}$ H. Takahashi, K. Mitsuoka, M. Komuro, and Y. Sugita, "Ferromagnetic resonance studies of $\mathrm{Fe}_{16} \mathrm{~N}_{2}$ films with a giant magnetic moment," J. Appl. Phys. 73, 6060-6062 (1993).

${ }^{228}$ Y. Sugita, H. Takahashi, M. Komuro, K. Mitsuoka, and A. Sakuma, "Magnetic and Moessbauer studies of single-crystal $\mathrm{Fe}_{16} \mathrm{~N}_{2}$ and $\mathrm{Fe}-\mathrm{N}$ martensite films epitaxially grown by molecular beam epitaxy (invited)," J. Appl. Phys. 76(10), 6637 (1994).

${ }^{229}$ R. M. Metzger and X. Bao, "Magnetism of alpha- $\mathrm{Fe}_{16} \mathrm{~N}_{2}$ (invited)," J. Appl. Phys. 76(10), 6626 (1994).

${ }^{230}$ A. S. Arrott, "Resolution of conflicts concerning $\mathrm{Fe}_{16} \mathrm{~N}_{2}$ (abstract)," J. Appl. Phys. 79, 4570-4570 (1996).
${ }^{231}$ J. M. Cadogan, "Are there giant magnetic moments in Fe-nitrides?," Aust. J. Phys. 50, 1093-1102 (1997).

${ }^{232}$ K. H. Jack, “ $\alpha$ " $\mathrm{Fe}_{16} \mathrm{~N}_{2}$ : A giant magnetic moment material?," Mater. Sci. Forum 325-326, 91-98 (2000).

${ }^{233}$ M. Takahashi and H. Shoji, " $\alpha{ }^{\prime \prime}-\mathrm{Fe}_{16} \mathrm{~N}_{2}$ : Giant magnetic moment or not?,” Philos. Mag. B 80(2), 215 (2000).

${ }^{234}$ X. Zhang, M. Yang, Y. Jiang, L. F. Allard, and J.-P. Wang, "Thermal stability of partially ordered $\mathrm{Fe}_{16} \mathrm{~N}_{2}$ film on non-magnetic Ag under layer," J. Appl. Phys. 115, 17A767 (2014).

${ }^{235}$ R. Coehoorn, G. H. O. Daalderop, and H. J. F. Jansen, "Full-potential calculations of the magnetization of $\mathrm{Fe}_{16} \mathrm{~N}_{2}$ and $\mathrm{Fe}_{4} \mathrm{~N}$," Phys. Rev. B: Condens. Matter 48, 3830-3834 (1993).

${ }^{236}$ H. Tanaka, H. Harima, T. Yamamoto, H. Katayama-Yoshida, Y. Nakata, and Y. Hirotsu, "Electronic band structure and magnetism of $\mathrm{Fe}_{16} \mathrm{~N}_{2}$ calculated by the FLAPW method," Phys. Rev. B 62, 15042-15046 (2000).

${ }^{237}$ Y. Chen, Q. Song, H. Yan, T. Wei, and X. Yang, "Volume dependence of electronic structure and magnetic properties of $\mathrm{Fe}_{16} \mathrm{~N}_{2}$," Phys. B: Condens. Matter 407, 519-522 (2012).

${ }^{238}$ H. Sawada, A. Nogami, and T. Matsumiya, "Electronic structure analysis of magnetic properties of $\mathrm{Fe}_{16} \mathrm{~N}_{2}$," J. Comput.-Aided Mater. Des. 1, 75-84 (1993).

${ }^{239}$ J. He, Y. Zhou, W. Lai, and C. Pan, "Using generalized gradient approximation method to study the electronic structure of ferromagnetic nitride $\mathrm{Fe}_{16} \mathrm{~N}_{2}$," IEEE Trans. Magn. 31, 3668-3670 (1995).

${ }^{240}$ S. Wang and M. H. Kryder, "Rf-diode-sputtered iron nitride films for thin-film recording head materials," J. Appl. Phys. 67, 5134-5136 (1990).

${ }^{241}$ Y. Hoshi and M. Naoe, "Deposition of Fe-N films by means of an opposed targets sputtering type plasma source," J. Appl. Phys. 69, 5622-5624 (1991).

${ }^{242}$ D. H. Mosca, S. R. Teixeira, P. H. Dionisio, I. J. R. Baumvol, W. H. Schreiner, and W. A. Monteiro, "The evolution of sputtered iron nitride thin films under thermal treatment,” J. Appl. Phys. 69, 261-267 (1991).

${ }^{243}$ K. Kusumi, H. Miyajima, and Y. Otani, "Compositional variation of saturation magnetization for $\mathrm{Fe}-\mathrm{N}$ thin films with respect to nitrogen," IEEE Transl. J. Magn. Jpn. 9, 191-196 (1994).

${ }^{244}$ M. Takahashi, H. Shoji, H. Takahashi, H. Nashi, T. Wakiyama, M. Doi, and M. Matsui, "Magnetic moment of alpha- $\mathrm{Fe}_{16} \mathrm{~N}_{2}$ films (invited)," J. Appl. Phys. 76(10), 6642 (1994).

${ }^{245}$ E. Y. Jiang, D. C. Sun, C. Lin, M. B. Tian, H. L. Bai, and S. L. Ming, "Facing targets sputtered Fe-N gradient films," J. Appl. Phys. 78, 2596-2600 (1995).

${ }^{246}$ M. A. Brewer, K. M. Krishnan, and C. Ortiz, "Epitaxial $\mathrm{Fe}_{16} \mathrm{~N}_{2}$ films grown on $\mathrm{Si}(001)$ by reactive sputtering," J. Appl. Phys. 79, 5321 (1996).

${ }^{247}$ L. A. Chebotkevich, Y. D. Vorob'ev, and I. V. Pisarenko, "Magnetic properties of iron nitride films obtained by reactive magnetron sputtering," Phys. Solid State 40, 650-651 (1998).

${ }^{248}$ S. C. Byeon, F. Liu, and G. J. Mankey, "High moment epitaxial Fe-N thin films," IEEE Trans. Magn. 37(4), 1770 (2001).

${ }^{249}$ N. D. Telling, G. A. Jones, P. J. Grundy, and H. J. Blythe, "Fe-N alloy films prepared using a nitrogen atom source," J. Magn. Magn. Mater. 226-230, 1659-1661 (2001).

${ }^{250}$ H. Wang, S. Mitani, H. Fujimori, and M. Motokawa, "Preferential growth of $\alpha^{\prime}$-FeN films under a high magnetic field," Jpn. J. Appl. Phys., Part 2 41, L1075 (2002).

${ }^{251}$ X. Wang, W. T. Zheng, H. W. Tian, S. S. Yu, W. Xu, S. H. Meng, X. D. He, J. C. Han, C. Q. Sun, and B. K. Tay, "Growth, structural, and magnetic properties of iron nitride thin films deposited by dc magnetron sputtering," Appl. Surf. Sci. 220, 30-39 (2003).

${ }^{252}$ M. A. Abdellateef, C. Heiden, H. Lemke, F. M. El-Hossary, and K. Baerner, "Magnetic properties and structure of the $\alpha^{\prime \prime}-\mathrm{Fe}_{16} \mathrm{~N}_{2}$ films," J. Magn. Magn. Mater. 256, 214-220 (2003).

${ }^{253}$ W. H. Zhong, B. K. Tay, S. P. Lau, X. W. Sun, S. Li, and C. Q. Sun, "Structural and magnetic properties of iron-nitride thin films deposited using a filtered cathodic vacuum arc," Thin Solid Films 478, 61-66 (2005).

${ }^{254}$ S. Atiq, H.-S. Ko, S. A. Siddiqi, and S.-C. Shin, "Preparation and the influence of $\mathrm{Co}$, Pt and $\mathrm{Cr}$ additions on the saturation magnetization of $\alpha^{\prime \prime}-\mathrm{Fe}_{16} \mathrm{~N}_{2}$ thin films," J. Alloys Compd. 479, 755-758 (2009).

${ }^{255}$ X. P. Feng, W. B. Mi, and H. L. Bai, "Investigation of structure and magnetic properties of the as-deposited and post-annealed iron nitride films by reactive facing-target sputtering," Appl. Surf. Sci. 257, 7320-7325 (2011).

${ }^{256}$ W. B. Mi, X. P. Feng, and H. L. Bai, "Magnetic properties and Hall effect of reactive sputtered iron nitride nanocrystalline films," J. Magn. Magn. Mater. 323, 1909-1913 (2011). 
${ }^{257}$ Y.-C. Lin, J.-Y. Hong, C.-N. Yen, S.-Y. Tong, M.-J. Tung, H.-W. Shiu, C.-H. Chen, and M.-T. Lin, "X-ray photoelectron spectroscopic investigation on Fe geometrical sites of iron nitride thin films," Jpn. J. Appl. Phys., Part 1 54, 033002 (2015).

${ }^{258}$ X. Li, J. Du, X. Sun, J. Wang, and Q. Liu, "Magnetic properties of iron nitride films prepared by oblique sputtering under different nitrogen gas flow ratios $\left(\mathrm{N}_{2} / \mathrm{N}_{2}+\mathrm{Ar}\right)$," J. Phys. Chem. Solids 85, 13-17 (2015).

${ }^{259}$ I. Dirba, P. Komissinskiy, O. Gutfleisch, and L. Alff, "Increased magnetic moment induced by lattice expansion from $\alpha-\mathrm{Fe}$ to $\alpha^{\prime}-\mathrm{Fe}_{8} \mathrm{~N}$," J. Appl. Phys. 117, 173911 (2015)

${ }^{260}$ S. Okamoto, O. Kitakami, and Y. Shimada, " $\alpha$ " $-\mathrm{Fe}_{16} \mathrm{~N}_{2}$ phase epitaxially grown by sputter beam method," J. Appl. Phys. 79, 5250-5252 (1996).

${ }^{261}$ Z.-Y. Yao, H. Jiang, Z.-K. Liu, D.-D. Huang, F.-G. Qin, S.-C. Zhu, and Y.-X. Sun, "Formation and magnetic properties of $\mathrm{Fe}_{16} \mathrm{~N}_{2}$ films prepared by ion-beam-assisted deposition," J. Magn. Magn. Mater. 177-181, 1291-1292 (1998)

${ }^{262}$ S. Iwatsubo, "Preparation of FeN films deposited by dual ion beam sputtering and properties after annealing," J. Magn. Soc. Jpn. 25, 863-866 (2001).

${ }^{263}$ K. Nakajima and S. Okamoto, "Nitrogen-implantation-induced transformation of iron to crystalline $\mathrm{Fe}_{16} \mathrm{~N}_{2}$ in epitaxial iron films," Appl. Phys. Lett. 54, 2536-2538 (1989).

${ }^{264}$ T. Weber, L. de Wit, F. W. Saris, and P. Schaaf, "Search for giant magnetic moments in ion-beam-synthesized $\alpha^{\prime \prime}-\mathrm{Fe}_{16} \mathrm{~N}_{2}$," Thin Solid Films 279, 216-220 (1996).

${ }^{265} \mathrm{H}$. Shinno and K. Saito, "Effects of film thickness on formation processes of $\mathrm{Fe}_{16} \mathrm{~N}_{2}$ in nitrogen ion-implanted Fe films," Surf. Coat. Technol. 103-104, 129-134 (1998).

${ }^{266}$ M. Gupta, A. Gupta, P. Bhattacharya, P. Misra, and L. M. Kukreja, "Study of iron nitride thin films deposited by pulsed laser deposition," J. Alloys Compd. 326, 265-269 (2001).

${ }^{267}$ N. Wang, K. M. Ulmer, A. P. Constant, J. W. Anderegg, and J. E. Snyder, "Observation of the phase formation in Fe-N films deposited by reactive pulsed laser deposition,” J. Vac. Sci. Technol. A 21, 1734-1738 (2003).

${ }^{268}$ Y. D. Zhang, J. I. Budnick, W. A. Hines, M. Q. Huang, and W. E. Wallace, "Giant moment of $\mathrm{Fe}_{16} \mathrm{~N}_{2}$ as evidenced by ${ }^{57} \mathrm{Fe}$ NMR studies," Phys. Rev. B 54, 51-54 (1996).

${ }^{269}$ K. H. Jack, "The occurrence and the crystal structure of $\alpha$ " iron nitride; a new type of interstitial alloy formed during the tempering of nitrogenmartensite," Proc. R. Soc. Lond. A 208, 216-224 (1951).

${ }^{270}$ T. Takahashi, N. Takahashi, T. Nakamura, T. Kato, K. Furukawa, G. M. Smith, and P. C. Riedi, "Magnetic characteristics of $\mathrm{Fe}_{4} \mathrm{~N}$ epitaxial films grown by halide vapor phase deposition under atmospheric pressure," Solid State Sci. 6, 97-99 (2004)

${ }^{271}$ E. Kita, K. Shibata, H. Yanagihara, Y. Sasaki, and M. Kishimoto, "Magnetic properties of core-shell type nanoparticles," J. Magn. Magn. Mater. 310, 2411-2413 (2007).

${ }^{272}$ S. Yamashita, Y. Masubuchi, Y. Nakazawa, T. Okayama, M. Tsuchiya, and S. Kikkawa, "Crystal structure and magnetic properties of $\alpha^{\prime \prime}-\mathrm{Fe}_{16} \mathrm{~N}_{2}$ containing residual $\alpha$-Fe prepared by low-temperature ammonia nitridation," J. Solid State Chem. 194, 76-79 (2012).

${ }^{273}$ T. Ogawa, Y. Ogata, R. Gallage, N. Kobayashi, N. Hayashi, Y. Kusano, S. Yamamoto, K. Kohara, M. Doi, M. Takano, and M. Takahashi, "Challenge to the synthesis of $\alpha^{\prime \prime}-\mathrm{Fe}_{16} \mathrm{~N}_{2}$ compound nanoparticle with high saturation magnetization for rare earth free new permanent magnetic material," Appl. Phys. Express 6, 073007 (2013).

${ }^{274}$ K. Takagi, M. Akada, K. Ozaki, N. Kobayashi, T. Ogawa, Y. Ogata, and M. Takahashi, "High-pressure sintering behavior of $\alpha^{\prime \prime}-\mathrm{Fe}_{16} \mathrm{~N}_{2}$ nanopowder,” J. Appl. Phys. 115, 103905 (2014).

${ }^{275}$ S. Bhattacharyya, "Iron nitride family at reduced dimensions: A review of their synthesis protocols and structural and magnetic properties," J. Phys. Chem. C 119, 1601-1622 (2015).

${ }^{276}$ R. Zulhijah, A. B. D. Nandiyanto, T. Ogi, T. Iwaki, K. Nakamura, and K. Okuyama, "Effect of oxidation on $\alpha^{\prime \prime}-\mathrm{Fe}_{16} \mathrm{~N}_{2}$ phase formation from plasma-synthesized spherical core-shell $\alpha-\mathrm{Fe} / \mathrm{Al}_{2} \mathrm{O}_{3}$ nanoparticles," J. Magn. Magn. Mater. 381, 89-98 (2015).

${ }^{277}$ L. Ke, K. D. Belashchenko, M. van Schilfgaarde, T. Kotani, and V. P. Antropov, "Effects of alloying and strain on the magnetic properties of $\mathrm{Fe}_{16} \mathrm{~N}_{2}$," Phys. Rev. B 88, 024404 (2013).

${ }^{278}$ C. Ortiz, G. Dumpich, and A. H. Morrish, "Epitaxial $\mathrm{Fe}_{16} \mathrm{~N}_{2}$ films grown by sputtering," Appl. Phys. Lett. 65, 2737-2739 (1994).

${ }^{279}$ D. C. Sun, C. Lin, and E. Y. Jiang, "Epitaxially grown $\mathrm{Fe}_{16} \mathrm{~N}_{2}$ singlecrystal films with high saturation magnetization prepared by facing targets sputtering,” J. Phys.: Condens. Matter 7, 3667 (1995).
${ }^{280}$ D. C. Sun, E. Y. Jiang, M. B. Tian, C. Lin, and X. X. Zhang, "Epitaxial single crystal $\mathrm{Fe}_{16} \mathrm{~N}_{2}$ films grown by facing targets sputtering," J. Appl. Phys. 79, 5440-5442 (1996).

${ }^{281}$ K. M. Krishnan, M. A. Brewer, T. Kobayashi, A. Nakanishi, and A. Young, "Giant moment in sputtered Fe-N films grown epitaxially on Si(001)," in Magnetic Hysteresis in Novel Magnetic Materials, NATO ASI Series, edited by G. C. Hadjipanayis (Springer, Netherlands, 1997), Vol. 338, pp. 561-565.

${ }^{282}$ S. Kikkawa, M. Fujiki, S. Enomoto, K.-i. Hirota, and O. Yamaguchi, "Giant magnetism in Fe metal/AIN multilayered thin film prepared by RF-sputter deposition," Mater. Sci. Forum 325-326, 111-116 (2000).

${ }^{283}$ G. Pan, I. Wang, R. Buckley, K. Kang, and A. Petford-Long, "Reactive sputtering of high moment Fe-N soft magnetic films with in-situ plasma diagnosis and control," IEEE Trans. Magn. 37, 2284-2287 (2001).

${ }^{284}$ Y. F. Chen, E. Y. Jiang, Z. Q. Li, W. B. Mi, P. Wu, and H. L. Bai, "Structure and magnetic properties of RF sputtered Fe-N films," J. Phys. D: Appl. Phys. 37, 1429 (2004).

${ }^{285}$ N. Ji, L. F. Allard, E. Lara-Curzio, and J.-P. Wang, "N site ordering effect on partially ordered $\mathrm{Fe}_{16} \mathrm{~N}_{2}$," Appl. Phys. Lett. 98(9), 092506 (2011).

${ }^{286}$ J.-P. Wang, N. Ji, X. Liu, Y. Xu, C. Sanchez-Hanke, Y. Wu, F. de Groot, L. Allard, and E. Lara-Curzio, "Fabrication of films by sputtering process and experimental investigation of origin of giant saturation magnetization in $\mathrm{Fe}_{16} \mathrm{~N}_{2}$," IEEE Trans. Magn. 48, 1710-1717 (2012).

${ }^{287}$ K. Mitsuoka, H. Miyajima, H. Ino, and S. Chikazumi, "Induced magnetic moment in ferromagnetic Fe alloys by tetragonally elongated lattice expansion," J. Phys. Soc. Jpn. 53, 2381-2390 (1984).

${ }^{288}$ J. M. D. Coey and P. A. I. Smith, "Magnetic nitrides," J. Magn. Magn. Mater. 200, 405-424 (1999).

${ }^{289}$ M. B. Tian, E. Y. Jiang, and D. C. Sun, "Fe-N gradient films and epitaxial $\mathrm{Fe}_{16} \mathrm{~N}_{2}$ single-crystal films," J. Vac. Sci. Technol. A 15, 2313-2317 (1997).

${ }^{290}$ S. R. Kappaganthu and Y. Sun, "Effect of nitrogen partial pressure and temperature on RF sputtered Fe-N films," Surf. Coat. Technol. 167, 165-169 (2003).

${ }^{291}$ N. Ji, Y. Wu, and J.-P. Wang, "Epitaxial high saturation magnetization FeN thin films on $\mathrm{Fe}(001)$ seeded $\mathrm{GaAs}(001)$ single crystal wafer using facing target sputterings," J. Appl. Phys. 109, 07 B767 (2011).

${ }^{292}$ S. Malinov, A. J. Böttger, E. J. Mittemeijer, M. I. Pekelharing, and M. A. J. Somers, "Phase transformations and phase equilibria in the Fe-N system at temperatures below $573 \mathrm{~K}$," Metall. Mater. Trans. A 32, 59-73 (2001).

${ }^{293}$ M. Widenmeyer, T. C. Hansen, and R. Niewa, "Formation and decomposition of metastable $\alpha^{\prime \prime}-\mathrm{Fe}_{16} \mathrm{~N}_{2}$ from in situ powder neutron diffraction and thermal analysis," Z. Anorg. Allg. Chem. 639, 2851-2859 (2013).

${ }^{294}$ T. Koyano, T. Nomiyama, N. Kanoh, H. Numata, T. Ohba, E. Kita, and H. Ohtsuka, "Magnetization of $\alpha$ iron nitride produced through the fcc bct martensitic transformation in high magnetic field," J. Appl. Phys. 100, 033906 (2006).

${ }^{295}$ N. Ji, X. Liu, and J.-P. Wang, “Theory of giant saturation magnetization in $\alpha-\mathrm{Fe}_{16} \mathrm{~N}_{2}$ : Role of partial localization in ferromagnetism of $3 \mathrm{~d}$ transition metals," New J. Phys. 12, 063032 (2010).

${ }^{296}$ H. Sims, W. H. Butler, M. Richter, K. Koepernik, E. Şaşioğlu, C. Friedrich, and S. Blügel, "Theoretical investigation into the possibility of very large moments in $\mathrm{Fe}_{16} \mathrm{~N}_{2}$," Phys. Rev. B 86, 174422 (2012).

${ }^{297}$ Y. Shi, Y. Du, and G. Chen, "Correlation of electronic structure and magnetic moment in $\mathrm{Fe}_{16} \mathrm{~N}_{2}$ : First-principles calculations," Scr. Mater. 68, 976-979 (2013).

${ }^{298}$ X. Liu, Y.-H. Xu, C. Sanchez-Hanke, and J.-P. Wang, "Discovery of localized states of $\mathrm{Fe} 3 \mathrm{~d}$ electrons in $\mathrm{Fe}_{16} \mathrm{~N}_{2}$ and $\mathrm{Fe}_{8} \mathrm{~N}$ films: An evidence of the existence of giant saturation magnetization," e-print arXiv:0909.4478 [cond-mat].

${ }^{299}$ N. Ji, V. Lauter, X. Zhang, H. Ambaye, and J.-P. Wang, "Strain induced giant magnetism in epitaxial $\mathrm{Fe}_{16} \mathrm{~N}_{2}$ thin film," Appl. Phys. Lett. 102, $072411(2013)$

${ }^{300}$ X. Zhang, N. Ji, V. Lauter, H. Ambaye, and J.-P. Wang, "Strain effect of multilayer FeN structure on GaAs substrate," J. Appl. Phys. 113, 17 E149 (2013).

${ }^{301}$ M. Yang, L. F. Allard, N. Ji, X. Zhang, G.-H. Yu, and J.-P. Wang, "The effect of strain induced by $\mathrm{Ag}$ underlayer on saturation magnetization of partially ordered $\mathrm{Fe}_{16} \mathrm{~N}_{2}$ thin films," Appl. Phys. Lett. 103, 242412 (2013).

${ }^{302}$ Y. Jiang, V. Dabade, M. P. Brady, O. Rios, R. D. James, and J.-P. Wang, "9 T high magnetic field annealing effects on FeN bulk sample," J. Appl. Phys. 115, 17A758 (2014). 
${ }^{303}$ H. Y. Wang, Z. W. Ma, Y. J. He, H. Chen, and E. Y. Jiang, "The effect of nitrogen pressure on the soft magnetic properties of FeTiN films," Phys. Status Solidi A 168(1), 273-280 (1998).

${ }^{304}$ H. Y. Wang, Y. J. He, Z. W. Ma, E. Y. Jiang, H. S. Huang, and W. H. Mao, "High moment soft magnetic FeTiN thin films for recording head materials," J. Appl. Phys. 85, 3745-3748 (1999).

${ }^{305}$ S. C. Byeon, Y. Ding, and C. Alexander, "High moment FeTiN thin films for high density recording heads," IEEE Trans. Magn. 36, 2502-2505 (2000).

${ }^{306}$ R. Gupta, A. Tayal, S. M. Amir, M. Gupta, A. Gupta, M. Horisberger, and J. Stahn, "Formation of iron nitride thin films with $\mathrm{Al}$ and Ti additives," J. Appl. Phys. 111, 103520 (2012).

${ }^{307}$ H. Y. Wang, Jun-Liu, H. S. Huang, W. H. Mao, H. Chen, H. Y. Zhang, Y. J. He, and E. Y. Jiang, "The structure and magnetic properties of FeNiN films," J. Appl. Phys. 91, 1453-1457 (2002).

${ }^{308}$ P. Prieto, K. R. Pirota, J. M. Sanz, E. Jiménez, J. Camarero, F. Maccherozzi, and F. G. Panaccione, "Growth, structural, and magnetic characterizations of nanocrystalline gamma'-FeNiN(220) thin films," Appl. Phys. Lett. 90, 032505 (2007).

${ }^{309}$ H. Y. Wang, Z. W. Ma, E. Y. Jiang, Y. J. He, and H. S. Huang, "Effect of annealing on structure and magnetic properties of $\mathrm{Fe}-\mathrm{N}$ and $\mathrm{Fe}-\mathrm{Ti}-\mathrm{N}$ thin films," Appl. Phys. A 68, 559-562 (1999).

${ }^{310}$ H. Y. Wang and E. Y. Jiang, " $(\mathrm{Fe}, \mathrm{Co}){ }_{16} \mathrm{~N}_{2}$ films with high saturation magnetization prepared by facing target sputtering," J. Phys.: Condens. Matter 9, 8547 (1997).

${ }^{311}$ H. Y. Wang and E. Y. Jiang, "Structure and magnetic properties of FeCoN films," Appl. Phys. A 65, 203-206 (1997).

${ }^{312}$ Y. Liu, Z. W. Liu, C. Y. Tan, and C. K. Ong, "High frequency characteristics of FeCoN thin films fabricated by sputtering at various $\left(\mathrm{Ar}+\mathrm{N}_{2}\right)$ gas flow rates,” J. Appl. Phys. 100, 093912 (2006).

${ }^{313}$ M. Takahashi, H. Takahashi, H. Nashi, H. Shoji, T. Wakiyama, and M. Kuwabara, "Structure and magnetic moment of $\alpha^{\prime \prime}-\mathrm{Fe}_{16} \mathrm{~N}_{2}$ compound films: Effect of $\mathrm{Co}$ and $\mathrm{H}$ on phase formation (invited)," J. Appl. Phys. 79, 5564-5569 (1996).

${ }^{314}$ Y. Inoue, S. Takebayashi, and T. Mukai, "Enhancement of the formation of $\mathrm{Fe}_{16} \mathrm{~N}_{2}$ on $\mathrm{Fe}$ films by $\mathrm{Co}$ additions (invited)," J. Appl. Phys. 76, 6653-6655 (1994).

${ }^{315}$ K. H. Jack, "The synthesis and characterization of bulk $\alpha^{\prime \prime}-\mathrm{Fe}_{16} \mathrm{~N}_{2}$," J. Alloys Compd. 222, 160-166 (1995).

${ }^{316} \mathrm{~B}$. Min, "Enhancement of $\mathrm{Fe}$ magnetic moments in ferromagnetic $\mathrm{Fe}_{16} \mathrm{~B}_{2}$, $\mathrm{Fe}_{16} \mathrm{C}_{2}$, and $\mathrm{Fe}_{16} \mathrm{~N}_{2}$," Int. J. Mod. Phys. B 07, 729-732 (1993).

${ }^{317}$ A. Leineweber, H. Jacobs, F. Hüning, H. Lueken, and W. Kockelmann, "Nitrogen ordering and ferromagnetic properties of $\varepsilon-\mathrm{Fe}_{3} \mathrm{~N}_{1+\mathrm{x}}$ $(0.10 \leq x \leq 0.39)$ and $\varepsilon-\mathrm{Fe}_{3}\left(\mathrm{~N}_{0.80} \mathrm{C}_{0.20}\right)_{1.38}$," J. Alloys Compd. 316, 21-38 (2001).

${ }^{318}$ M. A. Willard, "Stronger, lighter, and more energy efficient: Challenges of magnetic material development for vehicle electrification," in Frontiers of Engineering: Reports on Leading-Edge Engineering from the 2012 Symposium, edited by K. Anseth, P. Boinodiris, K. Burg, and L.-T. Cheng, Frontiers of Engineering (National Academies Press, Washington DC, USA, 2013), p. 57; http://www.nap.edu/read/18185/ chapter/11.

${ }^{319}$ H. Takahashi, M. Igarashi, A. Kaneko, H. Miyajima, and Y. Sugita, "Perpendicular uniaxial magnetic anisotropy of $\mathrm{Fe}_{16} \mathrm{~N}_{2}$ (001) single crystal films grown by molecular beam epitaxy," IEEE Trans. Magn. 35, 2982-2984 (1999).

${ }^{320}$ K. Shibata, Y. Sasaki, M. Kishimoto, H. Yanagihara, and E. Kita, "Magnetic moment and anisotropy of iron nitride $\mathrm{Fe}_{16} \mathrm{~N}_{2}$ nanoparticles," J. Magn. Soc. Jpn. 30(5), 501-504 (2006).

${ }^{321}$ S. Yamamoto, R. Gallage, Y. Ogata, Y. Kusano, N. Kobayashi, T. Ogawa, N. Hayashi, K. Kohara, M. Takahashi, and M. Takano, "Quantitative understanding of thermal stability of $\alpha^{\prime \prime}-\mathrm{Fe}_{16} \mathrm{~N}_{2}$," Chem. Commun. 49, 7708-7710 (2013).

${ }^{322}$ K. Yamaguchi, T. Yui, Y. Ichikawa, K. Yamaki, I. Kakeya, K. Kadowaki, and T. Suemasu, "Epitaxial growth and magnetic properties of ferromagnetic $\mathrm{Fe}_{3} \mathrm{~N}$ on $\mathrm{Si}(111)$ by molecular beam epitaxy using AlN/ 3C-SiC intermediate layers,” Jpn. J. Appl. Phys., Part 2 45, L705 (2006).

${ }^{323}$ K. Ito, G. H. Lee, and T. Suemasu, "Epitaxial growth of ferromagnetic $\mathrm{Fe}_{4} \mathrm{~N}$ thin films on $\mathrm{SrTiO}_{3}(001)$ substrates by molecular beam epitaxy," J. Phys.: Conf. Ser. 266(1), 012091 (2011).

${ }^{324}$ J. Jensen and A. R. Mackintosh, Rare Earth Magnetism - Structures and Excitations (Clarendon Press, Oxford, 1991); available at http://www.fys. ku.dk/ jjensen/REM.htm.
${ }^{325}$ F. A. Cotton, G. Wilkinson, C. A. Murillo, and M. Bochmann, Advanced Inorganic Chemistry, 6th ed. (John Wiley \& Sons, Inc., New York, USA, 1999).

${ }^{326}$ J. J. Rhyne and T. R. McGuire, "Magnetism of rare-earth elements, alloys, and compounds," IEEE Trans. Magn. 8(1), 105 (1972).

${ }^{327}$ K. P. Belov, R. Z. Levitin, and S. A. Nikitin, "Ferromagnetism and antiferromagnetism of rare-earth metals," Sov. Phys. - Usp. 7, 179 (1964).

${ }^{328}$ R. W. Hoard, S. C. Mance, R. L. Leber, E. N. Dalder, M. R. Chaplin, K. Blair, D. H. Nelson, and D. A. Van Dyke, "Field enhancement of a 12.5-T magnet using holmium poles," IEEE Trans. Magn. 21(2), 448 (1985).

${ }^{329}$ R. Agustsson, Y. C. Chen, T. Grandsaert II, A. Murokh, V. Solovyov, and F. O'Shea, "Praseodymium iron-boron undulator with textured dysprosium poles for compact x-ray FEL applications," in Proceedings of IPAC2012, New Orleans, Lousiana, USA (2012), vol. MOPPP086, p. 756; available at https://accelconf.web.cern.ch/accelconf/IPAC2012/ papers/moppp086.pdf.

${ }^{330} \mathrm{~F}$. Milstein and L. B. Robinson, "Magnetic transitions in alloys of gadolinium and dysprosium," Phys. Rev. 159(2), 466 (1967).

${ }^{331}$ T.-T. Yang and L. B. Robinson, "Magnetic resistivity and magnetic transitions in rare-earth alloys," Phys. Rev. 185(2), 743 (1969).

${ }^{332}$ R. Z. Levitin, T. M. Perekalina, L. P. Shlyakhina, O. D. Chistyakov, and V. L. Yakovenko, "Nature of magnetic anisotropy of dysprosium. Paramagnetic susceptibility of dysprosium-gadolinium alloys," Sov. Phys. JETP 36(4), 742 (1973); available at http://www.jetp.ac.ru/cgi-bin/ e/index/e/36/4/p742?a=list.

${ }^{333}$ W. Dai, J. Yang, B. Shen, D. Li, and Z. Gao, "Effect of introduction of light rare-earth into heavy ones on magnetic properties of the alloys," J. Mater. Sci. Technol. 13, 293 (1997); available at http://www.jmst.org/ EN/abstract/abstract14895.shtml.

${ }^{334}$ W. C. Koehler, "Magnetic properties of rare-earth metals and alloys," J. Appl. Phys. 36, 1078 (1965).

${ }^{335}$ R. M. Bozorth, R. J. Gambino, and A. E. Clark, "Magnetization of some single-crystal alloys of rare earth metals," J. Appl. Phys. 39(2), 883 (1968).

${ }^{336}$ R. M. Bozorth, "Magnetic properties of compounds and solid solutions of rare-earth metals," J. Appl. Phys. 38(3), 1366 (1967).

${ }^{337}$ A. Y. Amin, "Magnetic properties of inter-rare-earth alloys at low temperatures in high magnetic fields," J. Appl. Phys. 50(2), 1035 (1979).

${ }^{338}$ K. P. Belov, O. P. Iliotin, S. A. Nikitin, G. V. Pshechenkova, V. E. Sokolov, and V. P. Taratynov, "Dy-Er magnetic alloys," UDSSR patent SU276424(A1) (1970).

${ }^{339}$ K. P. Belov, O. P. Iliotin, S. A. Nikitin, G. V. Pshechenkova, V. E. Sokolov, and V. P. Taratynov, "Ho-Er magnetic alloys," UDSSR patent SU276423(A1) (1970).

${ }^{340}$ O. P. Elyutin, G. V. Pshechenkova, V. P. Taratynov, and L. A. Shul'te, "Effect of erbium on magnetization of Ho-Dy alloys," Met. Sci. Heat Treat. 15(2), 113 (1973).

${ }^{341}$ R. A. Cowley, C. Bryn-Jacobsen, J. A. Simpson, D. F. McMorrow, R. C. C. Ward, and M. R. Wells, "The unusual phase diagram of the magnetic structures of holmium-erbium alloys,” J. Magn. Magn. Mater. 177-181, 1012 (1998).

${ }^{342}$ J. Kwo, M. Hong, D. B. McWhan, Y. Yafet, R. M. Fleming, F. J. DiSalvo, J. V. Waszczak, C. F. Majkrzak, D. Gibbs, A. I. Goldman, P. Boni, J. Bohr, H. Grimm, C. L. Chien, and J. W. Cable, "Magnetic superlattices," J. Phys. C8(49), 1651 (1988).

${ }^{343}$ C. F. Majkrzak, J. Kwo, M. Hong, Y. Yafet, D. Gibbs, C. L. Chien, and J. Bohr, "Magnetic rare earth superlattices," Adv. Phys. 40, 99 (1991).

${ }^{344}$ K. Dumesnil, C. Dufour, P. Mangin, G. Marchal, and H. Hennion, "Magnetic structure of dysprosium in epitaxial Dy films and in Dy/Er superlattices," Phys. Rev. B 54, 6407 (1996).

${ }^{345}$ R. S. Beach, A. Matheny, M. B. Salamon, C. P. Flynn, J. A. Borchers, R. W. Erwin, and J. J. Rhyne, "Magnetism and epitaxy in $\mathrm{Lu} / \mathrm{Dy} / \mathrm{Lu}$ trilayers," J. Appl. Phys. 73, 6901 (1993).

${ }^{346}$ M. C. Luche, A. Baudry, P. Boyer, J. L. Rouviere, C. Fermon, and C. Miramond, "Structure and magnetism of dysprosium/zirconium multilayers,” J. Magn. Magn. Mater. 150, 175 (1995).

${ }^{347}$ G. Scheunert, W. R. Hendren, A. A. Lapicki, R. Hardeman, M. Gubbins, and R. M. Bowman, "Improved magnetization in sputtered dysprosium thin films," J. Phys. D: Appl. Phys. 46, 152001 (2013).

${ }^{348}$ A. E. Curzon and H. G. Chlebek, "The observation of face centered cubic Gd, Tb, Dy, Ho, Er and Tm in the form of thin films and their oxidation," J. Phys. F: Met. Phys. 3, 1 (1973). 
${ }^{349}$ B. M. S. Bist and O. N. Srivastava, "A new F.C.C. gadolinium phase and its oxidation,” J. Less-Common Met. 33, 99 (1973).

${ }^{350} \mathrm{G}$. Scheunert, C. Ward, W. R. Hendren, and R. M. Bowman, "Magnetization of 2.6T in gadolinium thin films," Appl. Phys. Lett. 101, 142407 (2012).

${ }^{351}$ C. Ward, G. Scheunert, W. R. Hendren, and R. M. Bowman, "Realizing the high moment in $\mathrm{Fe} / \mathrm{Cr} / \mathrm{Gd}$ : The role of the rare earth," Appl. Phys. Lett. 102, 092403 (2013)

${ }^{352}$ T. P. Bertelli, E. C. Passamani, C. Larica, V. P. Nascimento, A. Y. Takeuchi, and M. S. Pessoa, "Ferromagnetic properties of fcc Gd thin films," J. Appl. Phys. 117, 203904 (2015).

${ }^{353}$ A. A. A. Hussain and T. S. Al-Bassam, "Effects of deposition parameters on the structure of thin gadolinium films," Thin Solid Films 59, 77 (1979).

${ }^{354}$ R. Kalinowski, C. Meyer, A. Wawro, and L. T. Baczewski, "Magnetic anisotropy in MBE-grown epitaxial gadolinium ultra-thin films," Thin Solid Films 367, 189 (2000).

${ }^{355}$ A. Heys and P. E. Donovan, "Magnetisation measurements on gadolinium/tungsten multilayers,” J. Magn. Magn. Mater. 126, 326 (1993).

${ }^{356}$ A. Heys, P. Donovan, A. Petford-Long, and R. Cywinski, "Giant magnetic moments in gadolinium/tungsten multilayers," J. Magn. Magn. Mater. 131(1-2), 265-272 (1994).

${ }^{357}$ R. Skomski, C. Waldfried, and P. A. Dowben, "The influence of the surface on the spontaneous magnetization of Gd thin films," J. Phys.: Condens. Matter 10, 5833 (1998).

${ }^{358}$ J. S. Jiang and C. L. Chien, "Magnetization and finite-size effects in Gd/ W multilayers,” J. Appl. Phys. 79(8), 5615 (1996).

${ }^{359}$ Y. Li, C. Polaczyk, J. Kapoor, and D. Riegel, "Reduced magnetization in Gd/W multilayers,” J. Magn. Magn. Mater. 165, 165 (1997).

${ }^{360}$ S. Dhar, O. Brandt, M. Ramsteiner, V. F. Sapega, and K. H. Ploog, “Colossal magnetic moment of Gd in GaN," Phys. Rev. Lett. 94, 037205 (2005).

${ }^{361}$ S. Dhar, T. Kammermeier, A. Ney, L. Perez, K. H. Ploog, A. Melnikov, and A. D. Wieck, "Ferromagnetism and colossal magnetic moment in Gd-focused ion-beam-implanted GaN," Appl. Phys. Lett. 89, 062503 (2006).

${ }^{362}$ A. Ney, T. Kammermeier, E. Manuel, V. Ney, S. Dhar, K. H. Ploog, F. Wilhelm, and A. Rogalev, "Element specific investigations of the structural and magnetic properties of Gd:GaN," Appl. Phys. Lett. 90, 252515 (2007).

${ }^{363}$ J. Hejtmanek, K. Knizek, M. Marysko, Z. Jirak, D. Sedmidubsky, Z. Sofer, V. Perina, H. Hardtdegen, and C. Buchal, "On the magnetic properties of Gd implanted GaN,” J. Appl. Phys. 103, 07 D107 (2008).

${ }^{364}$ A. Ney, T. Kammermeier, V. Ney, S. Ye, K. Ollefs, E. Manuel, S. Dhar, K. H. Ploog, E. Arenholz, F. Wilhelm, and A. Rogalev, "Element specific magnetic properties of Gd-doped GaN: Very small polarization of Ga and paramagnetism of Gd," Phys. Rev. B 77, 233308 (2008).

${ }^{365}$ A. Ney, T. Kammermeier, K. Ollefs, V. Ney, S. Ye, S. Dhar, K. H. Ploog, M. Roever, J. Malindretos, A. Rizzi, F. Wilhelm, and A. Rogalev, "Gddoped GaN studied with element specificity: Very small polarization of $\mathrm{Ga}$, paramagnetism of Gd and the formation of magnetic clusters," J. Magn. Magn. Mater. 322, 1162 (2010).

${ }^{366}$ L. Perez, G. S. Lau, S. Dhar, O. Brandt, and K. H. Ploog, "Magnetic phases and anisotropy in Gd-doped GaN," Phys. Rev. B 74, 195207 (2006).

${ }^{367}$ M. A. Khaderbad, S. Dhar, L. Perez, K. H. Ploog, A. Melnikov, and A. D. Wieck, "Effect of annealing on the magnetic properties of Gd focused ion beam implanted GaN," Appl. Phys. Lett. 91, 072514 (2007).

${ }^{368}$ G. M. Dalpian and S.-H. Wei, "Electron-induced stabilization of ferromagnetism in $\mathrm{Ga}_{(1-\mathrm{X})} \mathrm{Gd}_{(\mathrm{X})} \mathrm{N}$," Phys. Rev. B 72, 115201 (2005).

${ }^{369}$ Y. Gohda and A. Oshiyama, "Intrinsic ferromagnetism due to cation vacancies in Gd-doped GaN: First principle calculations," Phys. Rev. B 78, 161201 (2008)

${ }^{370}$ L. Liu, P. Y. Yu, Z. Ma, and S. S. Mao, "Ferromagnetism in GaN:Gd: A density functional theory study," Phys. Rev. Lett. 100, 127203 (2008).

${ }^{371}$ Z. Sofer, D. Sedmidubský, M. Moram, A. Macková, M. Maryško, J. Hejtmánek, C. Buchal, H. Hardtdegen, M. Václavi̊, V. Peřina, R. Groetzschel, and M. Mikulics, "Magnetism in GaN layers implanted by La, Gd, Dy and Lu," Thin Solid Films 519, 6120-6125 (2011).

${ }^{372}$ G. Zhong, K. Zhang, F. He, X. Ma, L. Lu, Z. Liu, and C. Yang, "Ga vacancy induced ferromagnetism enhancement and electronic structures of RE-doped GaN,” Phys. B: Condens. Matter 407, 3818-3827 (2012).

${ }^{373}$ X. Gao, C. Liu, C. Yin, D. Tao, C. Yang, and B. Man, "Structural, morphological and magnetic characteristics of Tb-implanted GaN and AlGaN films," Mater. Sci. Eng. B 178, 349-353 (2013).
${ }^{374}$ M. Marysko, J. Hejtmanek, V. Laguta, Z. Sofer, D. Sedmidubsky, P. Simek, M. Vesely, M. Mikulics, C. Buchal, A. Mackova, P. Malinsky, and R. A. Wilhelm, "Ferromagnetic and paramagnetic magnetization of implanted GaN:Ho,Tb,Sm,Tm films,” J. Appl. Phys. 117, 17 B907 (2015).

${ }^{375}$ D. M. Zayachuk, "Magnetic susceptibility of the rare earth element impurities in the IV-VI semiconductors and Curie-Weiss law,” J. Magn. Magn. Mater. 322, 60-64 (2010).

${ }^{376}$ W. Xiao, Q. Guo, Q. Xue, and E. G. Wang, "Gd on GaN(0001) surface: Growth, interaction, and Fermi level movement," J. Appl. Phys. 94, 4847-4852 (2003)

${ }^{377}$ V. Stepankin, "Magnetically aligned polycrystalline dysprosium as ultimate saturation ferromagnet for high magnetic field polepieces," Phys. B: Condens. Matter 211, 345-347 (1995).

${ }^{378}$ K. H. J. Buschow, "Intermetallic compounds of rare-earth and $3 \mathrm{~d}$ transition metals," Rep. Prog. Phys. 40(10), 1179 (1977).

${ }^{379}$ K. H. J. Buschow, "Intermetallic compounds of rare earths and nonmagnetic metals,” Rep. Prog. Phys. 42, 1373 (1979).

${ }^{380}$ A. L. Dantas, R. E. Camley, and A. S. Carrico, "Thermal hysteresis of thin Dy films," IEEE Trans. Magn. 42, 2942 (2006).

${ }^{381}$ Z. S. Shan and D. J. Sellmyer, "Structure and magnetic properties of nanostructured Dy/transition-metal multilayered films," J. Appl. Phys. 64, 5745 (1988).

${ }^{382}$ C. F. Majkrzak, D. Gibbs, P. Boeni, A. I. Goldman, J. Kwo, M. Hong, T. C. Hsieh, R. M. Fleming, D. B. McWhan, Y. Yafet, J. W. Cable, J. Bohr, H. Grimm, and C. L. Chien, "Magnetic rare-earth superlattices (invited)," J. Appl. Phys. 63, 3447 (1988).

${ }^{383}$ I. D. Hughes, M. Däne, A. Ernst, W. Hergert, M. Lüders, J. Poulter, J. B. Staunton, A. Svane, Z. Szotek, and W. M. Temmerman, "Lanthanide contraction and magnetism in the heavy rare earth elements," Nature 446, 650-653 (2007).

${ }^{384}$ A. S. Andreenko and S. A. Nikitin, "Magnetic properties of amorphous rare-earth \pm 3d-transition-metal alloys," Phys. - Usp. 40(6), 581 (1997).

${ }^{385}$ B. R. Coles, "The magnetic properties of platinum metals and alloys," Platinum Met. Rev. 8(1), 9 (1964); available at www.technology.matthey. com/pdf/pmr-v8-i1-009-011.pdf.

${ }^{386}$ L. L. Isaacs, D. J. Lam, and F. Y. Fradin, "Magnetic properties of dilute alloys of scandium," J. Appl. Phys. 42, 1458-1459 (1971).

${ }^{387}$ O. Loebich, Jr. and E. Raub, "Das Magnetische Verhalten der Legierungen des Palladiums mit Gadolinium, Dysprosium und Holmium,” J. Less-Common Met. 31, 111 (1973).

${ }^{388}$ O. Loebich, Jr. and E. Raub, “Die legierungen des rutheniums mit gadolinium und dysprosium und ihre magnetischen eigenschaften," J. LessCommon Met. 46, 7-15 (1976).

${ }^{389}$ O. Loebich, Jr. and E. Raub, "Magnetische Eigenschaften der Legierungen des Rhodiums mit Lanthaniden,” Mater. Res. Bull. 10, 1017 (1975).

${ }^{390}$ O. Loebich, Jr. and E. Raub, "Das System Gadolinium-Rhodium und Legierungen des Rhodiums mit anderen Lanthaniden," J. Less-Common Met. 46, 1 (1976).

${ }^{391}$ R. Chamard-Bois, N. Van Nhung, and J. Pierre, "Propriétés magnétiques des composés équiatomiques terres rares rhodium TRh $(\mathrm{T}=\mathrm{Tb}, \mathrm{Dy}, \mathrm{Ho}$, Er)," Phys. Status Solidi B 49, 161 (1972).

${ }^{392}$ R. Chamard-Bois, N. van Nhung, J. Yakinthos, and M. M. Wintenberger, "Structures magnetiques des composes equiatomiques terres raresrhodium T Rh(T=Dy, Ho, Er)," Solid State Commun. 10, 685-689 (1972).

${ }^{393}$ G. Scheunert, T. A. Ambrose, W. R. Hendren, A. A. Lapicki, P. Egan, R. Hardeman, M. Gubbins, and R. M. Bowman, "Ferromagnetism in DyRh and DyRhX (X=Fe, Ni, Co, Gd) thin films," J. Phys. D: Appl. Phys. 47(48), 485002 (2014).

${ }^{394}$ G. Z. Gadioli, F. P. Rouxinol, R. V. Gelamo, A. O. dos Santos, L. P. Cardoso, and M. A. Bica de Moraes, "Magnetism in Gd-W films," J. Appl. Phys. 103, 093916 (2008).

${ }^{395}$ Z. S. Shan, S. Nafis, K. D. Aylesworth, and D. J. Sellmyer, "Magnetic properties, anisotropy, and microstructure of sputtered rare earth iron multilayers," J. Appl. Phys. 63, 3218 (1988).

${ }^{396}$ A. Barth, "Magnetic and Structural Properties of the Gd/Ni-Bilayer System," PhD Dissertation (University of Konstanz, Konstanz, 2007); available at http://kops.uni-konstanz.de/handle/123456789/5175.

${ }^{397}$ R. Ranchal, C. Aroca, and E. López, "Domain walls and exchangeinteraction in Permalloy/Gd films," New J. Phys. 10, 013013 (2008).

${ }^{398}$ M. Romera, M. Munoz, M. Maicas, J. M. Michalik, J. M. de Teresa, C. Magen, and J. L. Prieto, "Enhanced exchange and reduced magnetization of Gd in an Fe/Gd/Fe trilayer," Phys. Rev. B 84, 094456 (2011). 
${ }^{399}$ S. Tsunashima, "Magneto-optical recording," J. Phys. D: Appl. Phys. 34, R87 (2001).

${ }^{400}$ Y. Uchihara, K. Tanase, Y. Suzuki, and K. Torazawa, "Thickness dependence of magnetic properties in GdDy/FeCo film," IEEE Trans. Magn. 29(6), 3784 (1993).

${ }^{401}$ B. F. DeSavage, R. M. Bozorth, F. E. Wang, and E. R. Callen, "Magnetization of the rare-earth manganese compounds $\mathrm{R}_{6} \mathrm{Mn}_{23}$," J. Appl. Phys. 36(3), 992 (1965).

${ }^{402}$ W. M. Hubbard, E. Adams, and J. V. Gilfrich, "Magnetic moments of alloys of gadolinium with some of the transition elements," J. Appl. Phys. 31(5), 368S (1960).

${ }^{403} \mathrm{P}$. Lemoine, Contribution à l'étude des propriétés structurales et magnétiques de composés intermétalliques isotypes de CeScSi et Th6Mn23. Physique, Géosciences, Chimie, Mécanique, Nancy Université, Université Henri Poincaré, Vandoeuvre-lès-Nancy, 2011; available at http://www.theses.fr/2011NAN10077/document.

${ }^{404} \mathrm{H}$. R. Kirchmayr and W. Steiner, "Magnetic order of the compound series $\mathrm{RE}_{6}\left(\mathrm{Mn}_{\mathrm{x}} \mathrm{Fe}_{1-\mathrm{x}}\right)_{23}(\mathrm{RE}=\mathrm{Y}, \mathrm{Gd})$, J. Phys. Colloq. 32(C1), 665-667 (1971).

${ }^{405}$ I. A. Campbell, "Indirect exchange for rare earths in metals," J. Phys. F: Met. Phys. 2, L47 (1972).

${ }^{406} \mathrm{~L}$. Zhang, "Unusual magnetic behavior of some rare-earth and manganese compounds," Ph.D. thesis, Universiteit van Amsterdam, Amsterdam, Holland, 2005; available at http://dare.uva.nl/record/1/238302.

${ }^{407}$ A. Delapalme, J. Déportes, R. Lemaire, K. Hardman, and W. J. James, "Magnetic interactions in $\mathrm{R}_{6} \mathrm{Mn}_{23}$ rare earth intermetallics," J. Appl. Phys. 50, 1987-1989 (1979).

${ }^{408}$ I. Zoto and G. Mankey, "Magnetic properties of coupled $\mathrm{Gd} / \mathrm{Pd} / \mathrm{Ni}$ thin films," Bull. Am. Phys. Soc. R23, 7 (2006); available at http://meetings. aps.org/Meeting/MAR06/Session/R23.7.

${ }^{409}$ B. Sanyal, C. Antoniak, T. Burkert, B. Krumme, A. Warland, F. Stromberg, C. Praetorius, K. Fauth, H. Wende, and O. Eriksson, "Forcing ferromagnetic coupling between rare-earth-metal and $3 \mathrm{~d}$ ferromagnetic films," Phys. Rev. Lett. 104, 156402 (2010).

${ }^{410}$ F. Stromberg, C. Antoniak, U. von Hörsten, W. Keune, B. Sanyal, O. Eriksson, and H. Wende, "Textured growth of the high moment material $\operatorname{Gd}(0-0-0-1) / \operatorname{Cr}(0-0-1) / \mathrm{Fe}(0-0-1)$, , J. Phys. D: Appl. Phys. 44, 265004 (2011).

${ }^{411}$ A. J. Freeman and O. G. Heinonen, "High moment soft magnetic materials for advanced writer applications via a quantum engineering approach" (unpublished).

${ }^{412}$ S. Nagase, H. Watanabe, and T. Shinohara, "Magnetic properties of the system $\mathrm{Fe}_{2} \mathrm{P}-\mathrm{Mn}_{2} \mathrm{P}$,” J. Phys. Soc. Jpn. 34, 908-910 (1973).

${ }^{413} \mathrm{~K}$. Motizuki, H. Ido, T. Itoh, and M. Morifuji, "Overview of magnetic properties of NiAs-type (MnP-type) and $\mathrm{Cu}_{2} \mathrm{Sb}$-type compounds," Springer Ser. Mater. Sci. 131, 11 (2010).

${ }^{414} \mathrm{G}$. Busch, "Magnetic properties of rare-earth compounds," J. Appl. Phys. 38(3), 1386 (1967).

${ }^{415}$ A. B. Drovosekov, N. M. Kreines, A. O. Savitsky, E. A. Kravtsov, D. V. Blagodatkov, M. V. Ryabukhina, M. A. Milyaev, V. V. Ustinov, E. M. Pashaev, I. A. Subbotin, and G. V. Prutskov, "Interlayer coupling in $\mathrm{Fe} / \mathrm{Cr} / \mathrm{Gd}$ multilayer structures,” J. Exp. Theor. Phys. 120, 1041-1054 (2015).

${ }^{416} \mathrm{C}$. Turtur and G. Bayreuther, "Magnetic moments in ultrathin $\mathrm{Cr}$ films on Fe (100)," Phys. Rev. Lett. 72(10), 1557 (1994).

${ }^{417}$ D. Knabben, T. Koop, H. A. Duerr, F. U. Hillebrecht, and G. van der Laan, "Cr magnetic moments in Fe-Cr layered structures," J. Electron Spectrosc. Relat. Phenom. 86, 201 (1997).

${ }^{418}$ N. S. Yartseva, S. V. Yartsev, J.-C. Parlebas, and C. Demangeat, "Role of noncollinearity of $\mathrm{Cr}$ magnetic moment distribution in $\mathrm{Fe} / \mathrm{Cr}$ superlattices," Eur. Phys. J. B 86, 167 (2013).

${ }^{419}$ Y. Li, C. Polaczyk, F. Mezei, and D. Riegel, "Magnetic properties of Gd/ $\mathrm{Fe}$ and Fe/Gd bilayer systems," Phys. B 234-236, 489 (1997).

${ }^{420} \mathrm{M}$. Loewenhaupt and W. Hahn, "Spin structures of Fe/Gd and Fe/Cr multilayers determined by polarized neutron reflectometry," J. Magn. Magn. Mater. 121, 173 (1993).

${ }^{421}$ A. B. Shick and W. E. Picket, "Electron correlation effects and magnetic ordering at the $\mathrm{Gd}(0001)$ surface," Phys. Rev. B 61(14), R9213(R) (2000).

${ }^{422}$ R. H. Kodama, "Magnetic nanoparticles," J. Magn. Magn. Mater. 200, 359 (1999).

${ }^{423} \mathrm{O}$. Sipr, M. Kosuth, and H. Ebert, "Magnetic structure of free iron clusters compared to iron crystal surfaces," Phys. Rev. B 70, 174423 (2004).
${ }^{424}$ S. Polesya, O. Sipr, S. Bornemann, J. Minar, and H. Ebert, "Magnetic properties of free Fe clusters at finite temperatures from first principles," Europhys. Lett. 74(6), 1074 (2006).

${ }^{425}$ J. Kortus, T. Baruah, M. R. Pederson, C. Ashman, and S. N. Khanna, "Magnetic moment and anisotropy in $\mathrm{Fe}_{\mathrm{n}} \mathrm{Co}_{\mathrm{m}}$ clusters," Appl. Phys. Lett. 80, 4193 (2002).

${ }^{426}$ I. M. L. Billas, J. A. Becker, A. Chatelain, and W. A. de Heer, "Magnetic moments of iron clusters with 25 to 700 atoms and their dependence on temperature," Phys. Rev. Lett. 71(24), 4067 (1993).

${ }^{427}$ K. W. Edmonds, C. Binns, S. H. Baker, S. C. Thornton, C. Norris, J. B. Goedkoop, M. Finazzi, and N. B. Brookes, "Doubling of the orbital magnetic moment in nanoscale Fe clusters," Phys. Rev. B 60, 472-476 (1999).

${ }^{428}$ S. H. Baker, C. Binns, K. W. Edmonds, M. J. Maher, S. C. Thornton, S. Louch, and S. S. Dhesi, "Enhancements in magnetic moments of exposed and Co-coated Fe nanoclusters as a function of cluster size," J. Magn. Magn. Mater. 247, 19-25 (2002).

${ }^{429}$ C. Binns, S. Louch, S. Baker, K. Edmonds, M. J. Maher, and S. C. Thornton, "High-moment films produced by assembling nanoclusters," IEEE Trans. Magn. 38, 141-145 (2002).

${ }^{430}$ S. Polesya, O. Sipr, S. Bornemann, J. Minar, and H. Ebert, "Magnetic properties of free Fe clusters at finite temperatures from first principles," Europhysics Letters 74(6), 1074 (2006).

${ }^{431}$ S. H. Baker, M. Roy, S. C. Thornton, and C. Binns, "Realizing high magnetic moments in fcc Fe nanoparticles through atomic structure stretch," J. Phys.: Condens. Matter 24(17), 176001 (2012).

${ }^{432}$ W. Gong, H. Li, Z. Zhao, and J. Chen, "Ultrafine particles of $\mathrm{Fe}, \mathrm{Co}$, and Ni ferromagnetic metals," J. Appl. Phys. 69, 5119 (1991).

${ }^{433}$ J. Bai and J.-P. Wang, "High-magnetic-moment core-shell-type FeCoAu/Ag nanoparticles," Appl. Phys. Lett. 87, 152502 (2005).

${ }^{434}$ W. S. Seo, J. H. Lee, X. Sun, Y. Suzuki, D. Mann, Z. Liu, M. Terashima, P. C. Yang, M. V. McConnell, D. G. Nishimura, and H. Dai, "FeCo/graphitic-shell nanocrystals as advanced magnetic-resonance-imaging and near-infrared agents," Nat. Mater. 5, 971 (2006).

${ }^{435}$ G.-M. Zhao, J. Wang, Y. Ren, and P. Beeli, "Giant enhancement and anomalous thermal hysteresis of saturation moment in magnetic nanoparticles embedded in multiwalled carbon nanotubes," Nano Lett. 13(6), 2993 (2013).

${ }^{436}$ A. J. Freeman and R. Wu, "Magnetic moment enhancement at transition metal and rare-earth metal surfaces," Prog. Theor. Phys. Suppl. 106, 397 (1991).

${ }^{437}$ I. Aruna, B. R. Mehta, L. K. Malhotra, and S. M. Shivaprasad, "Stability and hydrogenation of "Bare" gadolinium nanoparticles," Adv. Funct. Mater. 15, 131 (2005).

${ }^{438}$ J. P. Bucher and L. A. Bloomfield, "Magnetism of free transition metal and rare earth clusters," Int. J. Mod. Phys. B 7(4), 1079 (1993).

${ }^{439}$ J. Bansmann, S. H. Baker, C. Binns, J. A. Blackman, J.-P. Bucher, J. Dorantes-Davila, V. Dupius, L. Favre, D. Kechrakos, A. Kleibert, K.-H. Meiwes-Broer, G. M. Pastor, A. Perez, O. Toulemonde, K. N. Trohidou, J. Tuaillon, and Y. Xie, "Magnetic and structural properties of isolated and assembled clusters," Surf. Sci. Rep. 56, 189 (2005).

${ }^{440}$ M. Yue, J. X. Zhang, H. Zeng, and K. J. Wang, "Preparation, microstructure, and magnetic properties of bulk nanocrystalline Gd metal," Appl. Phys. Lett. 89, 232504 (2006).

${ }^{441}$ M. J. O'Shea and P. Perera, "Influence of nanostructure (layers and particles) on the magnetism of rare-earth materials," J. Appl. Phys. 85, 4322 (1999).

${ }^{442}$ C.-J. Hsu, S. V. Prikhodko, C.-Y. Wang, L.-J. Chen, and G. P. Carman, "Magnetic anisotropy in nanostructured gadolinium," J. Appl. Phys. 111, 053916 (2012).

${ }^{443}$ D. Johnson, P. Perera, and M. J. O'Shea, "Finite size effects in nanoscale Tb particles," J. Appl. Phys. 79(8), 5299 (1996).

${ }^{444}$ C. Suryanarayana, D. Mukhopadhyay, S. N. Patankar, and F. H. Froes, "Grain size effects in nanocrystalline materials," J. Mater. Res. 7(8), 2114 (1992).

${ }^{445}$ G. Vallejo-Fernandez, L. E. Fernandez-Outon, and K. O'Grady, "Antiferromagnetic grain volume effects in metallic polycrystalline exchange bias systems," J. Phys. D: Appl. Phys. 41, 112001 (2008).

${ }^{446} \mathrm{G}$. Herzer, "Nanocrystalline soft magnetic alloys," in Handbook of Magnetic Materials, edited by K. H. J. Buschow (Elsevier, 1997), Vol. 10 , pp. 415-462.

${ }^{447}$ A. D. Moral and E. W. Lee, "The reversible susceptibilities of dysprosium and terbium,” J. Phys. F: Met. Phys. 4, 280 (1974). 
${ }^{448}$ M. Daniil, K. E. Knipling, H. M. Fonda, and M. A. Willard, "Nonequilibrium materials design: A case study of nanostructured soft magnets for cryogenic applications," New J. Phys. 16, 055016 (2014).

${ }^{449}$ W. Jayasekara, J. Bain, and M. Kryder, "High frequency initial permeability of NiFe and FeAIN," IEEE Trans. Magn. 34, 1438-1440 (1998).

${ }^{450}$ D. Y. Park, B. Y. Yoo, S. Kelcher, and N. Myung, "Electrodeposition of low-stress high magnetic moment Fe-rich FeCoNi thin films," Electrochim. Acta 51, 2523-2530 (2006).

${ }^{451}$ H. Okumura, D. J. Twisselmann, R. D. McMichael, M. Q. Huang, Y. N. Hsu, D. E. Laughlin, and M. E. McHenry, "Magnetic and structural characterization and ferromagnetic resonance study of thin film HITPERM soft magnetic materials for data storage applications," J. Appl. Phys. 93, 6528-6530 (2003).

${ }^{452}$ K. E. Knipling, M. Daniil, and M. A. Willard, "Nanocrystalline $\mathrm{Fe}_{88-\mathrm{x}} \mathrm{Co}_{\mathrm{x}} \mathrm{Ni}_{\mathrm{x}} \mathrm{Zr}_{7} \mathrm{~B}_{4} \mathrm{Cu}_{1}$ alloys: Soft magnets for vehicle electrification technologies (invited)," J. Appl. Phys. 117, 172611 (2015).
${ }^{453}$ H. Matsuyama, H. Eguchi, and H. Karamon, "The high-resistive soft magnetic amorphous films consisting of cobalt, iron, boron, silicon, and oxygen, utilized for video head devices," J. Appl. Phys. 67, 5123-5125 (1990).

${ }^{454}$ S. Wang, F. Liu, K. Maranowski, and M. Kryder, "Fabrication and performance of high moment laminated FeAIN thin film inductive recording heads," IEEE Trans. Magn. 30, 281-286 (1994).

${ }^{455}$ C. Cheng, R. Davies, N. Sturcken, K. Shepard, and W. E. Bailey, "Optimization of ultra-soft $\mathrm{CoZrTa} / \mathrm{SiO}_{2} / \mathrm{CoZrTa}$ trilayer elements for integrated inductor structures," J. Appl. Phys. 113, 17A343 (2013).

${ }^{456}$ S. Bie, J. Jiang, G. Du, Q. Ma, L. Yuan, Y. Di, Z. Feng, and H. He, "Tb doped CoNbZr soft magnetic films with high anisotropy field for applications in GHz frequency region,” J. Alloys Compd. 463, 471-474 (2008).

${ }^{457}$ A. Hirohata, H. Sukegawa, H. Yanagihara, I. Zutic, T. Seki, S. Mizukami, and R. Swaminathan, "Roadmap for emerging magnetic materials for spintronic device applications," IEEE Trans. Magn. 51(10), 1 (2015). 